\title{
Metastability of breather modes of time-dependent potentials
}

\author{
P D Miller†, A Sofferł and M I Weinstein§ \\ $\dagger$ Department of Mathematics and Statistics, Monash University, Clayton, Victoria 3800, \\ Australia \\ and \\ School of Mathematics, Institute for Advanced Study, Olden Lane, Princeton, NJ 08540, USA \\ \$ Department of Mathematics, Hill Center, Rutgers University, 110 Frelinghuysen Road, \\ Piscataway, NJ 08854-8019, USA \\ § Mathematical Sciences Research, Bell Labs 2C-358, Lucent Technologies, 600 Mountain \\ Avenue, Murray Hill, NJ 07974, USA \\ and \\ Department of Mathematics, University of Michigan, East Hall, 525 East University Avenue, \\ Ann Arbor, MI 48109-1109, USA \\ E-mail: millerpd@mail.maths.monash.edu.au, soffer@math.rutgers.edu and \\ miw@research.bell-labs.com
}

Received 27 August 1999

Recommended by L Bunimovich

\begin{abstract}
We study the solutions of linear Schrödinger equations in which the potential energy is a periodic function of time and is sufficiently localized in space. We consider the potential to be close to one that is time periodic and yet explicitly solvable. A large family of such potentials has been constructed and the corresponding Schrödinger equation solved by Miller and Akhmediev. Exact bound states, or breather modes, exist in the unperturbed problem and are found to be generically metastable in the presence of small periodic perturbations. Thus, these states are long-lived but eventually decay. On a time scale of order $\epsilon^{-2}$, where $\epsilon$ is a measure of the perturbation size, the decay is exponential, with a rate of decay given by an analogue of Fermi's golden rule. For times of order $\epsilon^{-1}$ the breather modes are frequency shifted. This behaviour is derived first by classical multiple-scale expansions, and then in certain circumstances we are able to apply the rigorous theory developed by Soffer and Weinstein and extended by Kirr and Weinstein to justify the expansions and also provide longer-time asymptotics that indicate eventual dispersive decay of the bound states with behaviour that is algebraic in time. As an application, we use our techniques to study the frequency dependence of the guidance properties of certain optical waveguides. We supplement our results with numerical experiments.
\end{abstract}

AMS classification scheme numbers: 37K55, 35C20, 35C15, 35Q55, 81Q05, 81Q15

\section{Introduction and overview}

We are interested in the initial-value problem for the linear Schrödinger equation in one space dimension

$$
\mathrm{i} \partial_{t} f=\left(-\frac{1}{2} \partial_{x}^{2}+V(x, t)\right) f \equiv \mathcal{H}(t) f .
$$

Here, $f$ is a complex-valued function of $x \in \mathbb{R}$ and $t \in \mathbb{R}$. We assume that $V(x, t)$ is a smooth real-valued potential energy function which is sufficiently localized in space (for example, of Schwartz class). In our specific applications, $V(x, t)$ will be taken to be a periodic function 
of $t$. However, the techniques we use can be adapted to a more general time dependence [15]. Note that (1.1) is a non-autonomous Hamiltonian system

$$
\mathrm{i} \partial_{t} f=\frac{\delta h}{\delta f^{*}}\left[f, f^{*}, t\right]
$$

where $h$ denotes the Hamiltonian energy

$$
h\left[f, f^{*}, t\right]=\int\left(\frac{1}{2}\left|\partial_{x} f\right|^{2}+V(x, t)|f|^{2}\right) \mathrm{d} x .
$$

If $V(x, t)$ is not independent of $t, h$ is not a conserved integral of the flow. On the other hand, since the potential $V$ is real-valued, the flow defined by $(1.1)$ is always unitary in $L^{2}(\mathbb{R})$, i.e.

$$
\int|f(x, t)|^{2} \mathrm{~d} x=\int|f(x, 0)|^{2} \mathrm{~d} x \quad t \in \mathbb{R} .
$$

In applications, it is often natural to decompose $V(x, t)$ as

$$
V(x, t)=V_{0}(x, t)+W(x, t)
$$

where $V_{0}(x, t)$ denotes an unperturbed potential, and $W(x, t) \doteq V(x, t)-V_{0}(x, t)$, denotes a small perturbation. Thus,

$$
\mathcal{H}(t) \equiv \mathcal{H}_{0}(t)+\mathcal{W}(t)
$$

and (1.1) can be rewritten as

$$
\mathrm{i} \partial_{t} f=\left(\mathcal{H}_{0}(t)+\mathcal{W}(t)\right) f .
$$

Here, we have denoted the multiplication operator $f \mapsto W(x, t) f$ by $\mathcal{W}(t)$. The choice of $V_{0}(x, t)$ is often dictated by some a priori knowledge of the solutions of the unperturbed system

$$
\mathrm{i} \partial_{t} f=\mathcal{H}_{0}(t) f .
$$

A problem of importance is then to contrast the detailed dynamics of solutions to (1.6) with those of the unperturbed system (1.7). In particular, if (1.7) has bound state solutions (breather modes, or solutions having finite energy and not decaying as $|t| \rightarrow \infty$ ) do they persist in the perturbed dynamical system (1.6)?

The simplest variant of this problem is the case where the unperturbed part is stationary, i.e. $V_{0}(x, t)=V_{0}(x)$. Suppose the operator $\mathcal{H}_{0}$ has an $L^{2}$ eigenfunction. The unitary evolution of the spatially localized eigenfunction is time-periodic and represents a bound state solution of the unperturbed Schrödinger equation (1.7). The perturbed model (in this and in the more general case when $\mathcal{H}_{0}$ has multiple discrete eigenvalues) is related to the problem of ionization of an atom by a time-dependent electromagnetic field $[10,17]$ and the problem of describing the effects of weak inhomogeneities on the propagation of continuous waves in optical fibres [20]. Using a time-dependent method developed in the context of (a) quantum resonances and the perturbation theory of embedded eigenvalues in the continuous spectrum [26] and (b) resonances and radiation damping of bound nonlinear wave equations [27], Soffer and Weinstein studied the metastability of such states [28]. Generalizations of this theory for handling multi-frequency perturbations [15] and the interference of multiple bound states in the unperturbed problem [16] have been explored by Kirr and Weinstein. Based on the observation that the mechanism for instability of the bound state is coupling of the bound state to the continuous spectral modes, the analysis was carried out at the level of the coupled equations for the bound state and dispersive components of the solution. Under general hypotheses on 
the unperturbed Hamiltonian (local energy decay estimates on the unitary propagator $\mathrm{e}^{-\mathrm{i} t \mathcal{H}_{0}}$ ) this equivalent dynamical system was studied and it was shown that a bound state is generically unstable but long-lived. The lifetime is given by a formula analogous to Fermi's golden rule [28].

In this paper we consider the case where the unperturbed Hamiltonian is genuinely time dependent. A physical application of the theory we develop, in the context of frequency detuning in periodically modulated optical waveguides [2,3], will be presented in section 5 . Let $\mathcal{H}_{0}(t)=-\frac{1}{2} \partial_{x}^{2}+V_{0}(x, t)$, where $V_{0}(x, t)$ is smooth, periodic in $t$ with the same period for each $x$ and of sufficiently rapid decay for large $x$ for each $t \in \mathbb{R}$. The particular choices of $V_{0}(x, t)$ we consider in this paper belong to a large family of very special, so-called separable, time-dependent potentials, $V_{0}(x, t), x \in \mathbb{R}$, studied by Miller and Akhmediev [22]. The separable potential $V_{0}(x, t)$ can be chosen to be time periodic, in which case the unperturbed problem supports exact bound states (breather modes) and the initial-value problem for (1.7) can be solved exactly. That is, a complete set of eigenmodes and generalized eigenmodes can be displayed explicitly with respect to which the dynamics of (1.7) is diagonal. This class of potentials is intimately connected with the soliton theory of completely integrable multicomponent cubic nonlinear Schrödinger equations [9, 19].

The existence of such exact breather modes in the unperturbed time-periodic problem is quite remarkable and we believe that this is a highly non-generic phenomenon $\dagger$. Indeed, from a general dynamical systems perspective, equation (1.7) with such a choice of $V_{0}(x, t)$, may be viewed as a parametrically forced wave equation (here we are actually considering the timeperiodic function $V_{0}(x, t)$ itself to be the sum of a time-independent part and a time-periodic modulation). One therefore expects that the presence of resonances will perturb the Floquet multipliers (corresponding to bound states) off the unit circle as in the elementary example of Mathieu's equation [1]. The persistence of breather solutions under the time-periodic perturbation would imply the non-departure of a Floquet multiplier from the unit circle to all orders in the size of the perturbation. The fact that infinitely many such conditions hold for these special separable potentials is no doubt linked to the infinite sequence of symmetries and time invariants enjoyed by the completely integrable nonlinear flow that underpins the construction of the separable potentials (see appendix A for more details). Of course, this is only a heuristic picture. In fact, the perturbation theory of the Floquet multipliers is complicated by the fact that they are embedded in the continuous spectrum which covers the unit circle. However, spectral deformation methods have been developed for some classes of models that could well be adapted here. Relevant technical details can be found in $[6,11] \$$.

We want to make our motivation for pursuing deformations of these admittedly rather special periodic potentials very clear. First of all, the problem is relevant to the analysis of optical waveguides. In the paraxial approximation, the slowly varying envelope of a highly oscillatory electric field in a dielectric medium with inhomogeneous dielectric properties (refractive index) satisfies an equation of the form (1.1). Here, $t$ denotes the

$\dagger$ The scarcity of breather solutions of nonlinear wave equations defined on a spatial continuum of infinite extent has been explored extensively in the setting of perturbations of the completely integrable sine-Gordon equation; see, for example, $[4,5,8,14]$. The connection with linear non-autonomous problems can be made by viewing a breather solution of a nonlinear dynamical problem as a bound state of a linear problem with a given (self-consistent) potential. $\ddagger$ For nonlinear wave equations defined on an infinite lattice (e.g. discrete sine-Gordon, discrete $\phi^{4}$ ), breather solutions can be constructed for sufficiently large lattice spacing; see, for example, [18]. The radiative decay of such discrete breathers, for sufficiently small lattice spacing, is expected to be governed by a mechanism of the kind studied in this paper (see also [27]). Related to this are results for the dynamics of kinks of discrete nonlinear wave equations, in which the techniques of this paper have been used to study the 'pinning' of discrete kinks on a lattice site. This pinning is marked by the slow radiative decay of spatially localized and time-periodic or quasiperiodic oscillations about a static kink [13]. 
longitudinal variable, the direction of propagation, and $x$ is the transverse spatial variable. For inhomogeneous index profiles corresponding to exactly solvable potentials, light of $a$ particular frequency propagates as a non-attenuating bound state mode in these waveguides. However, if the light frequency deviates from the 'integrable frequency', the propagating wave will be governed by the perturbed equation (1.6). Thus the question of whether such modes persist and if not what their lifetime is for the perturbed dynamics naturally arises. We will give more details about this problem in section 5 .

However, it is also true that the study of perturbed separable periodic potentials is important in general terms. Given an arbitrary time-periodic potential in the Schrödinger equation, one wants to study the corresponding dynamics using perturbation theory. In doing so, the first question one must address is that of finding a 'nearby' problem that can be solved exactly. We simply take the point of view that many periodic potentials will be closer to a separable periodic potential (in a sense that can be made precise) than to any time-independent potential.

In any case, with the explicit spectral theory associated with $V_{0}(x, t)$ in hand, our goal is to carry out a detailed analytical study of the coupled-mode dynamics induced by a timedependent perturbation $W(x, t)$. We establish the generic metastabilty of the exact bound states associated with separable periodic potentials $V_{0}(x, t)$ and obtain a detailed picture of the dynamics.

This paper is structured as follows. In section 2 we first review the construction of timedependent exactly solvable potentials [22] from a set of discrete data, and then show how the initial-value problem for such separable potentials can be solved explicitly. We then describe how properties of the separable potentials depend on the choice of the discrete data generating them. Next, we derive by projection onto an orthonormal basis the general coupled-mode equations which arise when a separable potential is perturbed by some arbitrary correction $W(x, t)$. This section will then conclude with a detailed derivation of the two-soliton timeperiodic potential and its associated explicit complete set of bound states and generalized eigenfunctions. More details about the separable potentials described in section 2 are given in appendix A.

In section 3, Floquet theory is then used to map the coupled-mode equations to a system associated with a time-dependent perturbation of an autonomous system, a situation analysed in detail in $[15,28]$. In section 4 , we then describe the dynamics of solutions of the coupledmode equations for even time-periodic perturbations $W(x, t)$ of a separable two-soliton even time-periodic potential $V_{0}(x, t)\left(V_{0}(x, t)\right.$ and $W(x, t)$ both share even parity in $x$ and have the same temporal period). In particular, we study the initial-value problem when the initial condition is a pure bound state of the unperturbed problem. First, we study the small-time behaviour of the coupled-mode equations (without requiring $W(x, t)$ to be small) and deduce that the bound state amplitude behaves as $1-C t^{2}$ for some constant $C$ and interpret this result in the context of the theory of ideal measurements in quantum mechanics (the 'watched pot' effect). We then assume the perturbation $W(x, t)$ to be small, of size $\epsilon$, and seek the behaviour of the bound state amplitude over intermediate times of order $\epsilon^{-1}$ and $\epsilon^{-2}$ using the classical method of multiple scales.

We show the existence of a perturbation-induced frequency shift of the breather mode evident on time scales of order $\epsilon^{-1}$ and exponential decay of the bound state mode amplitude on time scales of order $\epsilon^{-2}$. The condition for the decay constant to be non-zero is a direct analogue of 'Fermi's golden rule'.

Then, using the transformation to an autonomous system found in section 3, we show how the rigorous theory developed for multi-frequency perturbations of autonomous systems by Kirr and Weinstein [15] can be applied in some cases to justify the multiple-scales calculation, and to provide more detailed information about the infinite-time behaviour of the solution. 
This analysis completes the portrait of the dynamics, showing that the exponential decay is ultimately washed out in a sea of dispersive waves, at which point the decay becomes algebraic in time.

Having described the theory, in section 5 we consider an application of the analysis to a problem of frequency detuning in planar optical waveguides. Finally, in section 6 the prediction of an exponential decay constant $\Gamma$ for the bound state mode amplitude found in section 4 is compared to numerical simulations of the perturbed time-dependent Schrödinger equation.

A detailed description of the theory of separable potentials, at once summarizing for completeness and also further developing the results of [22], can be found in appendix A. In appendix B the reader will find the proofs of the decay estimates that we will use in section 4 in order to apply the results of [15].

\section{Regarding notation}

We will use the inner product

$$
\langle f(\cdot), g(\cdot)\rangle=\int_{-\infty}^{\infty} f(x)^{*} g(x) \mathrm{d} x
$$

on $L^{2}(\mathbb{R})$. Occasionally, the angled brackets will denote the inner product in more general Hilbert spaces. Linear operators will be denoted by calligraphic letters, vectors by arrows and matrices with boldface letters. We will often use the function defined by

$$
\langle x\rangle \doteq\left(1+x^{2}\right)^{1 / 2} .
$$

Complex conjugation will be denoted with stars, and time averages will be denoted with bars.

\section{Exactly solvable time-dependent potentials}

In this section we recall for our purposes a class of time-dependent potentials $V_{0}(x, t)$ related to $M$-soliton solutions of certain completely integrable nonlinear flows. Because of the intimate connection of these potentials to integrable systems, it is possible to explicitly derive the spectral representation associated with such potentials [22]. This section is divided into five parts. First, the direct construction of separable potentials from a set of discrete data $\mathcal{D}$ is outlined. Then, we show how the same discrete data $\mathcal{D}$ gives rise to formulae for a complete set of modes for the time-dependent Schrödinger equation corresponding to the separable potential $V_{0}(x, t)$ and how this basis is easily used to express the general solution of the initial-value problem. We then give a qualitative description of the kinds of functions $V_{0}(x, t)$ one can obtain from this procedure. As we ultimately want to consider perturbations of $V_{0}(x, t)$, we next show how to use the basis of solutions to the unperturbed problem to derive the coupled-mode equations which trivialize the unperturbed dynamics and lay bare the perturbative effects. Finally, we specialize to the case of an even periodic potential corresponding to a two-soliton solution of the cubic nonlinear Schrödinger equation. As one might anticipate, the evenness (in $x$ ) of the potential leads to some simplifications in the spectral representation.

\subsection{Separable time-dependent potentials}

Let us present the construction of the family of time-dependent potentials that we will consider in this paper, and describe their properties with respect to the linear Schrödinger equation. More details can be found in appendix A. 
Each potential we shall consider will be specified by a certain set of discrete data. Let $N$ and $M$ be independent natural numbers. A set of discrete data $\mathcal{D}$ consists of $M$ distinct complex numbers $\lambda_{1}, \ldots, \lambda_{M}$ in the upper half-plane, and $M$ vectors $\vec{g}^{(1)}, \ldots, \vec{g}^{(M)}$ in $\mathbb{C}^{N}$.

The discrete data $\mathcal{D}$ are used to build a potential function $V_{0}(x, t)$ in the following way. Introduce the scalar expression

$$
a(x, t, \lambda)=\left(\lambda^{M}+\sum_{p=0}^{M-1} \lambda^{p} a^{(p)}(x, t)\right) \mathrm{e}^{-2 \mathrm{i}\left(\lambda x+\lambda^{2} t\right)}
$$

and the $N$-component vector expression

$$
\vec{b}(x, t, \lambda)=\sum_{p=0}^{M-1} \lambda^{p} \vec{b}^{(p)}(x, t) .
$$

In these expressions, the coefficients $a^{(p)}(x, t)$ and $\vec{b}^{(p)}(x, t)$ are undetermined functions of $x$ and $t$. They will now be determined by the use of the discrete data $\mathcal{D}$. For $k=1, \ldots, M$, we insist that $a(x, t, \lambda)$ and $\vec{b}(x, t, \lambda)$ satisfy the relations

$$
\begin{aligned}
& a\left(x, t, \lambda_{k}\right)=\vec{g}^{(k) \dagger} \vec{b}\left(x, t, \lambda_{k}\right) \\
& \vec{b}\left(x, t, \lambda_{k}^{*}\right)=-a\left(x, t, \lambda_{k}^{*}\right) \vec{g}^{(k)} .
\end{aligned}
$$

These equations amount to a square linear inhomogeneous system of algebraic equations for the coefficient functions $a^{(p)}(x, t)$ and the components of $\vec{b}^{(p)}(x, t)$. We will soon illustrate this procedure with a concrete example. From the solution of this linear system, the potential function connected with the discrete data $\mathcal{D}$ is given in terms of the components of $\vec{b}^{(M-1)}(x, t)$ by

$$
V_{0}(x, t) \doteq-4 \sum_{n=1}^{N}\left|b_{n}^{(M-1)}(x, t)\right|^{2} .
$$

This function $V_{0}(x, t)$ is a genuinely time-dependent potential well. Furthermore, it can be shown that $V_{0}(x, t)$ is in the Schwartz space as a function of $x$, and its $L^{1}$ norm is constant in $t$. The latter follows from the fact that $V_{0}(x, t)$ can be viewed as the self-consistent nonlinear potential for an $N$-component cubic nonlinear Schrödinger equation, which conserves the sum of the $L^{2}$ norms of the $N$ field components, which are proportional to the $b_{n}^{(M-1)}(x, t)$ for $n=1, \ldots, N$.

\subsection{Solution of the linear Schrödinger equation with a separable potential}

Along with the potential function $V_{0}(x, t)$, this construction starting from the discrete data $\mathcal{D}$ also provides all of the solutions of the corresponding linear Schrödinger equation [22]. These are built from the function $a(x, t, \lambda)$ as follows. For all real $\lambda$, set

$$
\Psi_{\mathrm{d}}(x, t, \lambda) \doteq\left(\pi \prod_{k=1}^{M}\left|\lambda-\lambda_{k}\right|^{2}\right)^{-1 / 2} a(x, t, \lambda)
$$

and let the functions $\Psi_{\mathrm{b}, 1}(x, t), \ldots, \Psi_{\mathrm{b}, M}(x, t)$ be the result of applying the Gram-Schmidt procedure (in $L^{2}(\mathbb{R})$ with respect to $\left.x\right)$ to the functions $a\left(x, t, \lambda_{1}^{*}\right), \ldots, a\left(x, t, \lambda_{M}^{*}\right)$ at any fixed value of $t$. Then we have [22]: 
(a) Each function $\Psi_{\mathrm{d}}(x, t, \lambda)$ for $\lambda \in \mathbb{R}$ and each function $\Psi_{\mathrm{b}, k}(x, t)$ is a solution of the linear Schrödinger equation with the potential $V_{0}(x, t)$. The fact that the $L^{2}$ inner product is an invariant of the evolution shows that the functions $\Psi_{\mathrm{b}, k}(x, t)$ do not depend on the choice of the time $t$ at which they are obtained from the Gram-Schmidt process.

(b) For any fixed $t$, these functions form an orthonormal basis of $L^{2}(\mathbb{R})$.

These facts show us how to solve the initial-value problem for the linear Schrödinger equation for the potential $V_{0}(x, t)$. Namely, to find the solution of

$$
\mathrm{i} \partial_{t} f+\frac{1}{2} \partial_{x}^{2} f-V_{0}(x, t) f=0 \quad f(x, 0)=f_{0}(x) \in L^{2}(\mathbb{R})
$$

one simply projects the initial data onto the basis at $t=0$ by defining

$$
\hat{f}(\lambda) \doteq\left\langle\Psi_{\mathrm{d}}(\cdot, 0, \lambda), f_{0}(\cdot)\right\rangle \quad \hat{f}_{k} \doteq\left\langle\Psi_{\mathrm{b}, k}(\cdot, 0), f_{0}(\cdot)\right\rangle
$$

and then recovers the solution as a superposition of modes

$$
f(x, t)=\sum_{k=1}^{M} \hat{f_{k}} \Psi_{\mathrm{b}, k}(x, t)+\int_{-\infty}^{\infty} \hat{f}(\lambda) \Psi_{\mathrm{d}}(x, t, \lambda) \mathrm{d} \lambda .
$$

If the potential $V_{0}(x, t)$ is slightly perturbed, it may still be convenient to expand in this basis, but then the coefficients $\hat{f}(\lambda)$ and $\hat{f}_{1}, \ldots, \hat{f}_{M}$ will become time-dependent.

\subsection{Qualitative description of separable potentials}

Let us describe the types of potential functions $V_{0}(x, t)$ that can be obtained by this procedure. In the generic case when the real parts of the parameters $\lambda_{1}, \ldots, \lambda_{M}$ are all distinct, these potentials have the form of a collision among $M$ moving potential wells. That is, as $t \rightarrow \pm \infty$,

$$
V_{0}(x, t) \sim \sum_{k=1}^{M} V_{0}^{(k) \pm}(x, t)
$$

where the individual wells have the form

$$
V_{0}^{(k) \pm}(x, t)=-4 \rho_{k}^{2} \operatorname{sech}^{2}\left(2 \rho_{k}\left(x+2 \sigma_{k} t\right)-\delta_{k}^{ \pm}\right)
$$

where $\delta_{k}^{ \pm}$are constants that depend on the vectors $\vec{g}^{(1)}, \ldots, \vec{g}^{(M)}$, and where $\lambda_{k}=\sigma_{k}+\mathrm{i} \rho_{k}$. Considered in isolation, each well carries a single bound state. When the wells collide for finite $t$, the bound states can become mixed, and a state $f(x, t)$ that is bound in a single well as $t \rightarrow-\infty$ will have a component in each well as $t \rightarrow+\infty$. The associated scattering matrix can be computed exactly [21].

If some of the parameters $\lambda_{k}$ share the same real part $\sigma$, then the asymptotics of the potential $V_{0}(x, t)$ in the frame moving with velocity $-2 \sigma$ will no longer be stationary, but will be generally quasiperiodic. In particular, if all of the parameters $\lambda_{k}$ are purely imaginary, then the potential $V_{0}(x, t)$ will generally be a quasiperiodic function of the time $t$. This is clear because taking $\lambda_{k}=\mathrm{i} \rho_{k}$ with $\rho_{k}$ real and positive ensures that the only time dependence that enters into the computation of $V_{0}(x, t)$ is via the exponentials $\exp \left( \pm 2 \mathrm{i} \rho_{k}^{2} t\right)$. Such a potential is automatically quasiperiodic. We can further ensure that the potential function is strictly periodic by making the frequencies commensurate. This will be true $\dagger$ if the parameters $\rho_{k}$ have the form

$$
\rho_{k}=\sqrt{\frac{1}{2} n_{k} \Omega_{0}+\Delta}
$$

$\dagger$ For $M>2$. The potential is always periodic if $M=2$ and is stationary if $M=1$. 
where $\Omega_{0}$ is some fundamental frequency and $n_{k}$ are distinct integers. This choice ensures that the frequencies

$$
\omega_{j k} \doteq 2 \rho_{j}^{2}-2 \rho_{k}^{2}=\left(n_{j}-n_{k}\right) \Omega_{0}
$$

are all integer multiples of $\Omega_{0}$. Only the frequency differences $\omega_{j k}$ are important because the potential is given as a sum of absolute values (2.4). In fact, it can be seen from the form of the linear system (2.3) that

$$
b_{n}^{(p)}(x, t)=\mathrm{e}^{2 \mathrm{i}\left(\rho_{1}^{2}+\cdots+\rho_{M}^{2}\right) t} G_{n, p}\left(\left\{\mathrm{e}^{\mathrm{i} \omega_{j k} t}\right\}, x\right)
$$

where $G_{n, p}$ is, for each fixed $x$, a rational function of the exponentials $\exp \left(\mathrm{i} \omega_{j k} t\right)$. The sufficiency of the relations (2.11) to guarantee time periodicity of $V(x, t)$ with a fundamental frequency $\Omega_{0}$ is then clear from (2.4).

\subsection{Perturbed separable potentials and coupled-mode equations}

As we have already suggested, the explicit basis of exact solutions derived in the previous subsection forms a natural coordinate system in which to study perturbed problems. Let $W(x, t)$ be a correction to the potential energy, so that the equation becomes

$$
\mathrm{i} f_{t}=\left(-\frac{1}{2} \partial_{x}^{2}+V_{0}(x, t)\right) f+W(x, t) f=\mathcal{H}_{0}(t) f+\mathcal{W}(t) f .
$$

Here, $V_{0}(x, t)$ is a separable time-dependent potential built from the discrete data $\mathcal{D}=$ $\left\{\lambda_{1}, \ldots, \lambda_{M}, \vec{g}^{(1)}, \ldots, \vec{g}^{(M)}\right\}$. So we use completeness to express $f(x, t)$ for each fixed $t$ in terms of the basis of solutions of the unperturbed problem

$$
f(x, t)=\sum_{k=1} B_{\mathrm{b}, k}(t) \Psi_{\mathrm{b}, k}(x, t)+\int_{-\infty}^{\infty} B_{\mathrm{d}}(t, \lambda) \Psi_{\mathrm{d}}(x, t, \lambda) \mathrm{d} \lambda .
$$

In the absence of the perturbation $W(x, t)$, the mode amplitudes $B_{\mathrm{b}, k}$ and $B_{\mathrm{d}}(t, \lambda), \lambda \in \mathbb{R}$ are governed by the equations $\partial_{t} B_{\mathrm{b}, k}=0, \partial_{t} B_{\mathrm{d}}(t, \lambda)=0$. In the presence of a perturbation $W(x, t)$ coupled-mode equations can be derived by projecting (2.14) onto the basis elements $\Psi_{\mathrm{b}, k}(x, t)$ and $\Psi_{\mathrm{d}}(x, t, \lambda)$. This yields the system of coupled-mode equations

$$
\begin{aligned}
& \mathrm{i} \partial_{t} \vec{B}_{\mathrm{b}}(t)=M(t) \vec{B}_{\mathrm{b}}(t)+\int_{-\infty}^{\infty} B_{\mathrm{d}}(t, \lambda) \vec{N}(t, \lambda) \mathrm{d} \lambda \\
& \mathrm{i} \partial_{t} B_{\mathrm{d}}(t, \eta)=\vec{N}(t, \eta)^{\dagger} \vec{B}_{\mathrm{b}}(t)+\int_{-\infty}^{\infty} K(t, \eta, \lambda) B_{\mathrm{d}}(t, \lambda) \mathrm{d} \lambda
\end{aligned}
$$

for the coefficients of $f(x, t)$, where $\vec{B}_{\mathrm{b}}(t)$ is the vector of bound state amplitudes $B_{\mathrm{b}, k}(t)$, and where the matrix elements of the perturbation $W(x, t)$ are given explicitly by

$$
\begin{aligned}
& M_{k j}(t)=\left\langle\Psi_{\mathrm{b}, k}(\cdot, t), W(\cdot, t) \Psi_{\mathrm{b}, j}(\cdot, t)\right\rangle \\
& N_{k}(t, \lambda)=\left\langle\Psi_{\mathrm{b}, k}(\cdot, t), W(\cdot, t) \Psi_{\mathrm{d}}(\cdot, t, \lambda)\right\rangle \\
& K(t, \eta, \lambda)=\left\langle\Psi_{\mathrm{d}}(\cdot, t, \eta), W(\cdot, t) \Psi_{\mathrm{d}}(\cdot, t, \lambda)\right\rangle
\end{aligned}
$$

where $N_{k}(t, \lambda)$ are the components of the vector $\vec{N}(t, \lambda)$ and $M_{k j}(t)$ are the elements of the matrix $\boldsymbol{M}(t)$. In particular, it follows that the matrix $\boldsymbol{M}(t)$ is Hermitian and the scalar kernel $K(t, \eta, \lambda)$ is Hermitian symmetric because $W(x, t)$ is real. With the unperturbed problem exactly diagonalized in this way, this system is a useful starting point for perturbation theory. 


\subsection{Even two-soliton periodic potentials}

In this subsection, we illustrate the procedures described above in some detail with an example that is important in applications and that will guide the subsequent discussion. We consider the case $N=1$ and $M=2$, and accordingly introduce the expressions

$$
\begin{aligned}
& a(x, t, \lambda)=\left(\lambda^{2}+a^{(1)}(x, t) \lambda+a^{(0)}(x, t)\right) \mathrm{e}^{-2 \mathrm{i}\left(\lambda x+\lambda^{2} t\right)} \\
& b(x, t, \lambda)=b^{(1)}(x, t) \lambda+b^{(0)}(x, t) .
\end{aligned}
$$

Because $N=1$ these are both scalar expressions, and we have at the moment four complexvalued unknown functions, $a^{(0)}(x, t), a^{(1)}(x, t), b^{(0)}(x, t)$ and $b^{(1)}(x, t)$. To find these, we introduce the discrete data $\lambda_{1}, \lambda_{2}, g^{(1)}$ and $g^{(2)}$ (again, here the $g^{(k)}$ are complex scalars because $N=1)$. The linear equations (2.3) then become

$$
\begin{aligned}
& \left(\lambda_{1}^{2}+a^{(1)}(x, t) \lambda_{1}+a^{(0)}(x, t)\right) \mathrm{e}^{-2 \mathrm{i}\left(\lambda_{1} x+\lambda_{1}^{2} t\right)}=g^{(1) *}\left(b^{(1)}(x, t) \lambda_{1}+b^{(0)}(x, t)\right) \\
& \left(\lambda_{2}^{2}+a^{(1)}(x, t) \lambda_{2}+a^{(0)}(x, t)\right) \mathrm{e}^{-2 \mathrm{i}\left(\lambda_{2} x+\lambda_{2}^{2} t\right)}=g^{(2) *}\left(b^{(1)}(x, t) \lambda_{2}+b^{(0)}(x, t)\right)
\end{aligned}
$$

and

$$
\begin{aligned}
& b^{(1)}(x, t) \lambda_{1}^{*}+b^{(0)}(x, t)=-g^{(1)}\left(\lambda_{1}^{* 2}+a^{(1)}(x, t) \lambda_{1}^{*}+a^{(0)}(x, t)\right) \mathrm{e}^{-2 \mathrm{i}\left(\lambda_{1}^{*} x+\lambda_{1}^{* 2} t\right)} \\
& b^{(1)}(x, t) \lambda_{2}^{*}+b^{(0)}(x, t)=-g^{(2)}\left(\lambda_{2}^{* 2}+a^{(1)}(x, t) \lambda_{2}^{*}+a^{(0)}(x, t)\right) \mathrm{e}^{-2 \mathrm{i}\left(\lambda_{2}^{*} x+\lambda_{2}^{* 2} t\right)} .
\end{aligned}
$$

Given the discrete data $\mathcal{D}$, one can solve these equations for $a^{(0)}(x, t), a^{(1)}(x, t), b^{(0)}(x, t)$ and $b^{(1)}(x, t)$, say by Cramer's rule, and thus obtain explicit expressions in terms of exponential functions.

Specializing to the case of $\lambda_{1}=\mathrm{i} \rho_{1}, \lambda_{2}=\mathrm{i} \rho_{2}$ (we assume without loss of generality that $\rho_{2}>\rho_{1}$ ), we obtain a time-periodic potential function, since the parameters $\lambda_{k}$ are pure imaginary and then the commensurability condition is automatically satisfied for $M=2$. Furthermore, choosing $g^{(1)}=\mathrm{e}^{\mathrm{i} \theta_{1}}$ and $g^{(2)}=\mathrm{e}^{\mathrm{i} \theta_{2}}$ ensures that the potential function is even in $x$. Indeed, we then find that with $s=\rho_{2}+\rho_{1}$ and $d=\rho_{2}-\rho_{1}$,

$b^{(1)}(x, t)=2 s d \frac{\rho_{1} \cosh \left(2 \rho_{2} x\right) \mathrm{e}^{2 \mathrm{i} \rho_{1}^{2} t+\mathrm{i} \theta_{1}}-\rho_{2} \cosh \left(2 \rho_{1} x\right) \mathrm{e}^{2 \mathrm{i} \rho_{2}^{2} t+\mathrm{i} \theta_{2}}}{d^{2} \cosh (2 s x)+s^{2} \cosh (2 d x)-4 \rho_{1} \rho_{2} \cos \left(2 s d t+\theta_{2}-\theta_{1}\right)}$.

The potential function is then given by

$$
V_{0}(x, t)=-4\left|b^{(1)}(x, t)\right|^{2}
$$

which is easily seen to be periodic in $t$ with period $L=\pi /(s d)$, and an even function of $x$. The shapes of these time-periodic potential wells are shown in figures 1 and 2 for $\theta_{1}=\theta_{2}=0$ and two different choices of the parameters $\rho_{1}$ and $\rho_{2}$. From the solution of the same linear system, we also find

$a^{(0)}(x, t)=\rho_{1} \rho_{2} \frac{\left(\rho_{1}+\rho_{2}\right)^{2} S_{1} S_{2}+\rho_{1}^{2} \mathrm{e}^{-\mathrm{i} \omega t-\mathrm{i}\left(\theta_{2}-\theta_{1}\right)}+\rho_{2}^{2} \mathrm{e}^{\mathrm{i} \omega t+\mathrm{i}\left(\theta_{2}-\theta_{1}\right)}-2 \rho_{1} \rho_{2} C}{2 \rho_{1} \rho_{2} \cos \left(\omega t+\left(\theta_{2}-\theta_{1}\right)\right)-\left(\rho_{1}^{2}+\rho_{2}^{2}\right) C+\left(\rho_{1}+\rho_{2}\right)^{2} S_{1} S_{2}}$

and

$a^{(1)}(x, t)=\mathrm{i} \frac{\left(\rho_{1}^{2}-\rho_{2}^{2}\right) \rho_{1} C_{2} S_{1}+\left(\rho_{2}^{2}-\rho_{1}^{2}\right) \rho_{2} C_{1} S_{2}}{2 \rho_{1} \rho_{2} \cos \left(\omega t+\left(\theta_{2}-\theta_{1}\right)\right)-\left(\rho_{1}^{2}+\rho_{2}^{2}\right) C+\left(\rho_{1}+\rho_{2}\right)^{2} S_{1} S_{2}}$

where $S_{k} \doteq \sinh \left(2 \rho_{k} x\right), C_{k} \doteq \cosh \left(2 \rho_{k} x\right)$ and $C \doteq \cosh \left(2\left(\rho_{1}+\rho_{2}\right) x\right)$, and where the frequency is $\omega=2 \pi / L=2 s d$. Note that $a^{(0)}(x, t)$ is an even function of $x$, while $a^{(1)}(x, t)$ is odd. We may then write the mode function $a(x, t, \lambda)$ in the form

$$
\begin{aligned}
a(x, t, \lambda)=( & \left.\left(\lambda^{2}+a^{(0)}(x, t)\right) \cosh (-2 \mathrm{i} \lambda x)+\lambda a^{(1)}(x, t) \sinh (-2 \mathrm{i} \lambda x)\right) \mathrm{e}^{-2 \mathrm{i} \lambda^{2} t} \\
& +\left(\left(\lambda^{2}+a^{(0)}(x, t)\right) \sinh (-2 \mathrm{i} \lambda x)+\lambda a^{(1)}(x, t) \cosh (-2 \mathrm{i} \lambda x)\right) \mathrm{e}^{-2 \mathrm{i} \lambda^{2} t}
\end{aligned}
$$




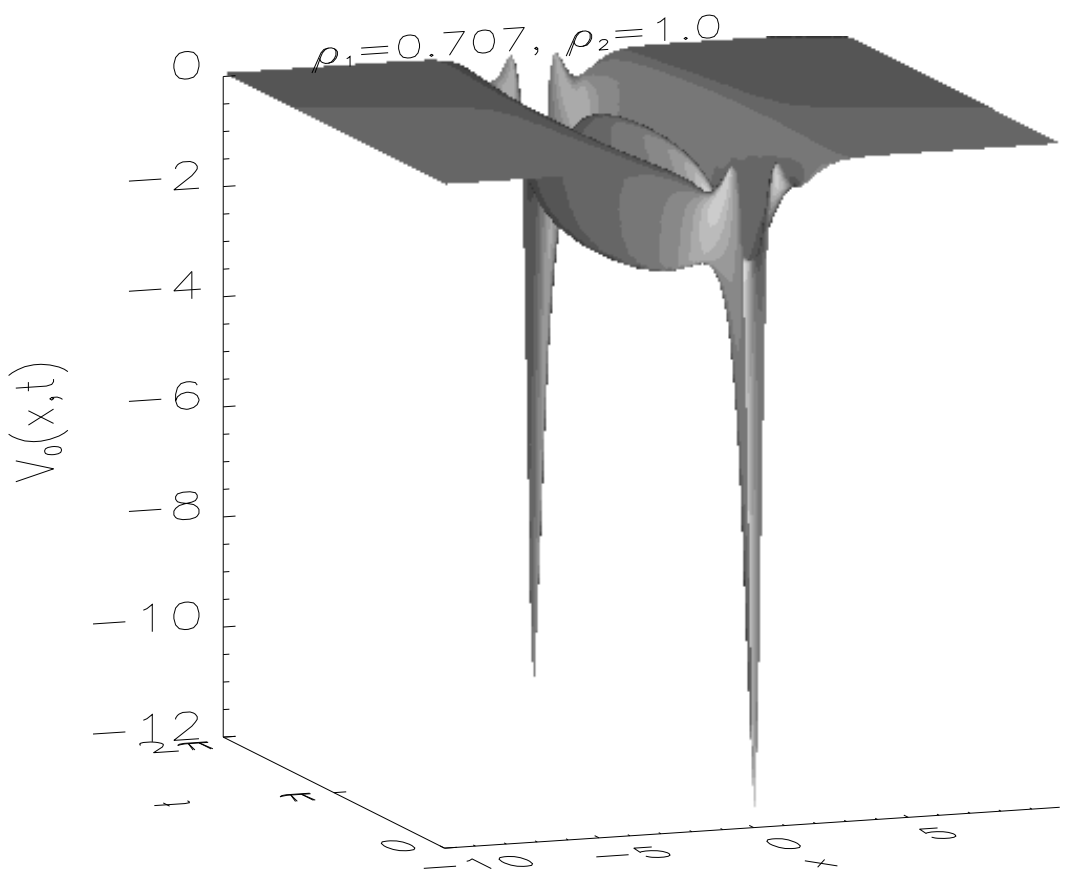

Figure 1. The potential well $V_{0}(x, t)$ for $\rho_{1}=1 / \sqrt{2}$ and $\rho_{2}=1$. The phase parameters are $\theta_{1}=\theta_{2}=0$. This well is time-periodic with period $L=2 \pi$ and even in $x$.

in which the first term is even in $x$ and the second term is odd in $x$. Also, it is clear that $a(-x, t, \lambda)=a(x, t,-\lambda)$.

A particularly convenient orthonormal basis of the two-dimensional space of bound states is given by the formulae

$$
\begin{aligned}
& \Psi_{\mathrm{b}}^{(\mathrm{e})}(x, t)=\frac{1}{\sqrt{4\left(\rho_{1}+\rho_{2}\right)}}\left[\frac{2}{\rho_{1}-\rho_{2}} a\left(x, t,-\mathrm{i} \rho_{1}\right)+\frac{2}{\rho_{2}-\rho_{1}} a\left(x, t,-\mathrm{i} \rho_{2}\right)\right] \\
& \Psi_{\mathrm{b}}^{(\mathrm{o})}(x, t)=\frac{1}{\sqrt{4\left(\rho_{1}+\rho_{2}\right)}}\left[\sqrt{\frac{\rho_{2}}{\rho_{1}}} \frac{2}{\rho_{1}-\rho_{2}} a\left(x, t,-\mathrm{i} \rho_{1}\right)+\sqrt{\frac{\rho_{1}}{\rho_{2}}} \frac{2}{\rho_{2}-\rho_{1}} a\left(x, t,-\mathrm{i} \rho_{2}\right)\right] .
\end{aligned}
$$

In this case, the even symmetry of the potential $V_{0}(x, t)$ guarantees that we may choose one basis element to be even and the other to be odd; we are using superscripts '(e)' and '(o)' to refer to even and odd functions of $x$, respectively. These bound state solutions of the linear Schrödinger equation are shown in figures 3 and 4 . The two bound state modes are Bloch functions in $t$, with the same Floquet multiplier $\exp \left(2 \mathrm{i} \beta_{\mathrm{b}} L\right)=\exp \left(2 \mathrm{i} \rho_{1}^{2} L\right)=\exp \left(2 \mathrm{i} \rho_{2}^{2} L\right)$. Note that, in reference to the remark made at the end of section 2.1, the function $\Psi_{\mathrm{b}}^{(\mathrm{e})}(x, t)$ is proportional to $\psi=2 \mathrm{i} b^{(1)}(x, t)$, which is a two-soliton solution of the nonlinear Schrödinger equation

$$
\mathrm{i} \partial_{t} \psi+\frac{1}{2} \partial_{x}^{2} \psi+|\psi|^{2} \psi=0
$$

Correspondingly, $V_{0}(x, t)=-|\psi|^{2}$ is the self-consistent potential. 


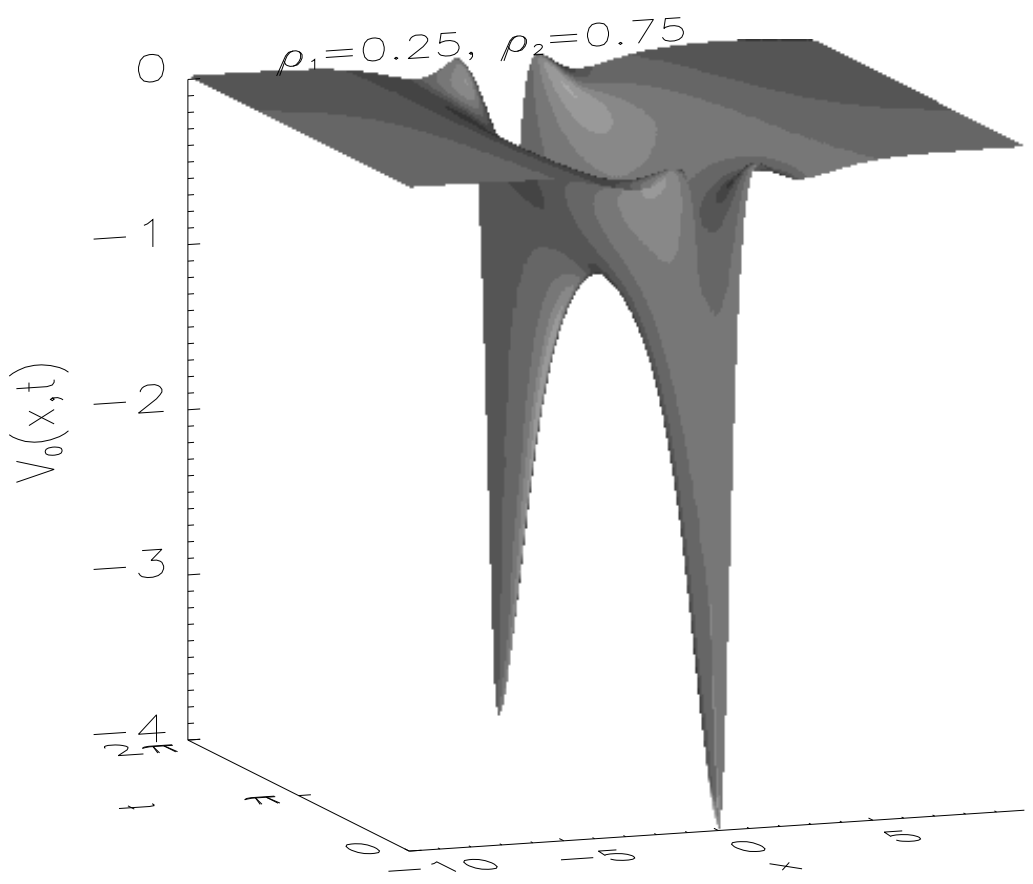

Figure 2. The potential well $V_{0}(x, t)$ for $\rho_{1}=\frac{1}{4}$ and $\rho_{2}=\frac{3}{4}$. The phase parameters are $\theta_{1}=\theta_{2}=0$. This well is time-periodic with period $L=2 \pi$ and even in $x$.

It will also be useful to decompose the continuum into odd and even parts. Using the fact that $a(x, t,-\lambda)=a(-x, t, \lambda)$, define

$$
\begin{aligned}
& \Psi_{\mathrm{d}}^{(\mathrm{e})}(x, t, \lambda) \doteq \frac{1}{\sqrt{2 \pi\left(\lambda^{2}+\rho_{1}^{2}\right)\left(\lambda^{2}+\rho_{2}^{2}\right)}}(a(x, t, \lambda)+a(x, t,-\lambda)) \\
& \Psi_{\mathrm{d}}^{(\mathrm{o})}(x, t, \lambda) \doteq \frac{1}{\sqrt{2 \pi\left(\lambda^{2}+\rho_{1}^{2}\right)\left(\lambda^{2}+\rho_{2}^{2}\right)}}(a(x, t, \lambda)-a(x, t,-\lambda))
\end{aligned}
$$

where $\lambda \geqslant 0$. The solutions $\Psi_{\mathrm{d}}^{\mathrm{e}}(x, t, \lambda)$ and $\Psi_{\mathrm{d}}^{(\mathrm{o})}(x, t, \lambda)$ are also Bloch functions in $t$ of period $L$ with Floquet multiplier $\exp \left(-2 \mathrm{i} \lambda^{2} L\right)$.

These solutions of the unperturbed problem have the following inner products [22]:

$$
\begin{aligned}
& \left\langle\Psi_{\mathrm{b}}^{(\mathrm{e})}(\cdot, t), \Psi_{\mathrm{d}}^{(\mathrm{e})}(\cdot, t, \lambda)\right\rangle=0 \\
& \left\langle\Psi_{\mathrm{b}}^{(\mathrm{e})}(\cdot, t), \Psi_{\mathrm{b}}^{(\mathrm{e})}(\cdot, t)\right\rangle=1 \\
& \left\langle\Psi_{\mathrm{d}}^{(\mathrm{e})}(\cdot, t, \lambda), \Psi_{\mathrm{d}}^{(\mathrm{e})}(\cdot, t, \eta)\right\rangle=\delta(\lambda-\eta) .
\end{aligned}
$$

In this latter relation it is assumed that both $\lambda$ and $\eta$ are positive real. Similar relations hold among the odd solutions, and of course everything even is orthogonal to everything odd. If the perturbation $W(x, t)$ is also even in $x$, then this observation will allow us to treat the even and odd parts of the field $f(x, t)$ in isolation to all orders in the perturbation theory.

In our subsequent analysis of the coupled-mode equations for this family of periodic potentials, we shall assume that the perturbation $W(x, t)$ is also an even function of $x$, and thus restrict attention to the subspace of initial conditions, $f(x, 0)$ which are either even or 
(a)

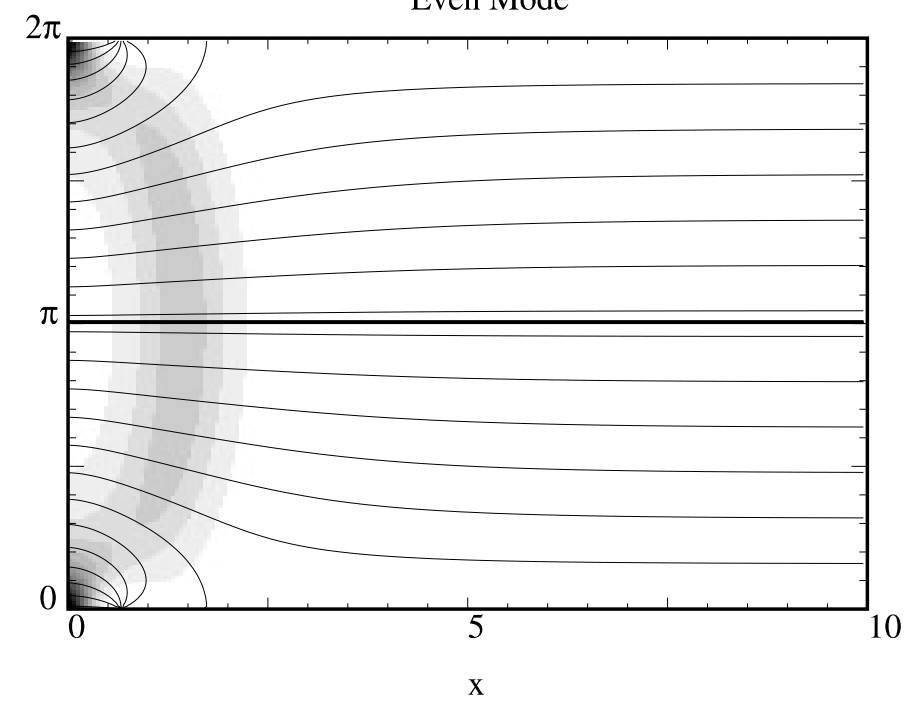

Even Mode

(b)

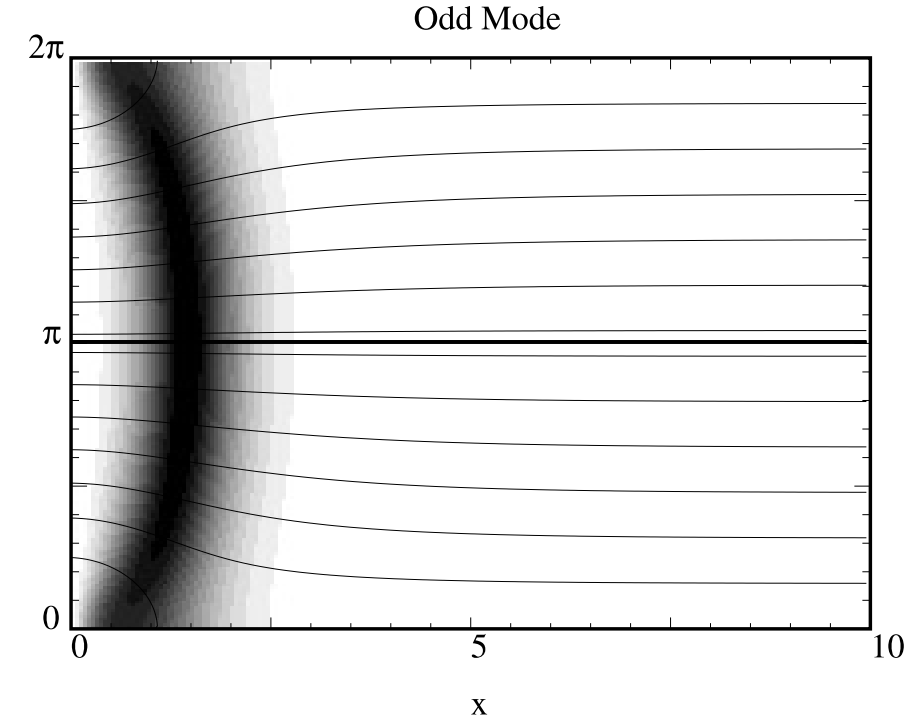

Figure 3. Equal phase contours for the even $(a)$ and odd $(b)$ modes superimposed on a density plot of the corresponding square modulus. The parameter values are $\rho_{1}=1 / \sqrt{2}$ and $\rho_{2}=1$. For these values of $\rho_{1}$ and $\rho_{2}$, the Floquet multiplier is equal to 1 , and therefore these are periodic functions of $t$.

odd in $x$. By the spatial symmetry of $V=V_{0}+W, f(x, t)$ has the same parity as $f(x, 0)$. In analogy with the above derivation of coupled-mode equations, we can then expand $f(x, t)$ in terms of (even or odd) modes of the unperturbed problem

$$
f(x, t)=B_{\mathrm{b}}^{(\alpha)}(t) \Psi_{\mathrm{b}}^{(\alpha)}(x, t)+\int_{0}^{\infty} B_{\mathrm{d}}^{(\alpha)}(t, \lambda) \Psi_{\mathrm{d}}^{(\alpha)}(x, t, \lambda) \mathrm{d} \lambda
$$

where $\alpha=\mathrm{e}$ if $f(x, 0)$ is even and $\alpha=\mathrm{o}$ if $f(x, 0)$ is odd. Coupled-mode equations for the amplitudes $B_{\mathrm{b}}^{(\alpha)}(t), B_{\mathrm{d}}^{(\alpha)}(t, \lambda), \lambda \in \mathbb{R}$ analogous to those derived in the absence of 
(a)
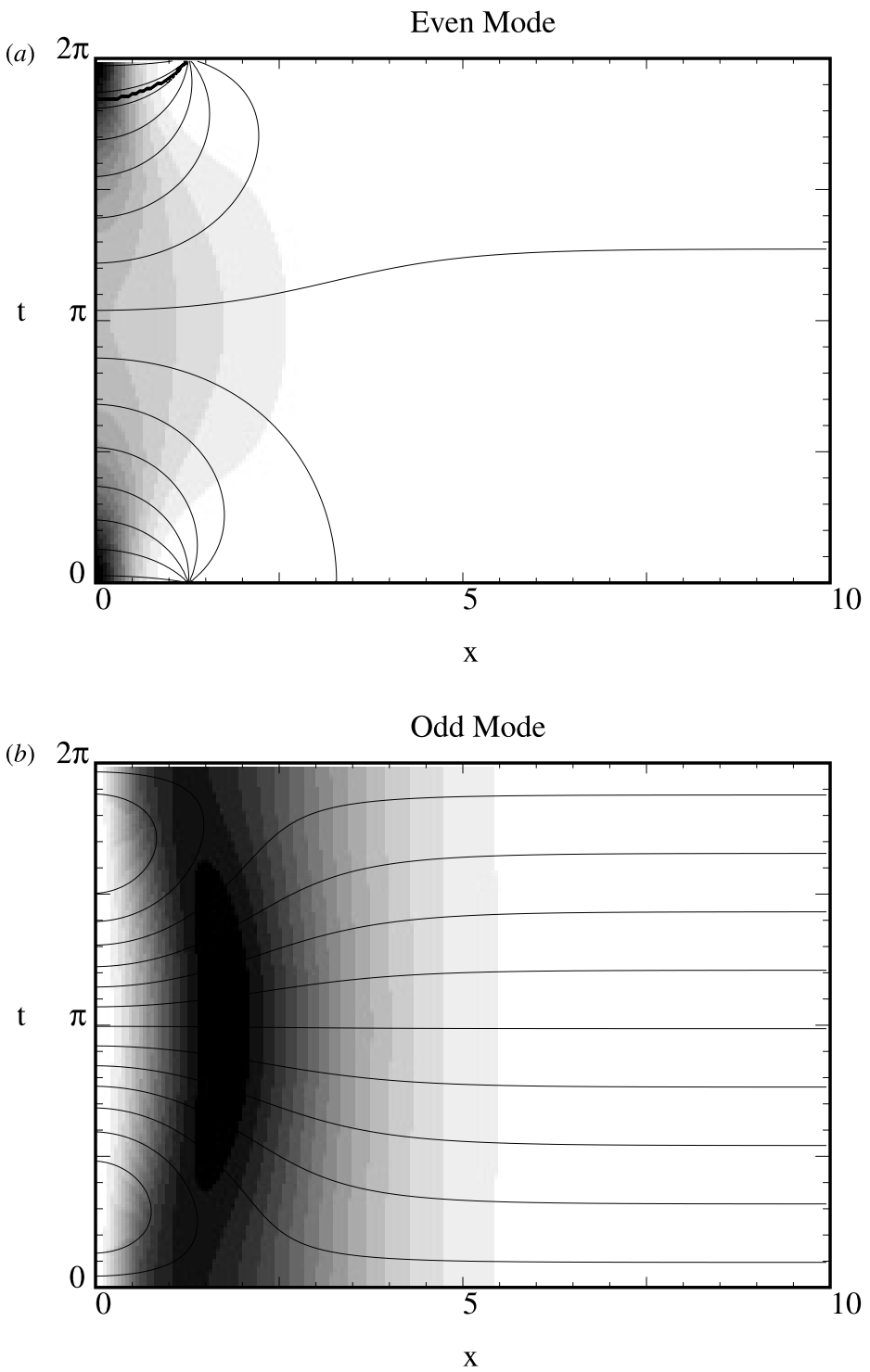

Figure 4. Equal phase contours for the even $(a)$ and odd $(b)$ modes superimposed on a density plot of the corresponding square modulus. The parameter values are $\rho_{1}=\frac{1}{4}$ and $\rho_{2}=\frac{3}{4}$. Here, the Floquet multiplier is not equal to 1 , and the modes are not periodic in $t$, although they have Bloch form.

any particular symmetry can then be derived by projecting the dynamical system (2.14) onto these even and odd basis modes. In section 3 we show, by using the Floquet factorization of the unitary evolution associated with the unperturbed dynamics, that these coupled-mode equations can be re-expressed as the following system (cf the system (3.15)) which is more 
amenable to our techniques:

$\mathrm{i} \partial_{t} A_{\mathrm{b}}(t)+2 \beta_{\mathrm{b}} A_{\mathrm{b}}(t)=M(t) A_{\mathrm{b}}(t)+\int_{0}^{\infty} N^{(\mathrm{p})}(t, \lambda) A_{\mathrm{d}}(t, \lambda) \mathrm{d} \lambda$

$\mathrm{i} \partial_{t} A_{\mathrm{d}}(t, \eta)-2 \eta^{2} A_{\mathrm{d}}(t, \eta)=N^{(\mathrm{p})}(t, \eta)^{*} A_{\mathrm{b}}(t)+\int_{0}^{\infty} K^{(\mathrm{p})}(t, \eta, \lambda) A_{\mathrm{d}}(t, \lambda) \mathrm{d} \lambda$

where

$$
A_{\mathrm{b}}(t) \doteq B_{\mathrm{b}}(t) \mathrm{e}^{2 \mathrm{i} \beta_{\mathrm{b}} t} \quad A_{\mathrm{d}}(t, \lambda) \doteq B_{\mathrm{d}}(t, \lambda) \mathrm{e}^{-2 \mathrm{i} \lambda^{2} t}
$$

and where the scalar coefficients, all periodic with period $L$, are

$$
\begin{aligned}
& M(t)=\left\langle\Psi_{\mathrm{b}}(\cdot, t), W(\cdot, t) \Psi_{\mathrm{b}}(\cdot, t)\right\rangle \\
& N^{(\mathrm{p})}(t, \lambda)=\left\langle\Psi_{\mathrm{b}}(\cdot, t), W(\cdot, t) \Psi_{\mathrm{d}}(\cdot, t, \lambda)\right\rangle \mathrm{e}^{2 \mathrm{i}\left(\lambda^{2}+\beta_{\mathrm{b}}\right) t} \\
& K^{(\mathrm{p})}(t, \eta, \lambda)=\left\langle\Psi_{\mathrm{d}}(\cdot, t, \eta), W(\cdot, t) \Psi_{\mathrm{d}}(\cdot, t, \lambda)\right\rangle \mathrm{e}^{2 \mathrm{i}\left(\lambda^{2}-\eta^{2}\right) t} .
\end{aligned}
$$

Remark. To avoid cumbersome formulae, we have omitted the superscripts (o) and (e), with the understanding that the amplitudes correspond to either one type or the other, depending on the parity of $f(x, 0)$.

The system (2.31) may be viewed as that governing a family of oscillators: a single discrete oscillator whose amplitude is $A_{\mathrm{b}}(t)$ coupled to a continuum of oscillators with amplitudes $A_{\mathrm{d}}(t, \eta), \eta \in \mathbb{R}_{+}$.

In section 4 we shall analyse the coupled-mode system (2.31), and determine the detailed asymptotic behaviour of its solutions for small $W(x, t)$ over different time scales.

\section{Coupled-mode equations for periodic potentials}

Consider a dynamical system of the form (1.6), where both the unperturbed and the perturbed potential are time periodic with the same period $L$. We will encounter a concrete example of such a problem in section 5. Floquet theory [1] suggests the introduction of a new timeperiodic basis, with respect to which the problem (2.14) becomes a periodic perturbation of an autonomous Hamiltonian system. This change of basis transforms the problem at hand into one similar to that treated in $[15,28]$. Similar methods are used along with resonance theory in a weakly nonlinear setting in [25].

\subsection{Floquet factorization}

Let $\mathcal{U}(t)$ denote the unitary evolution operator (or propagator) of the unperturbed problem, so that for any $L^{2}(\mathbb{R})$ function $f(x), f(x, t)=\mathcal{U}(t) f(x)$ is the solution of the unperturbed problem with $f(x, 0)=f(x)$. As a consequence of the periodicity, the evolution operator can be factored into two operators on $L^{2}(\mathbb{R})$

$$
\mathcal{U}(t)=\mathcal{P}(t) \mathrm{e}^{-\mathrm{i} t \mathcal{B}}
$$

where $\mathcal{P}(t+L)=\mathcal{P}(t)$ and $\mathcal{B}$ is independent of $t$. This factorization can be motivated by the observation that by periodicity, there is an operator $\mathcal{M}$ satisfying $\mathcal{U}(t+L)=\mathcal{U}(t) \mathcal{M}$, and that by setting $t=0$, in fact, one has $\mathcal{M}=\mathcal{U}(L)$. Since $\mathcal{U}(L)$ is unitary, one can find a self-adjoint operator $\mathcal{B}$ such that $\mathcal{M}=\mathcal{U}(L)=\mathrm{e}^{-\mathrm{i} L \mathcal{B}}$. This operator $\mathcal{B}$, in turn, defines the Abelian unitary group $\mathrm{e}^{-\mathrm{i} t \mathcal{B}}$. Now it is easy to see that $\mathcal{P}(t)=\mathcal{U}(t) \mathrm{e}^{\mathrm{i} t \mathcal{B}}$ is a unitary operator satisfying $\mathcal{P}(t+L)=\mathcal{P}(t)$. 
Let $\mathcal{W}(t)$ be the operator of multiplication by the correction to the potential $W(x, t)$, and set $y(x, t)=\mathcal{P}(t)^{\dagger} f(x, t)$. Then, the perturbed equation (1.6) becomes

$$
\mathrm{i} \partial_{t} y-\mathcal{B} y=\tilde{\mathcal{W}}(t) y
$$

where $\tilde{\mathcal{W}}(t) \doteq \mathcal{P}(t)^{\dagger} \mathcal{W}(t) \mathcal{P}(t)$, a 'dressing' of $\mathcal{W}(t)$. The form of (3.2) is similar to the type of problem treated in [15, 28]. The 'unperturbed Hamiltonian' $\mathcal{B}$ is time-independent, and selfadjoint. The perturbation $\tilde{\mathcal{W}}(t)$ is localized, self-adjoint and time-periodic because the periods of $\mathcal{P}(t)$ and $\mathcal{W}(t)$ are equal. Typically the perturbation contains frequency components at all overtones of the fundamental frequency, and thus the version of the theory described in [15] is most appropriate. The key qualitative difference between the present situation and that treated in [15] is that here the unperturbed operator can have multiple bound states. We will soon introduce a symmetry that removes this difficulty from the scope of this paper. However, the methods of $[15,28]$ can be extended to give results on radiation damping due to the coupling of multiple discrete modes to the continuum for a general class of spatially localized and time-dependent perturbations [16].

In fact, one can simplify the problem even further by invoking the spectral theorem for the self-adjoint operator $\mathcal{B}$. This guarantees the existence of an isomorphism $\mathcal{V}: L^{2}(\mathbb{R}) \rightarrow$ $L^{2}(\Sigma, \mathrm{d} \mu)$ to the space of square-integrable functions on some set $\Sigma$ with measure $\mathrm{d} \mu$, such that $\mathcal{V B}=\mathcal{T} \mathcal{V}$ where $\mathcal{T}$ is a real diagonal operator on $L^{2}(\Sigma, \mathrm{d} \mu)$ (i.e. an operator of multiplication by a bounded function from $\Sigma$ to $\mathbb{R})$. Setting $z(t)=\mathcal{V} y(t)$, we find the equation

$$
\mathrm{i} \partial_{t} z-\mathcal{T} z=\mathcal{V} \tilde{\mathcal{W}}(t) \mathcal{V}^{\dagger} z
$$

In quantum mechanics, making the transformation from (2.14) to (3.3) to facilitate the study of perturbations is known as going from the Schrödinger picture to the interaction picture.

In the particular example we will analyse in detail, arising from even perturbations of the two-soliton even potential described at the end of section 2, the operator $\mathcal{B}$ has a single degenerate eigenvalue of $-2 \beta_{\mathrm{b}}<0$ of geometric multiplicity two. By restricting separately to even and odd spaces of initial conditions (which is possible because the potential $V_{0}(x, t)+W(x, t)$ is symmetric in $\left.x\right)$, the problem is reduced to one which, formally, is precisely of the type studied in [15]. We may then apply the methods developed in [15] (subject to some appropriate hypotheses) without modification.

We now use our explicit knowledge developed in section 2 of the unitary propagator $\mathcal{U}(t)$ corresponding to a time-periodic separable potential $V_{0}(x, t)$ to find the operators $\mathcal{P}(t)$ and $\mathcal{B}$, and then to diagonalize $\mathcal{B}$. This effectively implements the programme described above and casts the perturbed problem (2.14) into a form (3.3) more suitable for analysis. We begin with the observation that each element of the basis of solutions of the unperturbed problem is a Bloch function or Floquet mode. We have

$$
\Psi_{\mathrm{d}}(x, t+L, \lambda)=\mathrm{e}^{-2 \mathrm{i} \lambda^{2} L} \Psi_{\mathrm{d}}(x, t, \lambda)
$$

where $\lambda$ is arbitrary real. Also, we have

$$
a\left(x, t+L,-\mathrm{i} \rho_{k}\right)=\mathrm{e}^{2 \mathrm{i} \rho_{k}^{2} L} a\left(x, t,-\mathrm{i} \rho_{k}\right) .
$$

Note that the commensurability relations (2.11) imply quite generally that the Floquet multipliers $\exp \left(2 \mathrm{i} \rho_{k}^{2} L\right)$ are all equal. This generalizes the observation made above in the context of the two-soliton potentials. This means that the entire $M$-dimensional subspace of bound states consists of degenerate Floquet modes. In particular, the elements of any 
orthonormal basis $\left\{\Psi_{\mathrm{b}, k}(x, t), k=1, \ldots, M\right\}$ have the same Floquet multiplier $\dagger \exp \left(2 \mathrm{i} \beta_{\mathrm{b}} L\right)$. It now follows from (3.4) and (3.5) that the functions defined by

$$
\begin{aligned}
& \Psi_{\mathrm{d}}^{(\mathrm{p})}(x, t, \lambda) \doteq \mathrm{e}^{2 \mathrm{i} \lambda^{2} t} \Psi_{\mathrm{d}}(x, t, \lambda) \\
& \Psi_{\mathrm{b}, k}^{(\mathrm{p})}(x, t) \doteq \mathrm{e}^{-2 \mathrm{i} \beta_{\mathrm{b}} t} \Psi_{\mathrm{b}, k}(x, t)
\end{aligned}
$$

are time-periodic with period $L$, as denoted by the superscript '(p)'.

As described in section 2, the solution of the unperturbed problem with initial data $f(x)$ is expanded as

$$
\begin{aligned}
f(x, t)= & \mathcal{U}(t) f(x) \\
= & \sum_{k=1}^{M}\left\langle\Psi_{\mathrm{b}, k}(\cdot, 0), f(\cdot)\right\rangle \Psi_{\mathrm{b}, k}(x, t)+\int_{-\infty}^{\infty}\left\langle\Psi_{\mathrm{d}}(\cdot, 0, \lambda), f(\cdot)\right\rangle \Psi_{\mathrm{d}}(x, t, \lambda) \mathrm{d} \lambda \\
= & \sum_{k=1}^{M}\left\langle\Psi_{\mathrm{b}, k}^{(\mathrm{p})}(\cdot, 0), \mathrm{e}^{2 \mathrm{i} \beta_{\mathrm{b}} t} f(\cdot)\right\rangle \Psi_{\mathrm{b}, k}^{(\mathrm{p})}(x, t) \\
& +\int_{-\infty}^{\infty}\left\langle\Psi_{\mathrm{d}}^{(\mathrm{p})}(\cdot, 0, \lambda), \mathrm{e}^{-2 \mathrm{i}^{2} t} f(\cdot)\right\rangle \Psi_{\mathrm{d}}^{(\mathrm{p})}(x, t, \lambda) \mathrm{d} \lambda .
\end{aligned}
$$

We now use the completeness relation at $t=0$ to factor $\mathcal{U}(t)$ as $\mathcal{P}(t) \mathrm{e}^{-\mathrm{i} t \mathcal{B}}$ where

$$
\begin{aligned}
\mathrm{e}^{-\mathrm{i} t \mathcal{B}} f(x)= & \sum_{k=1}^{M}\left\langle\Psi_{\mathrm{b}, k}^{(\mathrm{p})}(\cdot, 0), \mathrm{e}^{2 \mathrm{i} \beta_{\mathrm{b}} t} f(\cdot)\right\rangle \Psi_{\mathrm{b}, k}^{(\mathrm{p})}(x, 0) \\
& +\int_{-\infty}^{\infty}\left\langle\Psi_{\mathrm{d}}^{(\mathrm{p})}(\cdot, 0, \lambda), \mathrm{e}^{-2 \mathrm{i} \lambda^{2} t} f(\cdot)\right\rangle \Psi_{\mathrm{d}}^{(\mathrm{p})}(x, 0, \lambda) \mathrm{d} \lambda
\end{aligned}
$$

and

$$
\mathcal{P}(t) g(x)=\sum_{k=1}^{M}\left\langle\Psi_{\mathrm{b}, k}^{(\mathrm{p})}(\cdot, 0), g(\cdot)\right\rangle \Psi_{\mathrm{b}, k}^{(\mathrm{p})}(x, t)+\int_{-\infty}^{\infty}\left\langle\Psi_{\mathrm{d}}^{(\mathrm{p})}(\cdot, 0, \lambda), g(\cdot)\right\rangle \Psi_{\mathrm{d}}^{(\mathrm{p})}(x, t, \lambda) \mathrm{d} \lambda .
$$

We have used, several times, the fact that at $t=0$ there is no distinction between the basis elements and their periodic counterparts defined by (3.6). It is easy to see that $f(x, t)=\mathcal{U}(t) f(x)=\mathcal{P}(t) \mathrm{e}^{-\mathrm{i} t \mathcal{B}} f(x)$ is the solution of the unperturbed initial-value problem with data $f(x) \in L^{2}(\mathbb{R})$ and that $\mathcal{P}(t)$ is periodic with period $L$ and $\mathcal{U}(L)=\mathrm{e}^{-\mathrm{i} L \mathcal{B}}$.

The generator of the Abelian unitary group $\mathrm{e}^{-\mathrm{i} t \mathcal{B}}$ is

$$
\begin{aligned}
\mathcal{B} f(x) & =\left.\mathrm{i} \frac{\mathrm{d}}{\mathrm{d} t} \mathrm{e}^{-\mathrm{i} t \mathcal{B}} f(x)\right|_{t=0} \\
& =\sum_{k=1}^{M}\left\langle\Psi_{\mathrm{b}, k}^{(\mathrm{p})}(\cdot, 0),-2 \beta_{\mathrm{b}} f(\cdot)\right\rangle \Psi_{\mathrm{b}, k}^{(\mathrm{p})}(x, 0)+\int_{-\infty}^{\infty}\left\langle\Psi_{\mathrm{d}}^{(\mathrm{p})}(\cdot, 0, \lambda), 2 \lambda^{2} f(\cdot)\right\rangle \Psi_{\mathrm{d}}^{(\mathrm{p})}(x, 0, \lambda) \mathrm{d} \lambda \\
& =\sum_{k=1}^{M}\left\langle\Psi_{\mathrm{b}, k}(\cdot, 0),-2 \beta_{\mathrm{b}} f(\cdot)\right\rangle \Psi_{\mathrm{b}, k}(x, 0)+\int_{-\infty}^{\infty}\left\langle\Psi_{\mathrm{d}}(\cdot, 0, \lambda), 2 \lambda^{2} f(\cdot)\right\rangle \Psi_{\mathrm{d}}(x, 0, \lambda) \mathrm{d} \lambda .
\end{aligned}
$$

In the last step we have dropped the superscripts '(p)' since everything is evaluated at $t=0$. This formula for the self-adjoint operator $\mathcal{B}$ makes clear its spectral decomposition. The

$\dagger$ Recall that the Floquet exponents are not unique but that the Floquet multipliers are. Identification of the Floquet exponents with a single number $\beta_{\mathrm{b}}>0$ amounts to a particular choice of branch of the logarithm. 
isomorphism $\mathcal{V}$ takes a function $g(x) \in L^{2}(\mathbb{R})$ to a function $A_{\mathrm{d}}(\lambda)$ for $\lambda \in \mathbb{R}$ and a set of $M$ numbers $A_{\mathrm{b}, k}$ for $k=1, \ldots, M$ defined by

$$
A_{\mathrm{d}}(\lambda) \doteq\left\langle\Psi_{\mathrm{d}}^{(\mathrm{p})}(\cdot, 0, \lambda), g(\cdot)\right\rangle
$$

and for $k=1, \ldots, M$,

$$
A_{\mathrm{b}, k} \doteq\left\langle\Psi_{\mathrm{b}, k}^{(\mathrm{p})}(\cdot, 0), g(\cdot)\right\rangle .
$$

The diagonal operator $\mathcal{T}$ is then simply defined by

$$
\mathcal{T}\left[\begin{array}{c}
A_{\mathrm{d}}(\lambda) \\
A_{\mathrm{b}, 1} \\
\vdots \\
A_{\mathrm{b}, M}
\end{array}\right]=\left[\begin{array}{cccc}
2 \lambda^{2} & & & \\
& -2 \beta_{\mathrm{b}} & & \\
& & \ddots & \\
& & & -2 \beta_{\mathrm{b}}
\end{array}\right]\left[\begin{array}{c}
A_{\mathrm{d}}(\lambda) \\
A_{\mathrm{b}, 1} \\
\vdots \\
A_{\mathrm{b}, M}
\end{array}\right] .
$$

It is now easy to use the definition of the unitary periodic operator $\mathcal{P}(t)$ and the unitary isomorphism $\mathcal{V}$, along with the completeness relation to compute the dressed operator $\mathcal{V} \tilde{\mathcal{W}}(t) \mathcal{V}^{\dagger}$ and thus write the perturbed problem (2.14) in the simple form (3.3). The dynamical unknowns are in the range of $\mathcal{V}$, the space $L^{2}(\Sigma, \mathrm{d} \mu)$, and are given in terms of $f(x, t)$, the solution of (2.14), by

$$
\begin{aligned}
& A_{\mathrm{b}, k}=\left(\mathcal{V P}(t)^{\dagger} f(\cdot, t)\right)_{\mathrm{b}, k}=\left\langle\Psi_{\mathrm{b}, k}^{(\mathrm{p})}(\cdot, t), f(\cdot, t)\right\rangle \\
& A_{\mathrm{d}}(\lambda)=\left(\mathcal{V P}(t)^{\dagger} f(\cdot, t)\right)_{\mathrm{d}}(\lambda)=\left\langle\Psi_{\mathrm{d}}^{(\mathrm{p})}(\cdot, t, \lambda), f(\cdot, t)\right\rangle .
\end{aligned}
$$

When $f(x, t)$ satisfies (2.14), these quantities satisfy the system

$$
\begin{aligned}
& \mathrm{i} \partial_{t} \vec{A}_{\mathrm{b}}+2 \beta_{\mathrm{b}} \vec{A}_{\mathrm{b}}=M(t) \vec{A}_{\mathrm{b}}+\int_{-\infty}^{\infty} A_{\mathrm{d}}(\lambda) \vec{N}^{(\mathrm{p})}(t, \lambda) \mathrm{d} \lambda \\
& \mathrm{i} \partial_{t} A_{\mathrm{d}}(\eta)-2 \eta^{2} A_{\mathrm{d}}(\eta)=\vec{N}^{(\mathrm{p})}(t, \eta)^{\dagger} \vec{A}_{\mathrm{b}}+\int_{-\infty}^{\infty} K^{(\mathrm{p})}(t, \eta, \lambda) A_{\mathrm{d}}(\lambda) \mathrm{d} \lambda
\end{aligned}
$$

where $\vec{A}_{\mathrm{b}}$ is the vector of components $A_{\mathrm{b}, 1}, \ldots, A_{\mathrm{b}, M}$, and the time-periodic matrix elements are defined in terms of (2.17) by

$$
\begin{aligned}
& \vec{N}^{(\mathrm{p})}(t, \lambda) \doteq \mathrm{e}^{2 \mathrm{i}\left(\lambda^{2}+\beta_{\mathrm{b}}\right) t} \vec{N}(t, \lambda) \\
& K^{(\mathrm{p})}(t, \eta, \lambda) \doteq \mathrm{e}^{2 \mathrm{i}\left(\lambda^{2}-\eta^{2}\right) t} K(t, \eta, \lambda) .
\end{aligned}
$$

The periodicity of these matrix elements when $W(x, t)$ is periodic with period $L$ is also clear from these explicit formulae and the Bloch relations (3.4) and (3.5) for the basis of solutions; these imply similar ones for the matrix elements defined by (2.17). We have

$$
\begin{aligned}
& M(t+L)=M(t) \\
& \vec{N}(t+L, \lambda)=\mathrm{e}^{-2 \mathrm{i}\left(\lambda^{2}+\beta_{\mathrm{b}}\right) L} \vec{N}(t, \lambda) \\
& K(t+L, \eta, \lambda)=\mathrm{e}^{2 \mathrm{i}\left(\eta^{2}-\lambda^{2}\right) L} K(t, \eta, \lambda) .
\end{aligned}
$$

Of course, the right-hand side of (3.15) is just the operator $\mathcal{V} \tilde{\mathcal{W}}(t) \mathcal{V}^{\dagger}$ operating on the dynamical unknowns. Similarly, the perturbation operator $\tilde{\mathcal{W}}(t)$ operating in the space $L^{2}(\mathbb{R})$ can be 
written explicitly as

$$
\begin{aligned}
& \tilde{\mathcal{W}}(t) f(x)=\sum_{k=1}^{M} \sum_{l=1}^{M} M_{k, l}(t)\left\langle\Psi_{\mathrm{b}, l}(\cdot, 0), f(\cdot)\right\rangle \Psi_{\mathrm{b}, k}(x, 0) \\
&+\sum_{k=1}^{M} \int_{-\infty}^{\infty} N_{k}^{(\mathrm{p})}(t, \eta)\left\langle\Psi_{\mathrm{d}}(\cdot, 0, \eta), f(\cdot)\right\rangle \Psi_{\mathrm{b}, k}(x, 0) \mathrm{d} \eta \\
&+\sum_{l=1}^{M} \int_{-\infty}^{\infty} N_{l}^{(\mathrm{p})}(t, \lambda)^{*}\left\langle\Psi_{\mathrm{b}, l}(\cdot, 0), f(\cdot)\right\rangle \Psi_{\mathrm{d}}(x, 0, \lambda) \mathrm{d} \lambda \\
&+\int_{-\infty}^{\infty} \int_{-\infty}^{\infty} K^{(\mathrm{p})}(t, \lambda, \eta)\left\langle\Psi_{\mathrm{d}}(\cdot, 0, \eta), f(\cdot)\right\rangle \Psi_{\mathrm{d}}(x, 0, \lambda) \mathrm{d} \lambda \mathrm{d} \eta .
\end{aligned}
$$

For the special choice of $V_{0}(x, t)$ discussed at the end of section 2, evenness implies that there is one bound state of each parity. If the perturbation $W(x, t)$ also has even symmetry in $x$, the coupled-mode system (3.15) reduces to a system of the type (2.31) when the initial condition is restricted to either even or odd parity. It is easily checked that the unknowns as defined above correspond exactly to those defined in section 2 for the system (2.31).

\section{Analysis of the coupled-mode equations}

In this section we study the structural instability of the even and odd breather modes introduced at the end of section 2 associated with the two-soliton time-periodic even potentials. We first give a simple argument valid for short times that in the presence of a perturbation $W(x, t)$ to the potential $V_{0}(x, t)$, the bound state begins to decay initially. We then seek to capture the dynamics for longer times, primarily to show that this initial phase of decay does not reverse itself, but takes on a different, exponentially decaying, character. The decay will be first calculated formally, using asymptotic expansions and the method of multiple scales. Then, using the results of Kirr and Weinstein [15], we show that, at least in the odd case, it is possible to make statements about the decay process that are valid globally in time. In particular, these arguments will rigorously justify the formal results for the odd case, and will show that the exponential decay model is only a valid approximation until it becomes smaller than the dispersive part of the solution. The bound state ultimately dies algebraically in time, qualitatively indistinguishable from the dispersive components of the solution to which it is orthogonal.

\subsection{Small-time analysis. The watched pot effect}

A simple calculation carried out at the level of the coupled-mode equations (2.31) shows that the effect of the perturbation is to cause the bound state to decay immediately both forward and backward in time. More complicated calculations will be required to show that the decay does not stop or reverse for longer times, although it takes on a different character. The approach in the small-time analysis is simply to expand the solution in Taylor series

$$
\begin{aligned}
& A_{\mathrm{b}}(t)=A_{\mathrm{b}}(0)+c_{1} t+c_{2} t^{2}+\mathrm{O}\left(t^{3}\right) \\
& A_{\mathrm{d}}(\lambda, t)=d_{1}(\lambda) t+\mathrm{O}\left(t^{2}\right)
\end{aligned}
$$


and use the (known) Taylor expansions of the matrix elements, in particular,

$$
\begin{aligned}
& M(t)=M(0)+M^{\prime}(0) t+\mathrm{O}\left(t^{2}\right) \\
& N^{(\mathrm{p})}(t, \lambda)=N^{(\mathrm{p})}(0, \lambda)+\mathrm{O}(t) .
\end{aligned}
$$

Substituting these series into (2.31), one finds

$$
\begin{gathered}
{\left[\mathrm{i} c_{1}+2 \beta_{\mathrm{b}} A(0)-M(0) A_{\mathrm{b}}(0)\right]+\left[2 \mathrm{i} c_{2}+2 \beta_{\mathrm{b}} c_{1}-M(0) c_{1}-M^{\prime}(0) A_{\mathrm{b}}(0)\right.} \\
\left.-\int_{0}^{\infty} d_{1}(\lambda) N^{(\mathrm{p})}(0, \lambda) \mathrm{d} \lambda\right] t=\mathrm{O}\left(t^{2}\right) \\
\mathrm{i} d_{1}(\eta)-N^{(\mathrm{p})}(0, \eta)^{*} A_{\mathrm{b}}(0)=\mathrm{O}(t) .
\end{gathered}
$$

Solving for $c_{1}$ and $c_{2}$ yields an approximation for $A_{\mathrm{b}}(t)$, valid for small $t$

$$
\begin{aligned}
A_{\mathrm{b}}(t)=A_{\mathrm{b}}(0) & {\left[1-\mathrm{i}\left(M(0)-2 \beta_{\mathrm{b}}\right) t\right.} \\
& \left.-\frac{1}{2}\left(\mathrm{i} M^{\prime}(0)+\left(M(0)-2 \beta_{\mathrm{b}}\right)^{2}+\int_{0}^{\infty}\left|N^{(\mathrm{p})}(0, \lambda)\right|^{2} \mathrm{~d} \lambda\right) t^{2}+\mathrm{O}\left(t^{3}\right)\right] .
\end{aligned}
$$

It easily follows that

$$
\left|A_{\mathrm{b}}(t)\right|^{2}=\left|A_{\mathrm{b}}(0)\right|^{2}\left[1-t^{2} \int_{0}^{\infty}\left|N^{(\mathrm{p})}(0, \lambda)\right|^{2} \mathrm{~d} \lambda+\mathrm{O}\left(t^{3}\right)\right] .
$$

Note that the smallness of the perturbation is not exploited in these calculations. This Taylor expansion shows that the initial phase of the evolution is a process of radiative decay, since $\left|A_{\mathrm{b}}(t)\right|^{2}<\left|A_{\mathrm{b}}(0)\right|^{2}$ for all non-zero $t$ in some neighbourhood of $t=0$. The decay is symmetric in time.

The fact that the decay is an order $\mathrm{O}\left(t^{2}\right)$ effect is quite general $\dagger$ and is well known in the perturbation theory of stationary Schrödinger equations. It has an interesting interpretation in the quantum theory of ideal measurements, the so-called 'watched pot effect'. Suppose that an ideal measurement is made at some point during the evolution of the wavefunction to determine whether the state is bound, and the measurement yields a positive result. The probability of

$\dagger$ In the general setting, the decay is a simple consequence of the Cauchy-Schwarz inequality. One supposes that $\mathcal{U}(t)$ is the unitary propagator of the possibly time-dependent unperturbed problem:

$$
\mathrm{i} \mathcal{U}_{t}(t) \phi^{0}=\mathcal{H}_{0}(t) \mathcal{U}(t) \phi^{0}
$$

for all states $\phi^{0}$. One then considers the perturbed equation

$$
\mathrm{i} \psi_{t}=\left(\mathcal{H}_{0}(t)+\mathcal{W}(t)\right) \psi
$$

by setting $\psi(t)=\mathcal{U}(t) \phi(t)$, giving the 'interaction picture' equation

$$
\mathrm{i} \phi_{t}=\mathcal{U}(t)^{\dagger} \mathcal{W}(t) \mathcal{U}(t) \phi
$$

which one solves by Taylor series in $t$. The result is

$$
\phi(t)=\left(\mathcal{I}-\mathrm{i} \mathcal{W}(0) t+\frac{1}{2} t^{2}\left(-\mathrm{i} \mathcal{W}^{\prime}(0)-\mathcal{W}(0)^{2}+\left[\mathcal{H}_{0}(0), \mathcal{W}(0)\right]\right)+\mathrm{O}\left(t^{3}\right)\right) \phi(0)
$$

The probability of remaining in the unperturbed state is then found to be (using self-adjointness of both $\mathcal{W}(0)$ and $\left.\mathcal{H}_{0}(0)\right)$

$$
\begin{aligned}
|\langle\mathcal{U}(t) \phi(0), \mathcal{U}(t) \phi(t)\rangle|^{2} & =|\langle\phi(0), \phi(t)\rangle|^{2} \\
& =\|\phi(0)\|_{2}^{4}-\left(\|\mathcal{W}(0) \phi(0)\|_{2}^{2}\|\phi(0)\|_{2}^{2}-|\langle\phi(0), \mathcal{W}(0) \phi(0)\rangle|^{2}\right) t^{2}+\mathrm{O}\left(t^{3}\right) .
\end{aligned}
$$

This quantity is initially decreasing in time as a consequence of the Cauchy-Schwarz inequality. 
a positive result at time $t$ is $\left|A_{\mathrm{b}}(t)\right|^{2} /\left|A_{\mathrm{b}}(0)\right|^{2}$. The theory of ideal measurements says that as a consequence of the measurement disturbing the system, the wavefunction 'collapses' upon a positive result to the bound state, and evolution of the wavefunction according to the Schrödinger equation continues from this 'reset' bound state. One may then try to determine the asymptotic effect of making many such measurements in a finite time interval. In particular, we can ask about the limiting probability of finding the system in the bound state after each of $n$ ideal measurements performed at times $t_{n}=T / n$, as $n \rightarrow \infty$. After each positive result, the wavefunction collapses and the experiment is restarted. The Schrödinger evolution takes place over short time intervals so it is appropriate to replace the probability in each interval $p(t)$ by its short-time approximation $p(t)=1-(\alpha t)^{2}+\mathrm{O}\left(t^{3}\right)$. The $n$ measurements are independent events, so the probability of always finding the system bound after each measurement is simply

$$
P_{n}=p(T / n)^{n} .
$$

Because the 'time slice' decay probability $1-p(t)$ is quadratic in $t, P_{n}$ tends to unity $\dagger$ as $n \rightarrow \infty$, regardless of the value of $T$. So if the measurements are performed infinitely often, the decay of the bound state never occurs. The quantum 'watched pot' never boils.

\subsection{Multiple-scales analysis}

We begin the multiple-scales analysis by assuming that the correction $W(x, t)=W(x, t ; \epsilon)$ to the potential energy has an expansion in a small parameter, $\epsilon$ (see, for example, equation (5.11)),

$$
W(x, t ; \epsilon)=\epsilon W_{1}(x, t)+\mathrm{O}\left(\epsilon^{2}\right) .
$$

It then follows that the coupling coefficient functions in (2.31) have formal expansions for small $\epsilon$,

$$
\begin{aligned}
& M(t)=\epsilon M_{1}(t)+\epsilon^{2} M_{2}(t)+\mathrm{O}\left(\epsilon^{3}\right) \\
& N^{(\mathrm{p})}(t, \lambda)=\epsilon N_{1}^{(\mathrm{p})}(t, \lambda)+\mathrm{O}\left(\epsilon^{2}\right) \\
& K^{(\mathrm{p})}(t, \eta, \lambda)=\epsilon K_{1}^{(\mathrm{p})}(t, \eta, \lambda)+\mathrm{O}\left(\epsilon^{2}\right) .
\end{aligned}
$$

Here, $M_{1}(t), N_{1}^{(\mathrm{p})}(t)$ and $K_{1}^{(\mathrm{p})}(t, \eta, \lambda)$ correspond to the expressions for $M(t), N^{(\mathrm{p})}$ and $K^{(\mathrm{p})}$ in (2.33) with $W$ replaced by $W_{1}$.

The amplitudes $A_{\mathrm{b}}(t ; \epsilon)$ and $A_{\mathrm{d}}(t, \lambda ; \epsilon)$ are assumed to have asymptotic expansions of the form

$A_{\mathrm{b}}(t ; \epsilon)=A_{\mathrm{b}}^{(0)}\left(T_{0}, T_{1}, T_{2}, \ldots\right)+\epsilon A_{\mathrm{b}}^{(1)}\left(T_{0}, T_{1}, T_{2}, \ldots\right)+\epsilon^{2} A_{\mathrm{b}}^{(2)}\left(T_{0}, T_{1}, T_{2}, \ldots\right)+\mathrm{O}\left(\epsilon^{3}\right)$

$A_{\mathrm{d}}(t, \lambda ; \epsilon)=A_{\mathrm{d}}^{(0)}\left(T_{0}, T_{1}, T_{2}, \ldots, \lambda\right)+\epsilon A_{\mathrm{d}}^{(1)}\left(T_{0}, T_{1}, T_{2}, \ldots, \lambda\right)+\mathrm{O}\left(\epsilon^{2}\right)$

where the $T_{k} \doteq \epsilon^{k} t$ are time scale variables. Such expansions of given functions $A_{\mathrm{b}}(t ; \epsilon)$ and $A_{\mathrm{d}}(t, \lambda ; \epsilon)$ are highly non-unique. However, the guiding principle of the method of multiple scales (see, for example, [12]) stipulates that the dependence of the various terms on the 'slow' times $T_{1}, T_{2}$, and so on is chosen so that each term is uniformly bounded as a function of the 'fast' time $T_{0}$. This procedure is quite systematic, and is supposed to keep the error terms in any truncation uniformly small in time intervals where $T_{k}$ is bounded for some $k$ as $\epsilon$ tends to

$\dagger$ The superlinear nature of the decay probability is important. If $p(t)=1-|\alpha t|+\mathrm{O}\left(t^{2}\right)$, then $P_{n}$ tends to $\mathrm{e}^{-|\alpha T|}<1$ instead. 
zero. We will see by comparison with the rigorous results that this formal procedure indeed works as advertised.

One now substitutes these expansions into the system (2.31) and expands the time derivative operating on the expansion coefficients in (4.9) according to the chain rule,

$$
\partial_{t}=\partial_{T_{0}}+\epsilon \partial_{T_{1}}+\epsilon^{2} \partial_{T_{2}}+\cdots
$$

The coupling coefficients, all being periodic functions of $t$ with period $L$ independent of $\epsilon$, are taken to be explicit functions of $t=T_{0}$ only. Substituting them into (2.31) along with the expansions (4.9) and the chain rule formula (4.10), and equating terms with the same powers of $\epsilon$ leads to a hierarchy of equations:

$$
\begin{aligned}
& \mathrm{O}(1):\left\{\begin{array}{l}
\mathrm{i} \partial_{T_{0}} A_{\mathrm{b}}^{(0)}+2 \beta_{\mathrm{b}} A_{\mathrm{b}}^{(0)}=0 \\
\mathrm{i} \partial_{T_{0}} A_{\mathrm{d}}^{(0)}(\eta)-2 \eta^{2} A_{\mathrm{d}}^{(0)}(\eta)=0
\end{array}\right. \\
& \mathrm{O}(\epsilon):\left\{\begin{array}{l}
\mathrm{i} \partial_{T_{0}} A_{\mathrm{b}}^{(1)}+2 \beta_{\mathrm{b}} A_{\mathrm{b}}^{(1)}=-\mathrm{i} \partial_{T_{1}} A_{\mathrm{b}}^{(0)}+M_{1}\left(T_{0}\right) A_{\mathrm{b}}^{(0)}+\int_{0}^{\infty} N_{1}^{(\mathrm{p})}\left(T_{0}, \lambda\right) A_{\mathrm{d}}^{(0)}(\lambda) \mathrm{d} \lambda \\
\mathrm{i} \partial_{T_{0}} A_{\mathrm{d}}^{(1)}(\eta)-2 \eta^{2} A_{\mathrm{d}}^{(1)}(\eta)=-\mathrm{i} \partial_{T_{1}} A_{\mathrm{d}}^{(0)}(\eta)+N_{1}^{(\mathrm{p})}\left(T_{0}, \eta\right)^{*} A_{\mathrm{b}}^{(0)} \\
\quad+\int_{0}^{\infty} K_{1}^{(\mathrm{p})}\left(T_{0}, \eta, \lambda\right) A_{\mathrm{d}}^{(0)}(\lambda) \mathrm{d} \lambda
\end{array}\right. \\
& \mathrm{O}\left(\epsilon^{2}\right):\left\{\begin{array}{l}
\mathrm{i} \partial_{T_{0}} A_{\mathrm{b}}^{(2)}+2 \beta_{\mathrm{b}} A_{\mathrm{b}}^{(2)}=-\mathrm{i} \partial_{T_{1}} A_{\mathrm{b}}^{(1)}-\mathrm{i} \partial_{T_{2}} A_{\mathrm{b}}^{(0)}+M_{2}\left(T_{0}\right) A_{\mathrm{b}}^{(0)}+M_{1}\left(T_{0}\right) A_{\mathrm{b}}^{(1)} \\
\quad+\int_{0}^{\infty} N_{1}^{(\mathrm{p})}\left(T_{0}, \lambda\right) A_{\mathrm{d}}^{(1)}(\lambda) \mathrm{d} \lambda+\int_{0}^{\infty} N_{2}^{(\mathrm{p})}\left(T_{0}, \lambda\right) A_{\mathrm{d}}^{(0)}(\lambda) \mathrm{d} \lambda \\
\mathrm{i} \partial_{T_{0}} A_{\mathrm{d}}^{(2)}(\eta)-2 \eta^{2} A_{\mathrm{d}}^{(2)}(\eta)=\cdots
\end{array}\right.
\end{aligned}
$$

and so on. Our initial conditions are encoded in the expansions (4.9) as $A_{\mathrm{b}}^{(0)}(0,0,0, \ldots)=A_{\mathrm{b} 0}$, $A_{\mathrm{d}}^{(0)}(0,0,0, \ldots, \lambda)=0$, and for $j \geqslant 1, A_{\mathrm{b}}^{(j)}(0,0,0, \ldots)=A_{\mathrm{d}}^{(j)}(0,0,0, \ldots, \lambda)=0$. We now proceed to solve the hierarchy sequentially.

Solving equations (4.11) at order $\mathrm{O}(1)$ subject to the initial conditions gives

$$
A_{\mathrm{b}}^{(0)}=C \mathrm{e}^{2 \mathrm{i} \beta_{\mathrm{b}} T_{0}} \quad A_{\mathrm{d}}^{(0)}(\eta)=0
$$

where $C=C\left(T_{1}, T_{2}, \ldots\right)$ satisfies the initial condition $C(0,0, \ldots)=A_{\mathrm{b} 0}$ but is otherwise undetermined at this stage.

In the first of the two equations (4.12) appearing at $\mathrm{O}(\epsilon)$, it is natural to make the substitution

$$
A_{\mathrm{b}}^{(1)}=f \mathrm{e}^{2 \mathrm{i} \beta_{\mathrm{b}} T_{0}}
$$

which leads to the equation

$$
\partial_{T_{0}} f=-\partial_{T_{1}} C-\mathrm{i} M_{1}\left(T_{0}\right) C .
$$

Integrating with the use of the initial condition $f\left(T_{0}=0\right)=0$, and keeping in mind that $T_{1}$ and $T_{0}$ are to be thought of as independent variables, leads to the expression for $A_{\mathrm{b}}^{(1)}$,

$$
A_{\mathrm{b}}^{(1)}=\left(-\partial_{T_{1}} C \cdot T_{0}-\mathrm{i} C \int_{0}^{T_{0}} M_{1}(s) \mathrm{d} s\right) \mathrm{e}^{2 \mathrm{i} \beta_{\mathrm{b}} T_{0}}
$$


We need this correction to be bounded as a function of $T_{0}$ so that the asymptotic expansion will be well-ordered for long times. Since $M_{1}(s)$ is a periodic function of period $L$, this requirement uniquely determines $\partial_{T_{1}} C$,

$$
\partial_{T_{1}} C=-\mathrm{i} \overline{M_{1}} C \quad \overline{M_{1}} \doteq \frac{1}{L} \int_{0}^{L} M_{1}(s) \mathrm{d} s .
$$

Thus,

$$
C=D \mathrm{e}^{-\mathrm{i} \overline{M_{1}} T_{1}} \quad D=D\left(T_{2}, \ldots\right) \quad D(0, \ldots)=A_{\mathrm{b} 0} .
$$

Putting together what we have for the bound state amplitude at this time,

$$
A_{\mathrm{b}}^{(0)}=D \mathrm{e}^{-\mathrm{i} \overline{M_{1}} T_{1}} \mathrm{e}^{2 \mathrm{i} \beta_{\mathrm{b}} T_{0}} \quad A_{\mathrm{b}}^{(1)}=-\mathrm{i} D \mathrm{e}^{-\mathrm{i} \overline{M_{1}} T_{1}} \mathrm{e}^{2 \mathrm{i} \beta_{\mathrm{b}} T_{0}} \int_{0}^{T_{0}}\left(M_{1}(s)-\overline{M_{1}}\right) \mathrm{d} s .
$$

This has been the first application in our calculation of the guiding principle of the method of multiple scales, that dependence of expansion terms on 'slow' times is chosen to ensure that the expansion terms are uniformly bounded with respect to the 'fast' time $T_{0}$. Now we solve for the correction to the dispersive mode amplitude at this order (in fact, the leading term) using the second of equations (4.12). Substituting the expressions from the previous order and using the initial conditions gives a unique expression

$$
A_{\mathrm{d}}^{(1)}(\eta)=-\mathrm{i} D \mathrm{e}^{-\mathrm{i} \overline{M_{1}} T_{1}} \int_{0}^{T_{0}} N_{1}^{(\mathrm{p})}(s, \eta)^{*} \mathrm{e}^{2 \mathrm{i} \beta_{\mathrm{b}} s} \mathrm{e}^{-2 \mathrm{i} \eta^{2}\left(T_{0}-s\right)} \mathrm{d} s .
$$

Continuing systematically with equation (4.13) for the bound state amplitude correction at order $\mathrm{O}\left(\epsilon^{2}\right)$, we substitute all the expressions known thus far and observe the utility of the change of variables

$$
A_{\mathrm{b}}^{(2)}=h \mathrm{e}^{-\mathrm{i} \overline{M_{1}} T_{1}} \mathrm{e}^{2 \mathrm{i} \beta_{\mathrm{b}} T_{0}} .
$$

We find for $h$ the simple equation

$\partial_{T_{0}} h=-D \cdot\left(M_{1}\left(T_{0}\right)-\overline{M_{1}}\right) \int_{0}^{T_{0}}\left(M_{1}(s)-\overline{M_{1}}\right) \mathrm{d} s-\partial_{T_{2}} D-D \cdot \gamma\left(T_{0}\right)$

where

$$
\gamma\left(T_{0}\right) \doteq \mathrm{i} M_{2}\left(T_{0}\right)+\int_{0}^{\infty} N_{1}^{(\mathrm{p})}\left(T_{0}, \lambda\right) \int_{0}^{T_{0}} \mathrm{e}^{-2 \mathrm{i}\left(\lambda^{2}+\beta_{\mathrm{b}}\right)\left(T_{0}-s\right)} N_{1}^{(\mathrm{p})}(s, \lambda)^{*} \mathrm{~d} s \mathrm{~d} \lambda .
$$

Equation (4.23) can be analysed as follows. By linearity, we can express $h$ as a sum: $h=h_{1}+h_{2}$, where

$$
\begin{aligned}
& \partial_{T_{0}} h_{1}=-D \cdot\left(M_{1}\left(T_{0}\right)-\overline{M_{1}}\right) \int_{0}^{T_{0}}\left(M_{1}(s)-\overline{M_{1}}\right) \mathrm{d} s \\
& \partial_{T_{0}} h_{2}=-\partial_{T_{2}} D-D \cdot \gamma\left(T_{0}\right)
\end{aligned}
$$

and where we assume the initial conditions $h_{1}\left(T_{0}=0\right)=h_{2}\left(T_{0}=0\right)=0$. Integrating the equation for $h_{1}$ exactly using the initial condition gives

$$
h_{1}=-\frac{1}{2} D\left(\int_{0}^{T_{0}}\left(M_{1}(s)-\overline{M_{1}}\right) \mathrm{d} s\right)^{2}
$$

which is periodic, and in particular bounded, by periodicity of $M_{1}\left(T_{0}\right)$. 
We now want to select the dependence of $D$ on the slow time $T_{2}$ such that $h_{2}$, as found from the second of equations (4.25), is a bounded function of $T_{0}$. Using the initial condition to integrate the equation for $h_{2}$ with respect to $T_{0}$ while holding $T_{2}$ fixed gives

$$
h_{2}=-T_{0} \partial_{T_{2}} D-D \int_{0}^{T_{0}} \gamma(s) \mathrm{d} s
$$

Clearly, the possibility of choosing $D\left(T_{2}\right)$ so that expression (4.27) is bounded in $T_{0}$ depends on the behaviour of $\gamma\left(T_{0}\right)$ in the limits $T_{0} \rightarrow \pm \infty$. We now study $\gamma\left(T_{0}\right)$ for large $\left|T_{0}\right|$.

We can compute the $s$-integral in (4.24) exactly if we introduce the Fourier series for the periodic function $N_{1}^{(\mathrm{p})}\left(T_{0}, \lambda\right)$,

$$
N_{1}^{(\mathrm{p})}\left(T_{0}, \lambda\right)=\sum_{k=-\infty}^{\infty} N_{1, k}(\lambda) \mathrm{e}^{2 \pi \mathrm{i} k T_{0} / L}
$$

Note that in terms of the Fourier coefficients of $N^{(\mathrm{p})}\left(T_{0}, \lambda ; \epsilon\right)$ itself (see (4.8)), we have

$$
N_{1, k}(\lambda)=\lim _{\epsilon \rightarrow 0} \epsilon^{-1} N_{k}(\lambda ; \epsilon) .
$$

Substituting the Fourier series into (4.24), integrating term by term with respect to $s$, and changing variables to $\sigma=\lambda^{2}$, we arrive at

$$
\gamma\left(T_{0}\right)=\mathrm{i} M_{2}\left(T_{0}\right)+\sum_{n, k=-\infty}^{\infty} \mathrm{i} \int_{0}^{\infty} \frac{N_{1, n}(\sqrt{\sigma}) N_{1, k}(\sqrt{\sigma})^{*}}{4 \sqrt{\sigma}\left(\sigma-\sigma_{k}\right)}\left[\mathrm{e}^{-2 \mathrm{i}\left(\sigma-\sigma_{n}\right) T_{0}}-\mathrm{e}^{2 \pi \mathrm{i}(n-k) T_{0} / L}\right] \mathrm{d} \sigma
$$

where the resonances $\sigma_{n}$ are defined by

$$
\sigma_{n} \doteq \pi n / L-\beta_{\mathrm{b}}
$$

Note that for all terms having $\sigma_{k}>0$, the difference of the exponentials in the integrand vanishes for $\sigma=\sigma_{k}$, so there is no singularity. Moreover, the Fourier coefficients $N_{1, n}(\lambda)$ are by construction analytic functions of $\lambda$ for $\lambda$ in a sector including the real axis, and so the quantities $N_{1, n}(\sqrt{\sigma})$ are analytic in a neighbourhood of the positive real $\sigma$-axis. This property extends to the whole integrand, and we may therefore deform the integration contour away from the real axis in an effort to study the behaviour for large $\left|T_{0}\right|$ by a steepest-descents-type argument.

For positive $T_{0}$, we deform the contour into the lower half-plane. For $\delta>0$, let $C_{+}^{\delta}$ be the contour consisting of the diagonal segment from 0 to $(1-\mathrm{i}) \delta$ followed by the horizontal ray from $(1-\mathrm{i}) \delta$ to $-\mathrm{i} \delta+\infty$ (see figure 5$)$. We have

$$
\begin{aligned}
\gamma\left(T_{0}\right)= & \mathrm{i} M_{2}\left(T_{0}\right)+\sum_{n, k=-\infty}^{\infty} \mathrm{i} \int_{C_{+}^{\delta}} \frac{N_{1, n}(\sqrt{\sigma}) N_{1, k}(\sqrt{\sigma})^{*}}{4 \sqrt{\sigma}\left(\sigma-\sigma_{k}\right)}\left[\mathrm{e}^{-2 \mathrm{i}\left(\sigma-\sigma_{n}\right) T_{0}}-\mathrm{e}^{2 \pi \mathrm{i}(n-k) T_{0} / L}\right] \mathrm{d} \sigma \\
= & \mathrm{i} M_{2}\left(T_{0}\right)+\sum_{n, k=-\infty}^{\infty} \mathrm{i} \int_{C_{+}^{\delta}} \frac{N_{1, n}(\sqrt{\sigma}) N_{1, k}(\sqrt{\sigma})^{*}}{4 \sqrt{\sigma}\left(\sigma-\sigma_{k}\right)} \mathrm{e}^{-2 \mathrm{i}\left(\sigma-\sigma_{n}\right) T_{0}} \mathrm{~d} \sigma \\
& -\sum_{n, k=-\infty}^{\infty} \mathrm{i} \int_{C_{+}^{\delta}} \frac{N_{1, n}(\sqrt{\sigma}) N_{1, k}(\sqrt{\sigma})^{*}}{4 \sqrt{\sigma}\left(\sigma-\sigma_{k}\right)} \mathrm{e}^{2 \pi \mathrm{i}(n-k) T_{0} / L} \mathrm{~d} \sigma \\
= & \gamma_{0}\left(T_{0}\right)+\gamma_{1}^{+}\left(T_{0}\right)+\gamma_{2}^{+}\left(T_{0}\right)
\end{aligned}
$$



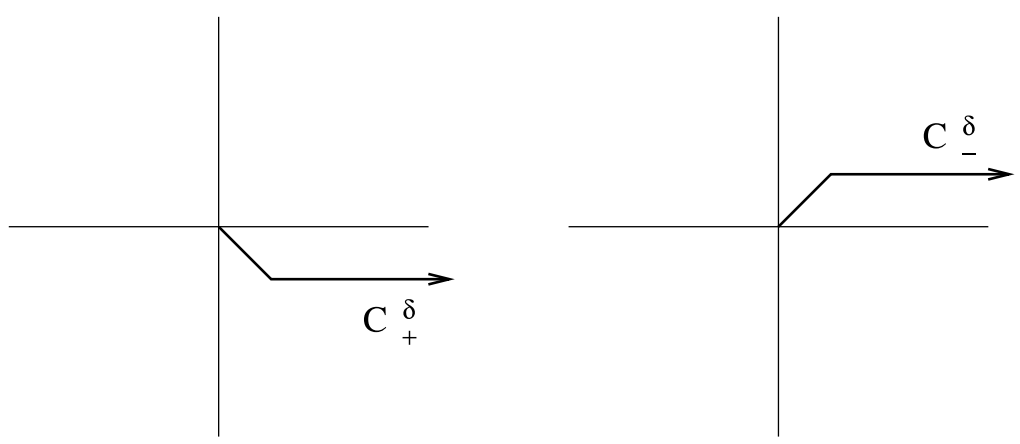

Figure 5. The deformed integration contours $C_{+}^{\delta}$ and $C_{-}^{\delta}$.

so that on the new contour $C_{+}^{\delta}$ the two integrals converge independently. The term $\gamma_{0}\left(T_{0}\right)$ is periodic in $T_{0}$ with period $L$ and mean value $\mathrm{i} \overline{M_{2}}$. The term $\gamma_{2}^{+}\left(T_{0}\right)$ is also a periodic function of $T_{0}$ with period $L$. Its mean value is given by the terms in the sum with $n=k$,

$$
\overline{\gamma_{2}^{+}}=-\sum_{n=-\infty}^{\infty} \mathrm{i} \int_{C_{+}^{\delta}} \frac{\left|N_{1, n}(\sqrt{\sigma})\right|^{2}}{4 \sqrt{\sigma}\left(\sigma-\sigma_{n}\right)} \mathrm{d} \sigma .
$$

Letting $\delta$ tend to zero does not alter the value of the integral, and then we may use the PlemeljSokhotski formula $\dagger$ to evaluate the terms with $\sigma_{n}>0$ to find

$$
\overline{\gamma_{0}+\gamma_{2}^{+}}=\mathrm{i} \overline{M_{2}}-\mathrm{i} \Lambda_{2}+\Gamma_{2}
$$

where

$$
\Lambda_{2} \doteq \sum_{n=-\infty}^{n_{0}-1} \int_{0}^{\infty} \frac{\left|N_{1, n}(\sqrt{\sigma})\right|^{2} \mathrm{~d} \sigma}{4 \sqrt{\sigma}\left(\sigma-\sigma_{n}\right)}+\sum_{n=n_{0}}^{\infty} \mathrm{PV} \int_{0}^{\infty} \frac{\left|N_{1, n}(\sqrt{\sigma})\right|^{2} \mathrm{~d} \sigma}{4 \sqrt{\sigma}\left(\sigma-\sigma_{n}\right)}
$$

and

$$
\Gamma_{2} \doteq \frac{\pi}{4} \sum_{n=n_{0}}^{\infty} \frac{\left|N_{1, n}\left(\sqrt{\sigma_{n}}\right)\right|^{2}}{\sqrt{\sigma_{n}}} .
$$

Finally, consider the term $\gamma_{1}^{+}\left(T_{0}\right)$. Its time integral, calculated term by term, is

$$
\begin{aligned}
\int_{0}^{T_{0}} \gamma_{1}^{+}(s) \mathrm{d} s= & -\sum_{n, k=-\infty}^{\infty} \int_{C_{+}^{\delta}} \frac{N_{1, n}(\sqrt{\sigma}) N_{1, k}(\sqrt{\sigma})^{*}}{8 \sqrt{\sigma}\left(\sigma-\sigma_{k}\right)\left(\sigma-\sigma_{n}\right)}\left[\mathrm{e}^{-2 \mathrm{i}\left(\sigma-\sigma_{n}\right) T_{0}}-1\right] \mathrm{d} \sigma \\
= & \sum_{n, k=-\infty}^{\infty} \int_{C_{+}^{\delta}} \frac{N_{1, n}(\sqrt{\sigma}) N_{1, k}(\sqrt{\sigma})^{*}}{8 \sqrt{\sigma}\left(\sigma-\sigma_{k}\right)\left(\sigma-\sigma_{n}\right)} \mathrm{d} \sigma \\
& -\sum_{n, k=-\infty}^{\infty} \int_{C_{+}^{\delta}} \frac{N_{1, n}(\sqrt{\sigma}) N_{1, k}(\sqrt{\sigma})^{*}}{8 \sqrt{\sigma}\left(\sigma-\sigma_{k}\right)\left(\sigma-\sigma_{n}\right)} \mathrm{e}^{-2 \mathrm{i}\left(\sigma-\sigma_{n}\right) T_{0}} \mathrm{~d} \sigma .
\end{aligned}
$$

The first term is independent of $T_{0}$ (and also of $\delta>0$, since the integral converges and the integrand is analytic). In the second term, the real part of the exponent is negative for $T_{0}>0$, so

$\dagger$ This is merely the distributional identity

$$
(x \pm \mathrm{i} 0)^{-1}=\mathrm{PV} x^{-1} \mp \mathrm{i} \pi \delta(x) .
$$


for $T_{0}$ large and positive, the integrand is exponentially small except in a small neighbourhood of $\sigma=0$. This small neighbourhood gives a leading contribution to the integrand that is $\mathrm{O}\left(T_{0}^{-1 / 2}\right)$, and in particular is bounded for large $T_{0}>0$.

Putting these results together, we find that for large $T_{0}>0$, we have

$$
\int_{0}^{T_{0}} \gamma(s) \mathrm{d} s=\left(\mathrm{i} \overline{M_{2}}-\mathrm{i} \Lambda_{2}+\Gamma_{2}\right) T_{0}+\mathrm{O}(1) .
$$

Going back to (4.27), it is clear that choosing

$$
\partial_{T_{2}} D=-\left(\mathrm{i} \overline{M_{2}}-\mathrm{i} \Lambda_{2}+\Gamma_{2}\right) D
$$

will lead to a solution $h_{2}\left(T_{0}\right)$ that is uniformly bounded for all $T_{0}>0$. Also note that the first term in $\gamma\left(T_{0}\right)$ contributes a term

$$
h_{2, M} \doteq-\mathrm{i} \int_{0}^{T_{0}}\left(M_{2}(s)-\overline{M_{2}}\right) \mathrm{d} s
$$

to the expression for $h_{2}\left(T_{0}\right)$. We write $h_{2}\left(T_{0}\right)=h_{2, M}\left(T_{0}\right)+\tilde{h}_{2}\left(T_{0}\right)$.

To find the behaviour of $\gamma\left(T_{0}\right)$ and its time integral as $T_{0}$ tends to $-\infty$, we repeat the above steps, this time deforming the integration contour into the upper half-plane to facilitate the steepest-descents argument. The path of integration is now $C_{-}^{\delta}$ (see figure 5). The only difference is in the sign of $\Gamma_{2}$; the correct choice for a bounded solution for all $T_{0}$ is

$$
\partial_{T_{2}} D=-\left(\mathrm{i} \overline{M_{2}}-\mathrm{i} \Lambda_{2}+\operatorname{sgn}\left(T_{0}\right) \Gamma_{2}\right) D .
$$

Thus, the method of multiple scales gives the following approximation to the bound state mode amplitude:

$$
\begin{aligned}
A_{\mathrm{b}}(t)=A_{\mathrm{b} 0} \mathrm{e}^{2 \mathrm{i} \beta_{\mathrm{b}} t} \mathrm{e}^{-\mathrm{i}\left(\epsilon \overline{M_{1}}+\epsilon^{2} \overline{M_{2}}\right) t} \mathrm{e}^{\mathrm{i} \epsilon^{2} \Lambda_{2} t} \mathrm{e}^{-\epsilon^{2} \Gamma_{2}|t|}\left(1-\mathrm{i} \epsilon \int_{0}^{t}\left(M_{1}(s)-\overline{M_{1}}\right) \mathrm{d} s\right. \\
\left.\quad-\frac{1}{2} \epsilon^{2}\left(\int_{0}^{t}\left(M_{1}(s)-\overline{M_{1}}\right) \mathrm{d} s\right)^{2}-\mathrm{i} \epsilon^{2} \int_{0}^{t}\left(M_{2}(s)-\overline{M_{2}}\right) \mathrm{d} s+\epsilon^{2} \tilde{h}_{2}(t)+\mathrm{O}\left(\epsilon^{3}\right)\right) .
\end{aligned}
$$

It is not hard to see that an asymptotically equivalent expression is just

$$
A_{\mathrm{b}}(t)=A_{\mathrm{b} 0} \mathrm{e}^{2 \mathrm{i} \beta_{\mathrm{b}} t} \mathrm{e}^{-\epsilon^{2} \Gamma_{2}|t|} \mathrm{e}^{\mathrm{i} \epsilon^{2} \Lambda_{2} t} \mathrm{e}^{-\mathrm{i} \int_{0}^{t} M(s) \mathrm{d} s}\left(1+\mathrm{O}\left(\epsilon^{2}\right)\right)
$$

This asymptotic formula is expected to be uniformly valid as $\epsilon$ tends to zero for all $|t|<K \epsilon^{-2}$ for any constant $K$.

So the behaviour of the bound state amplitude under the influence of a periodic perturbation, as predicted by the multiple-scale theory, is dominated by two effects, a shift in frequency accompanied by exponential decay. The shift in frequency is an order $O(\epsilon)$ effect, coming from $\bar{M}$. This shift can be traced back to the influence of the perturbation directly on the bound state; there is no coupling to any other modes in this term. The order $\mathrm{O}\left(\epsilon^{2}\right)$ effects include both a further adjustment to the frequency through the quantity $\epsilon^{2} \Lambda_{2}$, the Lamb shift, and exponential decay through the quantity $\epsilon^{2} \Gamma_{2}$. Clearly, these two numbers are the real and imaginary parts of the same complex frequency. Unlike the leading-order phase shift, both of these effects are clearly due to the resonant coupling between the bound state and the continuum that is introduced and mediated by the periodic perturbation. Due to the exponential decay, the lifetime of the bound state is seen to be approximately $\epsilon^{-2} / \Gamma_{2}$, which is quite long for small $\epsilon$. For this reason, under small perturbations of the potential energy the state is called metastable. 
Remark. The validity of this expansion procedure is clearly called into question if any of the resonances $\sigma_{n}$ are very close to zero, in which case the complex frequency $\Lambda_{2}+\mathrm{i} \Gamma_{2}$ is potentially large. The breakdown of the expansion in this case indicates the presence of a parametric zero-energy resonance. Note, however, that in the odd case the matrix element $N^{(\mathrm{p})}(t, \lambda)$ vanishes as $\lambda$ tends to zero, and therefore so do the corresponding Fourier coefficients (and, in particular, they vanish to leading order in $\epsilon$, that is, $N_{1, n}(\lambda)$ vanishes at $\lambda=0$ for all $n)$. This suggests that the expansion (4.43) continues to hold in the odd case as the parameters $\rho_{1}$ and $\rho_{2}$ of the two-soliton potential are varied so as to cause a resonance $\sigma_{n}\left(\rho_{1}, \rho_{2}\right)$ to pass through zero. In the even case, however, behaviour possibly very different from that predicted by the formula (4.43) is expected if a resonance is close to zero. We plan to investigate this phenomenon analytically; however, in this paper we will demonstrate the effects of a parametric zero-energy resonance in both the even and odd cases with numerical simulations. Sudden changes in the behaviour of a simple model for atomic ionization as a parameter is smoothly varied, causing the system to pass through a zero-energy resonance, have recently been observed and compared with experiment by Costin et al [7].

\subsection{Rigorous analysis and infinite-time results}

The multiple-scale analysis of the preceding section leads to an asymptotic formula for the decaying bound state amplitude that is valid on time intervals of order $\epsilon^{-2}$. In this section, we will establish the validity of the asymptotic formula (4.43) in certain circumstances using the results of Kirr and Weinstein [15]. When applicable, these results also yield a detailed description of the solution as $t \rightarrow \pm \infty$.

More precisely, we now study the perturbed periodic system in the form obtained by use of Floquet factorization of the time-periodic unperturbed Hamiltonian $\mathcal{H}_{0}(t)$,

$$
\mathrm{i} \partial_{t} y-\mathcal{B} y=\tilde{\mathcal{W}}(t) y .
$$

The self-adjoint operator $\mathcal{B}: L^{2}(\mathbb{R}) \rightarrow L^{2}(\mathbb{R})$ defined in section 3 can be thought of as a timeindependent Hamiltonian, and the idea is to apply the theory of periodic (or almost periodic) perturbations of autonomous linear Hamiltonian systems as developed in $[15,28]$ directly to the problem in this form.

As we did in the multiple-scales analysis, we will restrict attention to the special case of periodically perturbed even two-soliton periodic potentials. As we know, in this case the operator $\mathcal{B}$ has exactly two $L^{2}$ eigenfunctions, one an even function of $x$ and the other an odd function of $x$. Since $L^{2}$ is the direct sum of its two subspaces $L_{(\mathrm{e}, \mathrm{o})}^{2}$ of even and odd functions, and since $\mathcal{B}$ leaves each subspace invariant, we may study the problem (4.44) restricted to one subspace at a time. This reduction results in an unperturbed problem with a single bound state, and to such problems the results described in $[15,28]$ can be applied without modification.

On each subspace $L_{(\mathrm{e}, \mathrm{o})}^{2}(\mathbb{R})$, the operator $\mathcal{B}$ is explicitly given by

$$
\begin{aligned}
\mathcal{B} f(x)=\left\langle\Psi_{\mathrm{b}}^{(\mathrm{e}, \mathrm{o})}(\cdot, 0),-2 \beta_{\mathrm{b}} f(\cdot)\right\rangle \Psi_{\mathrm{b}}^{(\mathrm{e}, \mathrm{o})}(x, 0) & \\
& +\int_{0}^{\infty}\left\langle\Psi_{\mathrm{d}}^{(\mathrm{e}, \mathrm{o})}(\cdot, 0, \lambda), 2 \lambda^{2} f(\cdot)\right\rangle \Psi_{\mathrm{d}}^{(\mathrm{e}, \mathrm{o})}(x, 0, \lambda) \mathrm{d} \lambda
\end{aligned}
$$

where the functions $\Psi_{\mathrm{b}}^{(\mathrm{e}, \mathrm{o})}(x, t)$ and $\Psi_{\mathrm{d}}^{(\mathrm{e}, \mathrm{o})}(x, t, \lambda)$ are defined given the parameters $\rho_{1}$ and $\rho_{2}$ in section 2. The hypotheses required in [15] of the even and odd restrictions of the operator $\mathcal{B}$ are reproduced here adapted to our application.

(H1) The even and odd restrictions of $\mathcal{B}$ are densely defined on subspaces of $L_{(\mathrm{e}, \mathrm{o})}^{2}(\mathbb{R})$ and have self-adjoint extensions to all of $L_{(\mathrm{e}, \mathrm{o})}^{2}(\mathbb{R})$. 
(H2) The spectrum of $\mathcal{B}$ in each of $L_{(\mathrm{e}, \mathrm{o})}^{2}(\mathbb{R})$ consists of an absolutely continuous part $\sigma_{\text {cont }}^{(\mathrm{e}, \mathrm{o})}(\mathcal{B})=[0, \infty]$ with associated spectral projection $\mathcal{P}_{\mathrm{c}}^{(\mathrm{e}, \mathrm{o})}$ and a single isolated eigenvalue $\lambda_{0}=-2 \beta_{\mathrm{b}}$ with corresponding normalized eigenstate $\psi_{0}(x)=\Psi_{\mathrm{b}}^{(\mathrm{e}, \mathrm{o})}(x, 0)$, so that

$$
\mathcal{B} \psi_{0}=\lambda_{0} \psi_{0} \quad\left\|\psi_{0}\right\|_{2}=1
$$

(H3) The odd restriction of $\mathcal{B}$ satisfies two dispersive local decay estimates. There exist constants $C_{\mathrm{ns}}$ and $C_{\mathrm{s}}$ such that

(a) The non-singular local decay estimate

$$
\left\|\langle\cdot\rangle^{-7 / 2} \mathrm{e}^{-\mathrm{i} B t} \mathcal{P}_{\mathrm{c}}^{(\mathrm{o})} f\right\|_{2} \leqslant C_{\mathrm{ns}}\langle t\rangle^{-3 / 2}\left\|\langle\cdot\rangle^{7 / 2} f\right\|_{2}
$$

holds for all $f \in L_{(\mathrm{o})}^{2}(\mathbb{R})$.

(b) The singular local decay estimate

$$
\left\|\langle\cdot\rangle^{-7 / 2} \mathrm{e}^{-\mathrm{i} \mathcal{B} t}(\mathcal{B}-2 \mu-2 \mathrm{i} \kappa 0)^{-1} \mathcal{P}_{\mathrm{c}}^{(\mathrm{o})} f\right\|_{2} \leqslant C_{\mathrm{s}}\langle t\rangle^{-3 / 2}\left\|\langle\cdot\rangle^{7 / 2} f\right\|_{2}
$$

where $\kappa=\operatorname{sgn}(t)$, holds uniformly for all $\mu$ satisfying $|\mu| \geqslant \mu_{\min }>0$, that is, the constant $C_{\mathrm{s}}$ only depends on $\mu_{\min }$.

The local decay hypotheses are established in appendix B. We remark here that due to a zeroenergy resonance, the decay estimates that are established in appendix B for the even case are of the form (4.47) and (4.48) but with decay rate $\langle t\rangle^{-1 / 2}$ (this is a sharp estimate). Unfortunately, this slower rate of decay precludes the direct application of the results in [15, 28]. On the other hand, as long as the perturbation does not create a resonance $\mu$ that is close to zero, we can expect similar results to hold in the even case over time scales of length $|t|<K / \epsilon^{2}$, since there is no obvious difficulty with the multiple-scales analysis.

The application of the results of $[15,28]$ also requires some hypotheses to be satisfied by the perturbation operator $\tilde{\mathcal{W}}(t)$ and its relation to the unperturbed Hamiltonian $\mathcal{B}$. The perturbation operator acting on $L_{(\mathrm{e}, \mathrm{o})}^{2}(\mathbb{R})$ takes the form

$$
\begin{aligned}
\tilde{\mathcal{W}}(t) f(x)= & M(t)\left\langle\Psi_{\mathrm{b}}^{(\mathrm{e}, \mathrm{o})}(\cdot, 0), f(\cdot)\right\rangle \\
& \left.+\int_{0}^{\infty} N^{(\mathrm{p})}(t, \eta)\left\langle\Psi_{\mathrm{d}}^{(\mathrm{e}, \mathrm{o})}(\cdot, 0, \eta), f(\cdot)\right\rangle \mathrm{d} \eta\right) \Psi_{\mathrm{b}}^{(\mathrm{e}, \mathrm{o})}(x, 0) \\
& +\int_{0}^{\infty}\left(N^{(\mathrm{p})}(t, \lambda)^{*}\left\langle\Psi_{\mathrm{b}}^{(\mathrm{e}, \mathrm{o})}(\cdot, 0), f(\cdot)\right\rangle\right. \\
& \left.+\int_{0}^{\infty} K^{(\mathrm{p})}(t, \lambda, \eta)\left\langle\Psi_{\mathrm{d}}^{(\mathrm{e}, \mathrm{o})}(\cdot, 0, \eta), f(\cdot)\right\rangle \mathrm{d} \eta\right) \Psi_{\mathrm{d}}^{(\mathrm{e}, \mathrm{o})}(x, 0, \lambda) \mathrm{d} \lambda .
\end{aligned}
$$

We recall that the periodic 'matrix elements' in the above expression are defined in terms of either the odd or even modes by (2.33). This operator, being periodic in $t$ with period $L$, has a Fourier series expansion

$$
\tilde{\mathcal{W}}(t)=\sum_{k=-\infty}^{\infty} \mathrm{e}^{2 \pi \mathrm{i} k t / L} \tilde{\mathcal{W}}_{k}
$$

where each operator $\tilde{\mathcal{W}}_{k}$ has the same form as (4.49) with the functions $M(t), N^{(\mathrm{p})}(t, \lambda)$ and $K^{(\mathrm{p})}(t, \lambda, \eta)$ replaced by the corresponding $k$ th Fourier coefficients, $M_{k}, N_{k}(\lambda)$ and $K_{k}(\lambda, \eta)$, respectively. The hypotheses required in [15] of the perturbation adapted to this context are: 
(H4) The operators $\tilde{\mathcal{W}}_{k}$ satisfy $\tilde{\mathcal{W}}_{-k}=\tilde{\mathcal{W}}_{k}^{\dagger}$ and

$$
\sum_{k=-\infty}^{\infty}\left\|\tilde{\mathcal{W}}_{k}\right\|_{\mathcal{L}\left(L^{2}(\mathbb{R})\right)}<\infty
$$

where $\|\cdot\|_{\mathcal{L}\left(L^{2}(\mathbb{R})\right)}$ denotes the uniform operator norm in $L^{2}(\mathbb{R})$. Also,

$$
|\|\tilde{\mathcal{W}}(\cdot)\|| \doteq \sum_{k=-\infty}^{\infty}\left(\left\|\langle\cdot\rangle^{7 / 2} \tilde{\mathcal{W}}_{k}\right\|_{\mathcal{L}\left(L^{2}(\mathbb{R})\right)}+\left\|\langle\cdot\rangle^{7 / 2} \tilde{\mathcal{W}}_{k}\langle\cdot\rangle^{7 / 2}\right\|_{\mathcal{L}\left(L^{2}(\mathbb{R})\right)}\right)<\infty
$$

(H5) The following resonance condition holds:

$$
\Gamma \doteq \pi \sum_{n=n_{0}}^{\infty}\left\langle\tilde{\mathcal{W}}_{n} \psi_{0}, \delta\left(\mathcal{B}-2 \sigma_{n}\right) \tilde{\mathcal{W}}_{n} \psi_{0}\right\rangle>0
$$

where the resonances are defined by $\sigma_{n}=\left(\lambda_{0}+2 \pi n / L\right) / 2=-\beta_{\mathrm{b}}+n \pi / L$. Here $n_{0}$ is the smallest positive integer for which $\sigma_{n_{0}}>0$. Note that since

$$
\tilde{\mathcal{W}}_{n} \psi_{0}=\tilde{\mathcal{W}}_{n} \Psi_{\mathrm{b}}^{(\mathrm{e}, \mathrm{o})}(x, 0)=M_{n} \Psi_{\mathrm{b}}^{(\mathrm{e}, \mathrm{o})}(x, 0)+\int_{0}^{\infty} N_{n}(\lambda)^{*} \Psi_{\mathrm{d}}^{(\mathrm{e}, \mathrm{o})}(x, 0, \lambda) \mathrm{d} \lambda
$$

and since for $\sigma>0$

$$
\langle f(\cdot), \delta(\mathcal{B}-2 \sigma) f(\cdot)\rangle=\int_{0}^{\infty}\left|\left(\mathcal{V} \mathcal{P}_{\mathrm{c}}^{(\mathrm{e}, \mathrm{o})} f\right)(\lambda)\right|^{2} \delta\left(2 \lambda^{2}-2 \sigma\right) \mathrm{d} \lambda=\frac{\left|\left(\mathcal{V} \mathcal{P}_{\mathrm{c}}^{(\mathrm{e}, \mathrm{o})} f\right)(\sqrt{\sigma})\right|^{2}}{4 \sqrt{\sigma}}
$$

the formula for $\Gamma$ can be written as

$$
\Gamma=\frac{\pi}{4} \sum_{n=n_{0}}^{\infty} \frac{N_{n}\left(\sqrt{\sigma_{n}}\right)}{\sqrt{\sigma_{n}}} .
$$

Note that if $N_{n}(\lambda)$ has an expansion in a small parameter $\epsilon$, with its leading term linear in $\epsilon$ as was assumed in the multiple-scale analysis, then the leading term of the corresponding expansion for $\Gamma(\epsilon)$ is exactly $\epsilon^{2} \Gamma_{2}$, where $\Gamma_{2}$ is correctly obtained by the multiple-scale analysis and is given by (4.36). The constant $\Gamma$ is a decay rate associated with the bound state of the unperturbed system. The statement that expression (4.53) should be positive for decay to occur as a consequence of resonant coupling to the continuum is attributed to Fermi and is known as 'Fermi's golden rule'. Again, because the decay constant $\Gamma$ is quadratic in the size of the perturbation, the exponential decay process is very slow for small perturbations. Thus, in the presence of a small perturbation $W(x, t)$, the bound state is said to be metastable.

(H6) There are no finite accumulation points of the resonances $\sigma_{n}, n \geqslant n_{0}$. This is satisfied automatically because the Fourier expansion of $\tilde{\mathcal{W}}(t)$ is that of a periodic function. The point here is that the results in [15] are more general; for example, this hypothesis is satisfied by finite Fourier sums with incommensurate frequencies. Yet further generalizations can be found in [15].

Verifying the hypothesis $(\mathrm{H} 4)$ would seem to require more detailed information about the correction to the potential energy $W(x, t)$ than we have used thus far. We merely point out at this time that by elementary Cauchy-Schwarz arguments applied to the unitarily equivalent operators $\mathcal{V} \tilde{\mathcal{W}}_{n} \mathcal{V}^{\dagger}$, one finds the estimate

$$
\left\|\tilde{\mathcal{W}}_{n}\right\|_{\mathcal{L}\left(L^{2}(\mathbb{R})\right)} \leqslant 2 \sqrt{\left|M_{k}\right|^{2}+2 \int_{0}^{\infty}\left|N_{n}(\lambda)\right|^{2} \mathrm{~d} \lambda+\int_{0}^{\infty} \int_{0}^{\infty}\left|K_{n}(\lambda, \eta)\right|^{2} \mathrm{~d} \lambda \mathrm{d} \eta .}
$$


Assuming these bounds are all finite, which is really a question of the smoothness and decay of 'snapshots' of the function $W(x, t)$ at fixed $t$, we see that the first required bound in (H4) will be satisfied if the Fourier coefficients of the function $W(x, t)$ in $t$ decay faster than, say, $1 / n$. This is because the other periodic contributions come from the analytic eigenfunctions, whose Fourier coefficients decay faster than $1 / n^{p}$ for any $p>0$. Therefore, not much beyond continuity in $t$ is required of $W(x, t)$, at least for this simpler estimate. More restrictions are certainly required to satisfy the second estimate of $(\mathrm{H} 4)$.

These hypotheses imply the following results:

Proposition 4.1 (theorem 2.1 of [15]). Let $\mathcal{B}$ and $\tilde{\mathcal{W}}(t)$ satisfy the above hypotheses and let an odd function $y_{0}(x)$ be given such that $\langle x\rangle^{7 / 2} y_{0}(x) \in L_{(\mathrm{o})}^{2}(\mathbb{R})$. Let $y(x, t)$ be the solution of (4.44) with initial condition $y(x, 0)=y_{0}(x)$. Then if $|\|\tilde{\mathcal{W}}(\cdot)\||$ is sufficiently small there exists a constant $C$ such that

$$
\left\|\langle\cdot\rangle^{-7 / 2} y(\cdot, t)\right\|_{2} \leqslant C\langle t\rangle^{-3 / 2}\left\|\langle\cdot\rangle^{7 / 2} y_{0}(\cdot)\right\|_{2}
$$

holds for all $t \in \mathbb{R}$.

Proposition 4.2 (theorem 2.2 of [15]). Assume the same hypotheses of $\mathcal{B}$ and $\tilde{\mathcal{W}}(t)$. Then if $|\|\tilde{\mathcal{W}}(\cdot)\||$ is sufficiently small, the solution $y(x, t)$ of $(4.44)$ corresponding to the odd initial condition $y_{0}(x)$ with $\langle x\rangle^{7 / 2} y_{0}(x) \in L_{(\mathrm{o})}^{2}(\mathbb{R})$ is of the form

$$
\begin{gathered}
y(x, t)=\left[\left\langle\Psi_{\mathrm{b}}^{(\mathrm{o})}(\cdot, 0), y_{0}(\cdot)\right\rangle \mathrm{e}^{2 \mathrm{i} \beta_{\mathrm{b}} t} \mathrm{e}^{-\Gamma|t|} \mathrm{e}^{\mathrm{i} \Lambda t} \mathrm{e}^{-\mathrm{i} \int_{0}^{t} M(s) \mathrm{d} s} \mathrm{e}^{\mathrm{i} r_{1}(t)}+r_{2}(t)\right] \Psi_{\mathrm{b}}^{(\mathrm{o})}(x, 0) \\
+\left(\mathrm{e}^{-\mathrm{i} t \mathcal{B}} \mathcal{P}_{\mathrm{c}}^{(\mathrm{o})} y_{0}(\cdot)\right)(x, t)+\tilde{y}(x, t)
\end{gathered}
$$

where

$$
\Lambda \doteq \sum_{n=-\infty}^{\infty}\left\langle\tilde{\mathcal{W}}_{n} \psi_{0}, \operatorname{PV}\left(\mathcal{B}-2 \sigma_{n}\right)^{-1} \mathcal{P}_{\mathrm{c}}^{(\mathrm{o})} \tilde{\mathcal{W}}_{n} \psi_{0}\right\rangle
$$

and where

- the phase correction $r_{1}(t)$ is uniformly bounded and $\mathrm{O}\left(|\|\tilde{\mathcal{W}}(\cdot)\||^{2}\right)$;

- the bound state amplitude error $r_{2}(t)$ is $\mathrm{O}(|\|\tilde{\mathcal{W}}(\cdot)\||)$ uniformly for all $|t|<K / \Gamma$ for all fixed $K$ and decays for large time as $\mathrm{O}\left(\langle t\rangle^{-3 / 2}\right)$;

- the correction $\tilde{y}(x, t)$ is orthogonal to the bound state: $\left\langle\psi_{0}, \tilde{y}(\cdot, t)\right\rangle=0$ for all $t$, and satisfies the dispersive decay estimate $\left\|\langle\cdot\rangle^{-7 / 2} \tilde{y}(\cdot, t)\right\|_{2}=\mathrm{O}\left(\langle t\rangle^{-3 / 2}\right)$ for large $t$.

Remark. Propositions 4.1 and 4.2 would appear to say that all initial conditions decay exponentially and then algebraically. However, a more careful reading shows that it is possible for there to be a transient stage of growth, before the decay ultimately sets in. This is because the error terms, although small when the perturbation is small, are not uniformly small for all initial conditions $y_{0}(x)$ such that $\langle x\rangle^{7 / 2} y_{0}(x)$ ranges over the unit sphere in $L^{2}(\mathbb{R})$. So, for each fixed perturbation $W(x, t)$, no matter how small, it is possible to find an initial condition $y_{0}(x, t)$ that grows before it decays. This is achieved by the following thought experiment. Suppose the periodic perturbation $W(x, t)$ is fixed and even in time $t$. Now pick any initial condition $y_{0}(x)$ so that $\langle x\rangle^{7 / 2} y_{0}(x) \in L^{2}(\mathbb{R})$. Proposition 4.1 guarantees that after a sufficiently large number $N$ of periods, the size of the solution of (4.44) when measured in the weighted $L^{2}$ norm is as small as we please. Note that throughout this process, the solution continues to satisfy $\left\|\langle\cdot\rangle^{7 / 2} y(\cdot, t)\right\|_{2}<\infty$. So now, start again at $t=0$ with the new initial condition $y_{0}(x)=y(x, t=N L)^{*}$. Since the potential is real and even in time, integration of (4.44) with 
this new initial condition is, up to complex conjugation, equivalent to integration backwards in time from the time $t=N L$ with the initial condition $y(x, t=N L)$. So we know that for this very small initial condition, the weighted $L^{2}$ norm must first grow to an order one size at time $t=N L$ as the decay process transiently reverses itself, before ultimately giving way to decay over longer times. The existence of such solutions does not violate the statement of propositions 4.1 or 4.2 because if one keeps the same initial condition and then makes the perturbation smaller yet again, the connection with the time-reversed problem is lost for this initial condition, and decay occurs sooner.

By the same arguments applied in the above discussion of the decay constant $\Gamma$, it follows that there is an alternative formula for $\Lambda$ :

$$
\Lambda=\sum_{n=-\infty}^{n_{0}-1} \int_{0}^{\infty} \frac{\left|N_{n}(\sqrt{\sigma})\right|^{2} \mathrm{~d} \sigma}{4 \sqrt{\sigma}\left(\sigma-\sigma_{n}\right)}-\sum_{n=n_{0}}^{\infty} \mathrm{PV} \int_{0}^{\infty} \frac{\left|N_{n}(\sqrt{\sigma})\right|^{2} \mathrm{~d} \sigma}{4 \sqrt{\sigma}\left(\sigma-\sigma_{n}\right)}
$$

Again, if $N_{n}(\lambda)$ has an expansion in a small parameter $\epsilon$ of the form $N_{n}(\lambda)=\epsilon N_{1, n}(\lambda)+\mathrm{O}\left(\epsilon^{2}\right)$ then the leading term of $\Lambda$ is of the form $\epsilon^{2} \Lambda_{2}$, where $\Lambda_{2}$ as given by (4.35) was resolved by the multiple-scales analysis. This frequency shift associated with the decay of the bound state is the Lamb shift.

From these results, one recovers the true dynamics by setting $f(x, t)=(\mathcal{P}(t) y(\cdot, t))(x, t)$, where $\mathcal{P}(t)$ is the periodic operator that appeared in the Floquet factorization of the propagator $\mathcal{U}(t)$ for the periodic unperturbed Hamiltonian $\mathcal{H}_{0}(t)$. Since

$$
\begin{aligned}
\left(\mathcal{P}(t) \mathrm{e}^{2 \mathrm{i} \beta_{\mathrm{b}} t} \Psi_{\mathrm{b}}^{(\mathrm{o})}(\cdot, t)\right)(x, t) & =\left(\mathcal{P}(t) \mathrm{e}^{-\mathrm{i} t \mathcal{B}} \Psi_{\mathrm{b}}^{(\mathrm{o})}(\cdot, t)\right)(x, t) \\
& =\left(\mathcal{U}(t) \Psi_{\mathrm{b}}^{(\mathrm{o})}(\cdot, t)\right)(x, t)=\Psi_{\mathrm{b}}^{(\mathrm{o})}(x, t)
\end{aligned}
$$

it follows that the time-dependent projection of $f(x, t)$ onto the bound state Bloch function $\Psi_{\mathrm{b}}^{(\mathrm{o})}(x, t)$ is uniformly approximated by

$$
B_{\mathrm{b}}(t) \doteq\left\langle\Psi_{\mathrm{b}}^{(\mathrm{o})}(\cdot, t), f(\cdot, t)\right\rangle \sim\left\langle\Psi_{\mathrm{b}}^{(\mathrm{o})}(\cdot, 0), f(\cdot, 0)\right\rangle \mathrm{e}^{-\Gamma|t|} \mathrm{e}^{\mathrm{i} \Lambda t} \mathrm{e}^{-\mathrm{i} \int_{0}^{t} M(s) \mathrm{d} s} .
$$

For the system restricted to the odd part of $L^{2}(\mathbb{R})$, these theorems provide justification for the formal multiple-scales analysis carried out above, and more. They globally describe the decay process for all time, where the multiple-scales calculation only attempts to capture the dynamics over time scales of length $\Gamma^{-1}$. On the other hand, since the rate of free dispersive decay is not sufficient in the even case to apply this detailed theory, we must settle for the multiple-scale expansions.

\section{Applications in planar waveguide optics}

In this section, we present a physical application of the kinds of perturbed time-dependent Schrödinger equations we have been studying in detail. This will provide a concrete family of perturbations $W(x, t)$ that we can use in subsequent numerical experiments.

\subsection{Time-dependent Schrödinger equations in waveguide optics}

For completeness, we present here a brief derivation of the time-dependent Schrödinger equation as it occurs in the paraxial theory of monochromatic waveguide optics. Consider Maxwell's wave equation for the electric field vector $\vec{E}(\vec{x}, t)$ in a planar $(\vec{x}=(y, z))$ dielectric medium with isotropic, inhomogeneous linear susceptibility $\chi^{(1)}(\vec{x}, t)$,

$$
\Delta \vec{E}-\frac{1}{c^{2}} \vec{E}_{t t}-\nabla(\nabla \cdot \vec{E})=\frac{1}{c^{2}}\left[\chi^{(1)}(\vec{x}, t) * \vec{E}\right]_{t t} .
$$


Here, the asterisk indicates convolution in time. A Fourier transform (denoted with the operator $\mathcal{F}$ ) in $t$ with dual variable $\omega$ (the optical frequency) leads to

$$
\Delta \mathcal{F} \vec{E}-\nabla(\nabla \cdot \mathcal{F} \vec{E})+\frac{\omega^{2} n^{2}(y, z, \omega)}{c^{2}} \mathcal{F} \vec{E}=0
$$

where the refractive index $n$ is defined by $n^{2}(y, z, \omega) \doteq 1+\left(\mathcal{F} \chi^{(1)}\right)(y, z, \omega)$. We now assume that the inhomogeneity is weak, so that gradients of $\left(\mathcal{F} \chi^{(1)}\right)(y, z, \omega)$ are small. This implies that in the absence of any free charges, the approximate relation $\nabla \cdot \mathcal{F} \vec{E} \approx 0$ follows from the exact relation for the electric displacement $\nabla \cdot \mathcal{F} \vec{D}=0$. Neglecting the divergence term in (5.2), one may then choose any unit vector $\vec{e}$ and set $(\mathcal{F} \overrightarrow{\vec{E}})(y, z, \omega)=\phi(y, z, \omega) \vec{e}$, which gives the Helmholtz or scalar wave equation for $\phi$,

$$
\phi_{z z}+\phi_{y y}+\frac{\omega^{2} n^{2}(y, z, \omega)}{c^{2}} \phi=0 .
$$

In the design of integrated optical devices, the inhomogeneity in the refractive index is a localized modulation of a 'background index' $n_{0}(\omega)$. Choose a fixed length scale $L_{0}$ and non-dimensionalize by setting $z / L_{0}=\delta^{-2} Z$ and $y / L_{0}=\delta^{-1} Y$, where $\delta$ is a dimensionless parameter, and $Y$ and $Z$ are dimensionless coordinates. Setting $\phi(y, z)=$ $f(Y, Z) \exp \left(\mathrm{i} \beta Z / \delta^{2}\right)$, where $\beta=L_{0} \omega n_{0}(\omega) / c$ is also dimensionless, one arrives at

$2 \mathrm{i} \beta \delta^{-2} f_{Z}+f_{Z Z}+\delta^{-2} f_{Y Y}+\beta^{2} \delta^{-4}\left[\frac{n^{2}\left(Y L_{0} \delta^{-1}, Z L_{0} \delta^{-}, \omega\right)}{n_{0}^{2}(\omega)}-1\right] f=0$.

With the definition

$$
Q(Y, Z ; \omega) \doteq-\frac{1}{2 \delta^{2}}\left[\frac{n^{2}\left(Y L_{0} \delta^{-1}, Z L_{0} \delta^{-2}, \omega\right)}{n_{0}^{2}(\omega)}-1\right]
$$

we see that the formal limit of $\delta \downarrow 0$ with $\beta$ and $Q(Y, Z ; \omega)$ held fixed yields the paraxial wave equation

$$
\mathrm{i} \beta f_{Z}+\frac{1}{2} f_{Y Y}-\beta^{2} Q(Y, Z ; \omega) f=0 .
$$

The potential function $Q(Y, Z ; \omega)$ vanishes as the refractive index approaches its background value $n_{0}(\omega)$, say as $Y$ and $Z$ go off to infinity (at least in most directions). Given a fixed function $Q(Y, Z ; \omega)$, we see that the paraxial approximation made here $(\delta \downarrow 0)$ is valid if the modulation in the refractive index is weak, slowly varying and more slowly varying in the $z$-direction than in the $y$-direction. That is, a fixed function $Q(Y, Z ; \omega)$ provides an asymptotic description of a family of physical refractive index profiles parametrized by $\delta \ll 1$,

$$
n^{2}(y, z, \omega ; \delta)=n_{0}^{2}(\omega)-2 \delta^{2} n_{0}^{2}(\omega) Q\left(\delta y / L_{0}, \delta^{2} z / L_{0} ; \omega\right) .
$$

Note that these assumptions about the refractive index justify a posteriori our neglect of the term $\nabla(\nabla \cdot \mathcal{F} \vec{E})$ in the original wave equation, because in the limit $\delta \rightarrow 0$, gradients of $n^{2}(y, z, \omega ; \delta)$ necessarily vanish.

\subsection{Spectral properties of paraxial waveguides}

In optical waveguide theory, integration (numerical or otherwise) of the linear Schrödinger equation (5.6), also known as the beam propagation method, is one of the main tools for studying the optical properties of 'long' planar structures like gradual fibre tapers or channel waveguide junctions, in which backward reflecting waves can be neglected. In this connection, a common problem that arises is the description of the change in behaviour of a waveguiding 
structure as the optical frequency is varied in the neighbourhood of some frequency $\omega_{0}$. If the structure $n^{2}(y, z, \omega)$ is one that admits the paraxial approximation, we can use the theory described above as a model. In this case, it is convenient to choose the length scale $L_{0}$ so that at the frequency $\omega_{0}$ we have $\beta=1$. With this choice, we think of $\beta=\beta(\omega)$ as a function of frequency satisfying $\beta\left(\omega_{0}\right)=1$. With the function $Q(Y, Z ; \omega)$ chosen consistently, the problem becomes one of studying the dependence of solutions of (5.6) on the frequency parameter $\omega$ near $\omega_{0}$. With the change of variables $x=Y \sqrt{\beta(\omega)}$ and $t=Z$, equation (5.6) takes the form

$$
\mathrm{i} f_{t}=\left(-\frac{1}{2} \partial_{x}^{2}+V_{0}(x, t)\right) f+W(x, t) f
$$

where

$$
V_{0}(x, t)=Q\left(x, t ; \omega_{0}\right)
$$

and the correction to the potential is given by

$$
W(x, t)=\beta(\omega) Q(x / \sqrt{\beta(\omega)}, t ; \omega)-Q\left(x, t ; \omega_{0}\right) .
$$

Setting $\epsilon=\omega / \omega_{0}-1$, we see that $W(x, t)=W(x, t ; \epsilon)$ is uniformly small in $\epsilon$ if $Q(Y, Z ; \omega)$ is in the Schwartz space with respect to $Y$. We have the expansion

$$
W(x, t ; \epsilon)=\epsilon \omega_{0}\left[\beta^{\prime}\left(\omega_{0}\right)\left(1-\frac{1}{2} x \partial_{x}\right) Q\left(x, t ; \omega_{0}\right)+\partial_{\omega} Q\left(x, t ; \omega_{0}\right)\right]+\mathrm{O}\left(\epsilon^{2}\right)
$$

uniformly in $x$ and $t$. If the frequency range of interest is sufficiently small, then it is often a good approximation to consider the problem to be dispersionless, so that the refractive index $n(y, z, \omega)$ is independent of $\omega$. In this paper, we will accordingly consider the function $Q$ to be independent of $\omega$ in which case $Q(x, t ; \omega)=V_{0}(x, t)$ for all $\omega$ in the range of interest, and we can drop the corresponding term in (5.11).

Suppose now that we choose to study a refractive index profile $n^{2}(y, z)$ that is even in $y$ and periodic in $z$, such that after choosing a frequency $\omega_{0}$ and non-dimensionalizing, the function $V_{0}(x, t)$ is one of the separable potentials described in detail at the end of section 2. Over length scales where the paraxial approximation is valid, this periodically modulated channel waveguide will actually have two 'breather modes', approximately described by the bound states $\Psi_{\mathrm{b}}^{(\mathrm{e})}(x, t)$ and $\Psi_{\mathrm{b}}^{(\mathrm{o})}(x, t)$. The effect of not being fully in the paraxial limit (that is, $\delta$ is small but finite) is that the modes will very slowly attenuate as they propagate forward due to a small coupling to backward-propagating fields. This small attenuation occurs at all frequencies near $\omega=\omega_{0}$ in a way that can be quantified [23]. However, the profile $n^{2}(y, z)$ is very special in that at the frequency $\omega=\omega_{0}$ there is no coupling between the bound modes and any forward-propagating radiation modes. This additional coupling would indeed be present for 'typical' $z$-periodic waveguide profiles $n^{2}(y, z)$.

In fact, the theory developed in section 4 can be applied to the perturbed problem (5.8) because the unperturbed potential $V_{0}(x, t)$ and the perturbation $W(x, t ; \epsilon)$ are both even functions of $x$ that are periodic in $t$ with the same period $L$. This theory shows that the additional attenuation due to coupling to forward-propagating radiation, while completely suppressed at the frequency $\omega_{0}$, re-emerges upon detuning the frequency slightly from $\omega_{0}$. Suppose the waveguide is cleaved at $z=0$ and is illuminated at this face with a broadband source consisting of many frequencies $\omega$. After some distance all of the frequencies will have attenuated somewhat due to backscattering (weak non-paraxiality). However, all frequencies except $\omega_{0}$ will additionally decay by forward-propagating radiation damping. The waveguide will therefore preferentially 'pass' light of the frequency $\dagger \omega_{0}$. These effects were observed numerically in $[2,3]$.

$\dagger$ Actually, the 'background' attenuation due to non-paraxiality $(\delta \neq 0)$ decreases slightly with increasing frequency. When this effect is combined with the frequency-dependent decay calculated from the paraxial approximation, the preferred frequency for which the loss is minimal is detuned slightly upward [23] by an amount that is $\mathrm{O}\left(\delta^{2}\right)$. 
Note that from the point of view of optical waveguide theory, the periodicity of the index $n(x, z)$ in $z$ is an important feature, since it gives rise to an attenuated frequency response that is a symmetric function of frequency $\omega$ in the neighbourhood of $\omega_{0}$. Thus, attenuation occurs whether $\omega$ is less than or greater than the frequency $\omega_{0}$ of structural instability. In contrast, channel waveguides, where $n(y, z)$ is independent of $z$, also exhibit frequency-dependent structural instability at cut-off frequencies where the number of bound states changes. However, in such waveguides the number of bound states (guided modes) is always an increasing function of frequency [20], which implies that an input beam that matches onto a mode at its cut-off frequency $\omega_{0}$ will attenuate for $\omega$ slightly less than $\omega_{0}$ but will remain bound and thus give rise to a significant transmission for $\omega$ slightly greater than $\omega_{0}$. Thus, whereas channel waveguides with $z$-independent refractive index profiles can behave as 'high-pass' components, $z$-periodic waveguides that at frequency $\omega_{0}$ are modelled by separable potentials can behave as 'band-pass' components.

\section{Numerical simulations}

Here, we describe some numerical simulations we performed to verify the analytical predictions where we expect them to apply. We also would like to explore the behaviour of the perturbed system in parameter regimes where we expect zero-energy resonances (see the remark at the end of section 4.2) to prevent the theory from applying in its current form. For concreteness, we considered periodic perturbations of two problems, each associated with a particular twosoliton separable periodic potential. The particular perturbation we selected was exactly the type considered in section 5, namely, given a separable two-soliton periodic potential $V_{0}(x, t)$, we numerically integrated the equation

$$
\mathrm{i} \partial_{t} f+\frac{1}{2(1+\epsilon)} \partial_{x}^{2} f-(1+\epsilon) V_{0}(x, t) f=0
$$

for several small values of $\epsilon$. This problem differs from the type to which the theory developed above applies only by a rescaling of $x$; in particular, the time scale is unaffected.

Let us give some details about our numerical scheme. We used a Fourier split-step method with a local truncation error of $\mathrm{O}\left(\Delta t^{3}\right)$ [24]. The spatial domain $\left[x_{L}, x_{R}\right]$ of $[-80,80]$ in the 'non-zero-energy resonance' case and $[-40,40]$ in the 'zero-energy resonance' case (see below for more details about these two cases) was discretized into 1024 points. The scheme splits the Hamiltonian into two parts: $\mathcal{H}(t)=\mathcal{H}_{1}+\mathcal{H}_{2}(t)$, where

$$
\mathcal{H}_{1} \doteq-\frac{1}{2(1+\epsilon)} \partial_{x}^{2} \quad \mathcal{H}_{2}(t) \doteq(1+\epsilon) V_{0}(x, t) .
$$

Let $\mathcal{U}^{\epsilon}(t, s)$ denote the propagator associated with (6.1). Let $\mathcal{U}_{1}^{\epsilon}(t-s)$ and $\mathcal{U}_{2}^{\epsilon}(t, s)$ be those associated with $\mathcal{H}_{1}$ and $\mathcal{H}_{2}(t)$. Then, the numerical scheme approximates the true integration over a time step of size $\Delta t$ as follows:

$$
\mathcal{U}^{\epsilon}(t+\Delta t, t) \sim \mathcal{U}_{1}^{\epsilon}(\Delta t / 4) \mathcal{U}_{2}^{\epsilon}(t+3 \Delta t / 4, t+\Delta t / 4) \mathcal{U}_{1}^{\epsilon}(\Delta t / 4)
$$

which has an error of order $\Delta t^{3}$. It is easy to see that, after getting started with a quarter-step, and until finishing with a quarter-step, iterating this approximation to the propagator $\mathcal{U}^{\epsilon}(t, s)$ over many steps amounts to simply alternating between $\mathcal{U}_{1}^{\epsilon}$ and $\mathcal{U}_{2}^{\epsilon}$ each acting over a half-step of length $\Delta t / 2$.

So, in each half-step, only one of the two parts is integrated. The half-step involving $\mathcal{H}_{1}$ is carried out in the Fourier transform domain where one multiplies by the explicit exponential of the operator. This step is thus exact in time, so that the only error appears in discretizing 


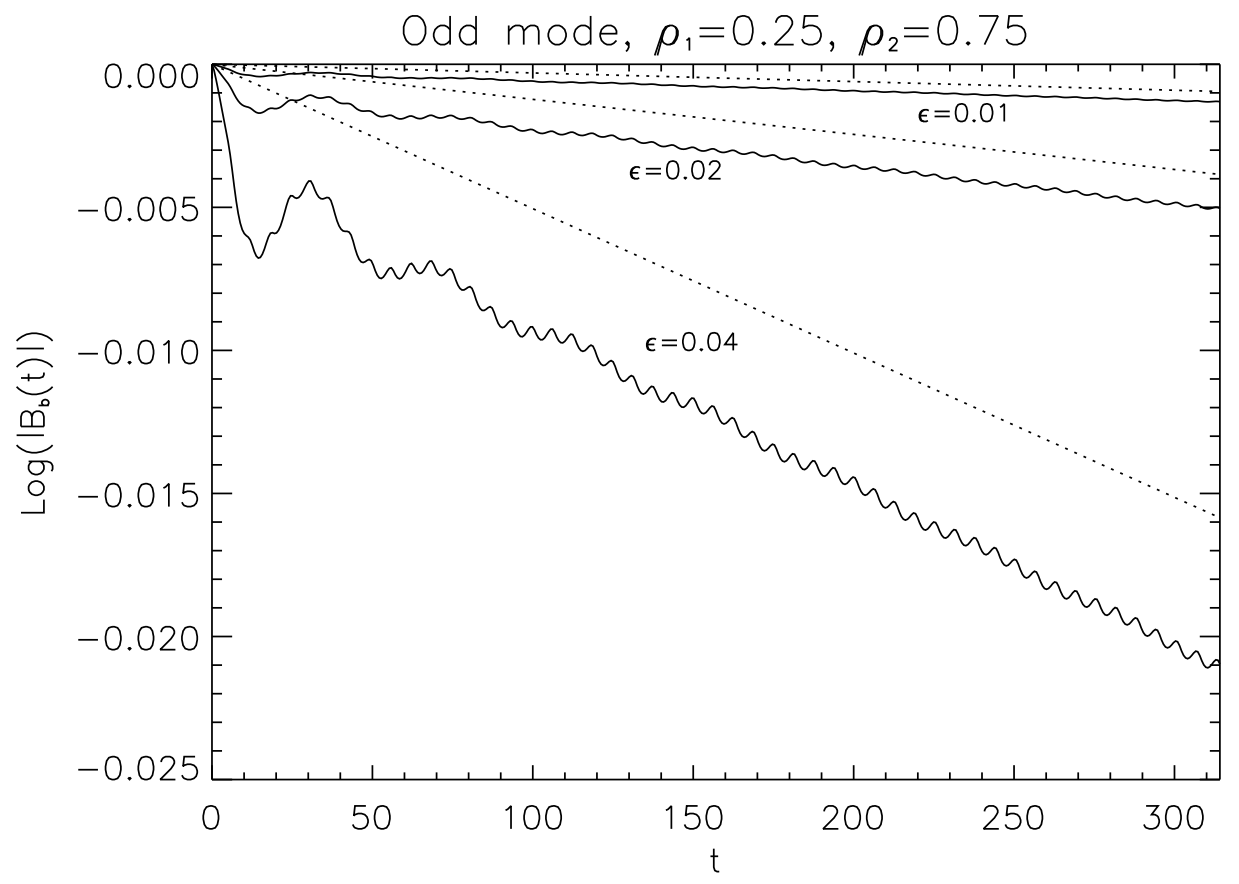

Figure 6. The magnitude of the projection of the solution onto the bound state. Odd mode. $\rho_{1}=\frac{1}{4}$, $\rho_{2}=\frac{3}{4}$. Full curves are the output from numerical simulations. Dotted lines are the analytical predictions.

the Fourier transform and is smaller than any power of $\Delta x$ if the functions to be differentiated are taken to be arbitrarily smooth. The half-step involving $\mathcal{H}_{2}(t)$ is done exactly because we have explicit formulae for $V_{0}(x, t)$ and it is possible to find an explicit exponential of $\mathcal{H}_{2}(t)$. That is, we can write down a formula for the multiplication operator

$$
\mathcal{U}_{2}^{\epsilon}\left(t, t_{0}\right)=\exp \left(\mathrm{i}(1+\epsilon) \int_{t_{0}}^{t} V_{0}(x, s) \mathrm{d} s\right)
$$

and use it in the code. Since the temporal gradients of $V_{0}(x, t)$ can be large in some parts of each period and small in others, we adjusted the time step throughout the period.

We expect the perturbation to generate radiation from the central bound region of the potential, and we need to remove this radiation from the problem as it moves to large $|x|$. To take care of this we used a 'sponge layer' in which we effectively add a term of the form

$$
-\mathrm{i} d \cdot\left[\exp \left(-\left(\frac{x-x_{R}}{w}\right)^{2}\right)+\exp \left(-\left(\frac{x-x_{L}}{w}\right)^{2}\right)\right] f
$$

to the right-hand side of (6.1) for a positive damping factor $d$ and width $w$. These parameters were adjusted heuristically until it was observed, roughly speaking, that no energy was being artificially drawn out of the centre and that no energy that was radiated outward was either reflected or transmitted through to the other side of the periodic domain.

We integrated for 50 periods. In all the experiments it was arranged that the fundamental period was $L=2 \pi$. We initialized the field $f$ at $t=0$ to be a snapshot of either the odd or the even mode of the unperturbed problem. Then, after integrating, we calculated the projection 


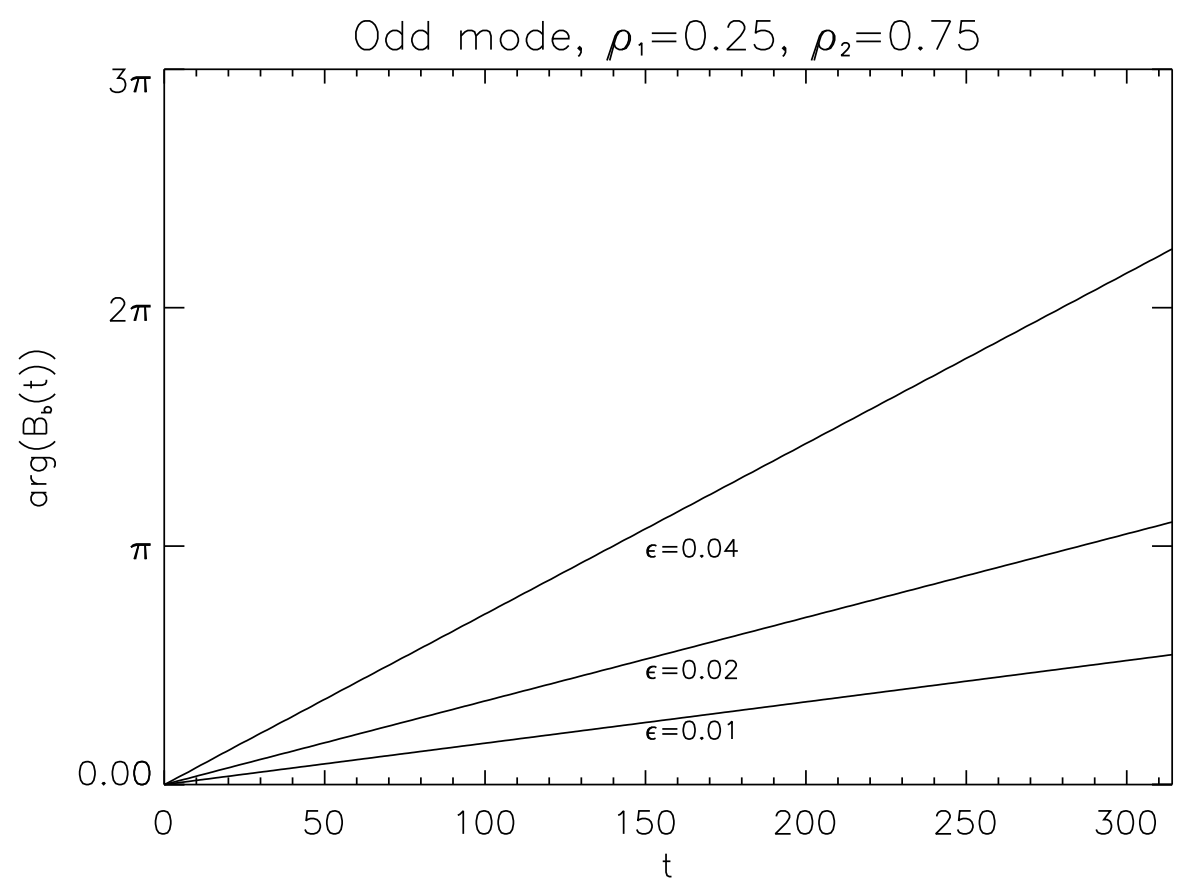

Figure 7. The phase of the projection of the solution onto the bound state. Odd mode. $\rho_{1}=\frac{1}{4}$, $\rho_{2}=\frac{3}{4}$.

of the numerical solution onto the exact solution of the unperturbed problem, defining

$$
B_{\mathrm{b}}(t) \doteq\left\langle\Psi_{\mathrm{b}}^{(\mathrm{e}, \mathrm{o})}(\cdot, t), f(\cdot, t)\right\rangle .
$$

We verified the accuracy of the code by checking that for $\epsilon=0$ we had $B_{\mathrm{b}}(t) \equiv 1$ to several digits, even in the presence of the damping in the sponge layer. Note that the function $B_{\mathrm{b}}(t)$ is related to $A_{\mathrm{b}}(t)$ by the simple relation

$$
B_{\mathrm{b}}(t)=A_{\mathrm{b}}(t) \mathrm{e}^{-2 \mathrm{i} \beta_{\mathrm{b}} t} .
$$

\subsection{Away from parametric zero-energy resonance}

For the first experiments, we selected $\rho_{1}=\frac{1}{4}$ and $\rho_{2}=\frac{3}{4}$ as the parameters of the function $V_{0}(x, t)$. It is easy to check that the period is $L=2 \pi$, and that the Floquet exponent of both odd and even bound states may be taken to be $\beta_{\mathrm{b}}=\rho_{1}^{2}=\frac{1}{16}$. Therefore, the resonances are explicitly given by

$$
\sigma_{n}=\frac{1}{2} n-\frac{1}{16}
$$

none of which are equal to zero. This means that there is no parametric zero-energy resonance, although in the even case there still is a zero-energy resonance corresponding to insufficient dispersive decay. In this case, the formula for the decay constant $\Gamma$ makes sense for both odd and even parity. Furthermore, for odd parity, we have a proof that the asymptotic expansion obtained previously is indeed valid.

In figure 6 , we show plots of $\log \left(\left|B_{\mathrm{b}}(t)\right|\right)$ for $\epsilon=0.04,0.02$ and 0.01 for an initial condition of odd parity. The numerical results are plotted as full curves, and superimposed 


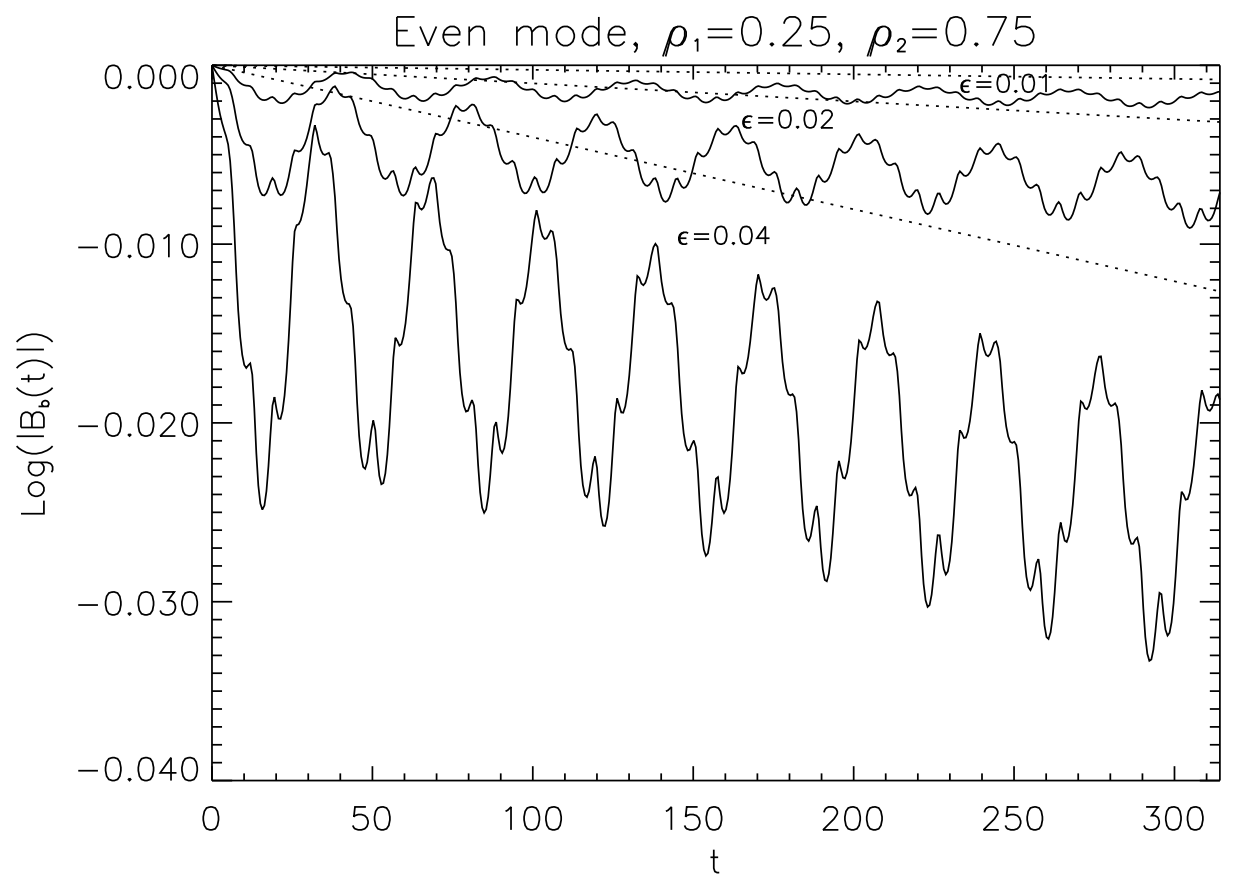

Figure 8. The magnitude of the projection of the solution onto the bound state. Even mode. $\rho_{1}=\frac{1}{4}, \rho_{2}=\frac{3}{4}$. Full curves are the output from numerical simulations. Dotted lines are the analytical predictions.

are corresponding graphs of $-\Gamma|t|$ calculated from the analytical formula, the analogue of Fermi's golden rule, and shown as dotted lines. The main observation here is that the graphs follow the corresponding straight lines, which have slopes that scale like $\epsilon^{2}$, as expected. The deviation from the straight lines appears to scale like $\epsilon^{2}$ as well, and to decay in time. In figure 7, we give corresponding plots of the argument of $B_{\mathrm{b}}(t)$ for an initial condition of odd parity. In these plots, it is easy to see that the phase grows roughly linearly in time, with a slope that is $\mathrm{O}(\epsilon)$. This is the contribution to the frequency shift of the term $\bar{M}$, which is indeed $\mathrm{O}(\epsilon)$.

Now, we consider an even initial condition, with corresponding projection $B_{\mathrm{b}}(t)$ onto the even mode of the exact solution for $\epsilon=0$. Figure 8 contains plots of $\log \left(\left|B_{\mathrm{b}}(t)\right|\right)$ as calculated from the numerical data for $\epsilon=0.04,0.02$ and 0.01 shown as full curves. Also plotted are the corresponding decay curves $-\Gamma|t|$ shown as dotted lines. Although for even parity there is insufficient dispersive decay for the results of [15] to apply, the decay constant $\Gamma$ (or more precisely as it is obtained in the multiple-scale analysis, $\epsilon^{2} \Gamma_{2}$ ) is finite because none of the resonances $\sigma_{n}$ are zero, and we see that the multiple-scale theory accurately predicts the rate of decay of the bound state even in this case. The plots of the phase of $B_{\mathrm{b}}(t)$ are shown in figure 9. Again, one sees that the rate of drift of the phase is $\mathrm{O}(\epsilon)$, as predicted by the multiple-scale theory.

The significant new feature apparently contributed by the lack of sufficient dispersive decay for initial conditions of even parity appears to be the quality of the deviations in $\left|B_{\mathrm{b}}(t)\right|$ from the 'backbone' decay $\mathrm{e}^{-\Gamma|t|}$. Not only are they larger for fixed $\epsilon$ than for initial conditions of odd parity, but they have an undulatory character that suggests a possible contribution of subharmonic frequencies to the dynamics. The period of the undulations superimposed on the 


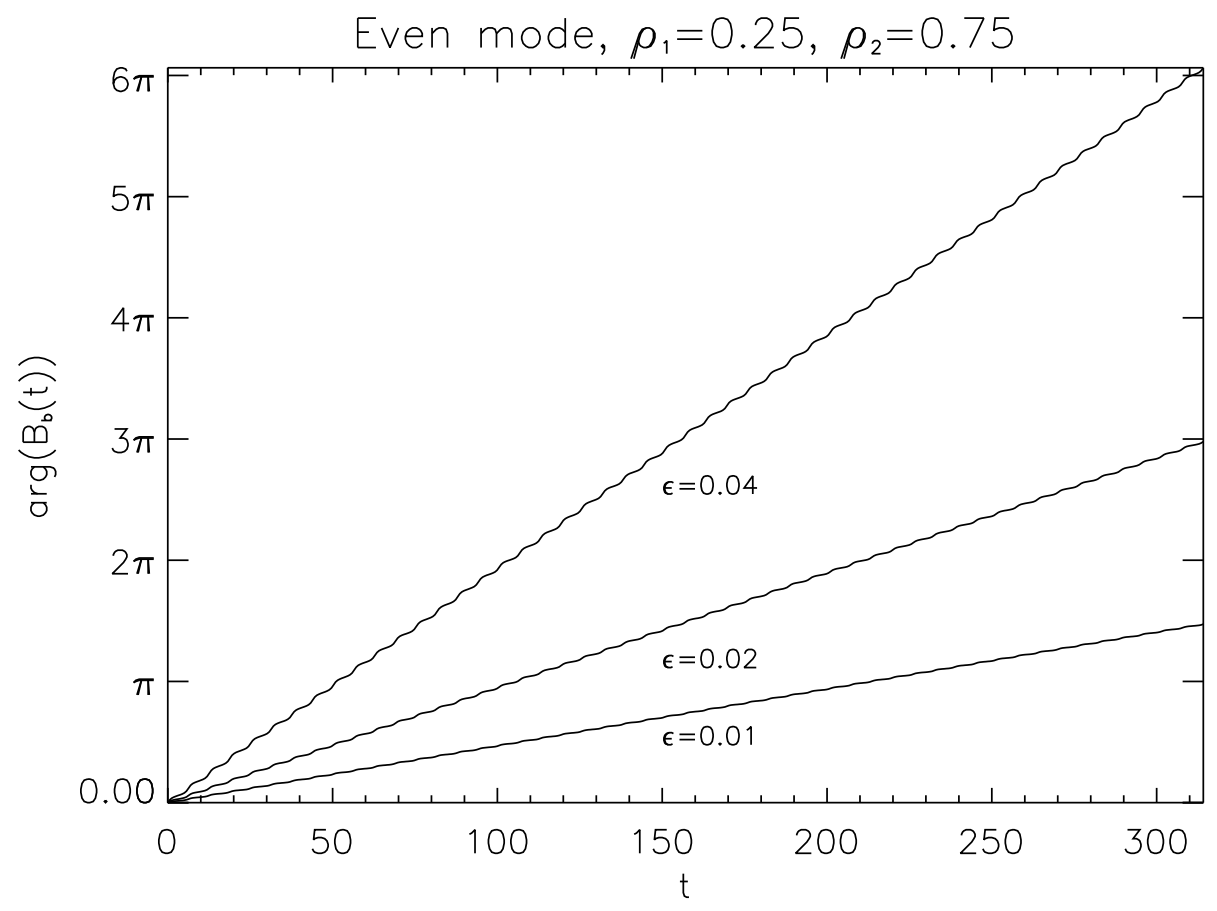

Figure 9. The phase of the projection of the solution onto the bound state. Even mode. $\rho_{1}=\frac{1}{4}$, $\rho_{2}=\frac{3}{4}$.

decay appears to be long compared with $L$, the fundamental period of the problem, but also appears to be more or less independent of $\epsilon$.

\subsection{At parametric zero-energy resonance}

As a second set of experiments, we considered a potential energy function $V_{0}(x, t)$ obtained from the parameters $\rho_{1}=1 / \sqrt{2}$ and $\rho_{2}=1$. In this case, the period is again $L=2 \pi$, and the Floquet exponents of both modes are $\beta_{\mathrm{b}}=\rho_{1}^{2}=\frac{1}{2}$. The corresponding family of resonances is

$$
\sigma_{n}=\frac{1}{2} n-\frac{1}{2} .
$$

One of these values is equal to zero. This condition for parametric zero-energy resonance always goes hand-in-hand with another property of this potential, namely that the Floquet multipliers of both modes are equal to 1 . Thus, both odd and even modes are actually periodic functions of $t$ with period $L$.

At a parametric zero-energy resonance, the dispersive local decay estimates fail to be sufficient to guarantee the applicability of the theory in [15], also for initial conditions of odd parity. However, in the odd case, the formulae for the decay constant $\Gamma$ and the Lamb shift $\Lambda$ are finite because there is sufficient vanishing in the numerator coming from the missing generalized eigenfunction at $\sigma=0$ to cancel and overcome the weaker vanishing of the denominator. Plots of $\log \left(\left|B_{\mathrm{b}}(t)\right|\right)$ for odd parity corresponding to $\epsilon=0.04,0.02$ and 0.01 are shown in figure 10 along with dotted lines indicating the analytical prediction of decay. The prediction of the theory appears to be very accurate indeed. The plots of the phase of $B_{\mathrm{b}}(t)$ 


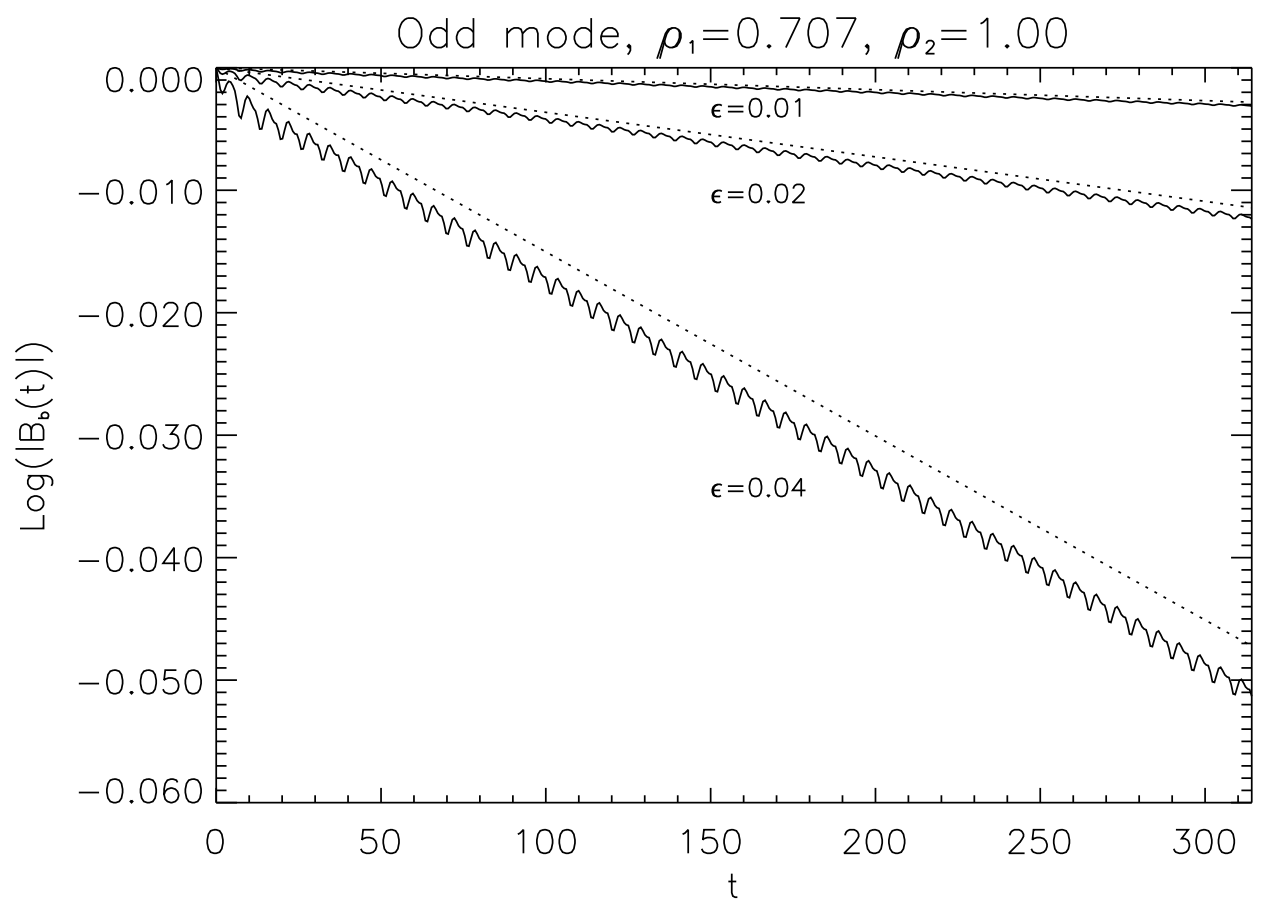

Figure 10. The magnitude of the projection of the solution onto the bound state. Odd mode. $\rho_{1}=1 / \sqrt{2}, \rho_{2}=1$. Full curves are the output from numerical simulations. Dotted lines are the analytical predictions.

are shown in figure 11. They show the frequency shift scaling like $\epsilon$, as we expect from the contribution of the term $\bar{M}$. So it appears that for initial conditions of odd parity, there is little if any effect of the parametric zero-energy resonance, although the rate of dispersive decay is smaller here than at more generic parameter values.

Finally, let us examine the behaviour of initial conditions of even parity. For such initial conditions and for these parameter values, we have both a simple zero-energy resonance (as one has in the even case for all parameter values) and a parametric zero-energy resonance (as occurs only for very special parameter values). It is easy to see that both $\Gamma$ and $\Lambda$ are infinite in this case, and clearly one cannot expect the multiple-scale analysis to be valid. So what can one expect? In figure 12 we plot $\log \left(\left|B_{\mathrm{b}}(t)\right|\right)$ for $\epsilon=0.04,0.02$ and 0.01 , as before. This time, rather than superimposing the straight lines $-\Gamma|t|$, we might try to compare with a 'renormalized' rate of decay given by the formula for $\Gamma$ with the term coming from $\sigma=0$ simply dropped. The straight lines calculated from the renormalized version of $\Gamma$ appear dotted on the plots. We still see quite good agreement at the level of a mean drift of $\left|B_{\mathrm{b}}(t)\right|$. As in the previous experiment with even parity, we see subharmonic undulations about this mean drift. However, a key point is that, whereas previously the period of these undulations appeared to be more or less independent of $\epsilon$, in this case we note that the period appears to scale like $\epsilon^{-1}$. Thus, there is a 'slow' dynamical process involving variations of the amplitude that is completely missed by the multiple-scales analysis in its current form. We must expect that whatever rescalings are required to balance the blowing up of $\Gamma$ in the vicinity of a parametric zero-energy resonance will also introduce interesting subharmonic dynamics on the scale of $T_{1}=\epsilon t$ that will reproduce the effects we are seeing numerically. As a final remark, the phase 


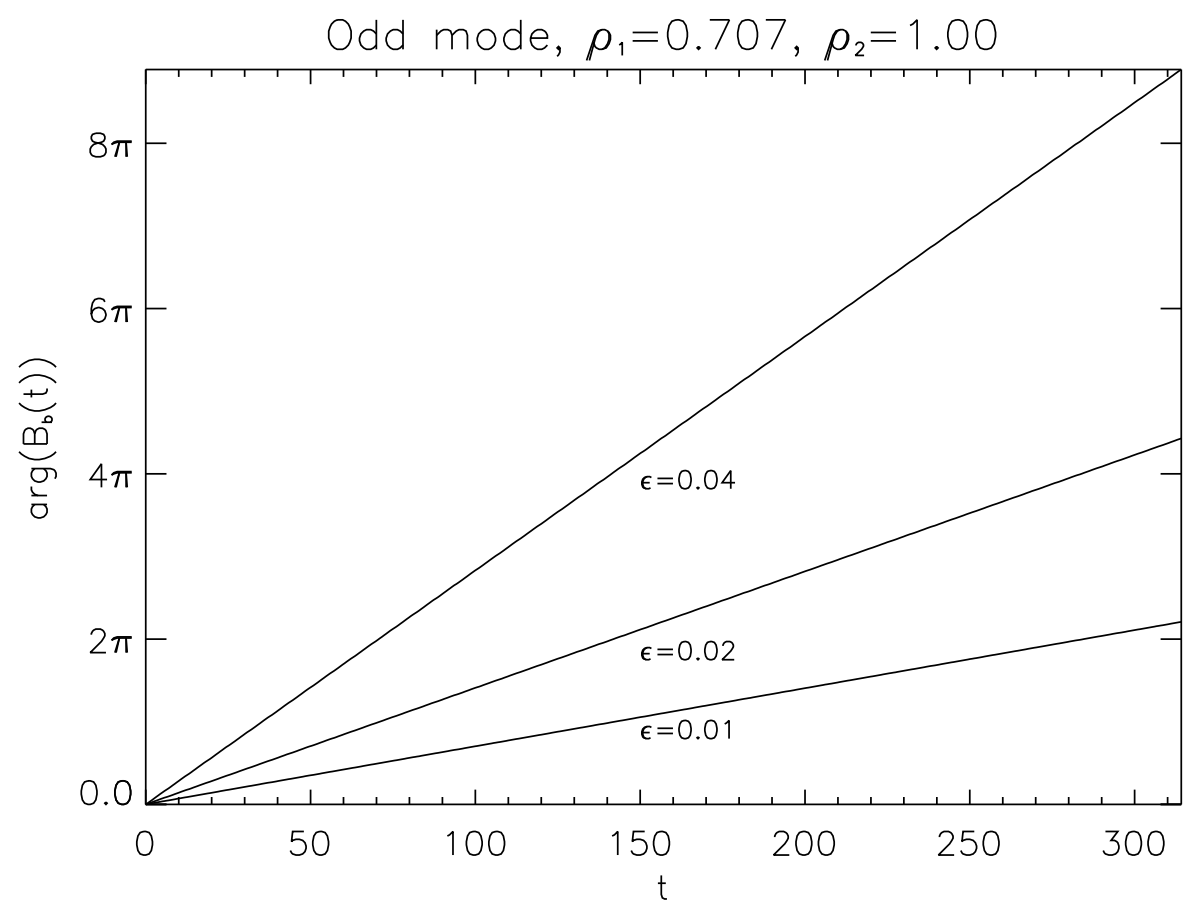

Figure 11. The phase of the projection of the solution onto the bound state. Odd mode. $\rho_{1}=1 / \sqrt{2}$, $\rho_{2}=1$.

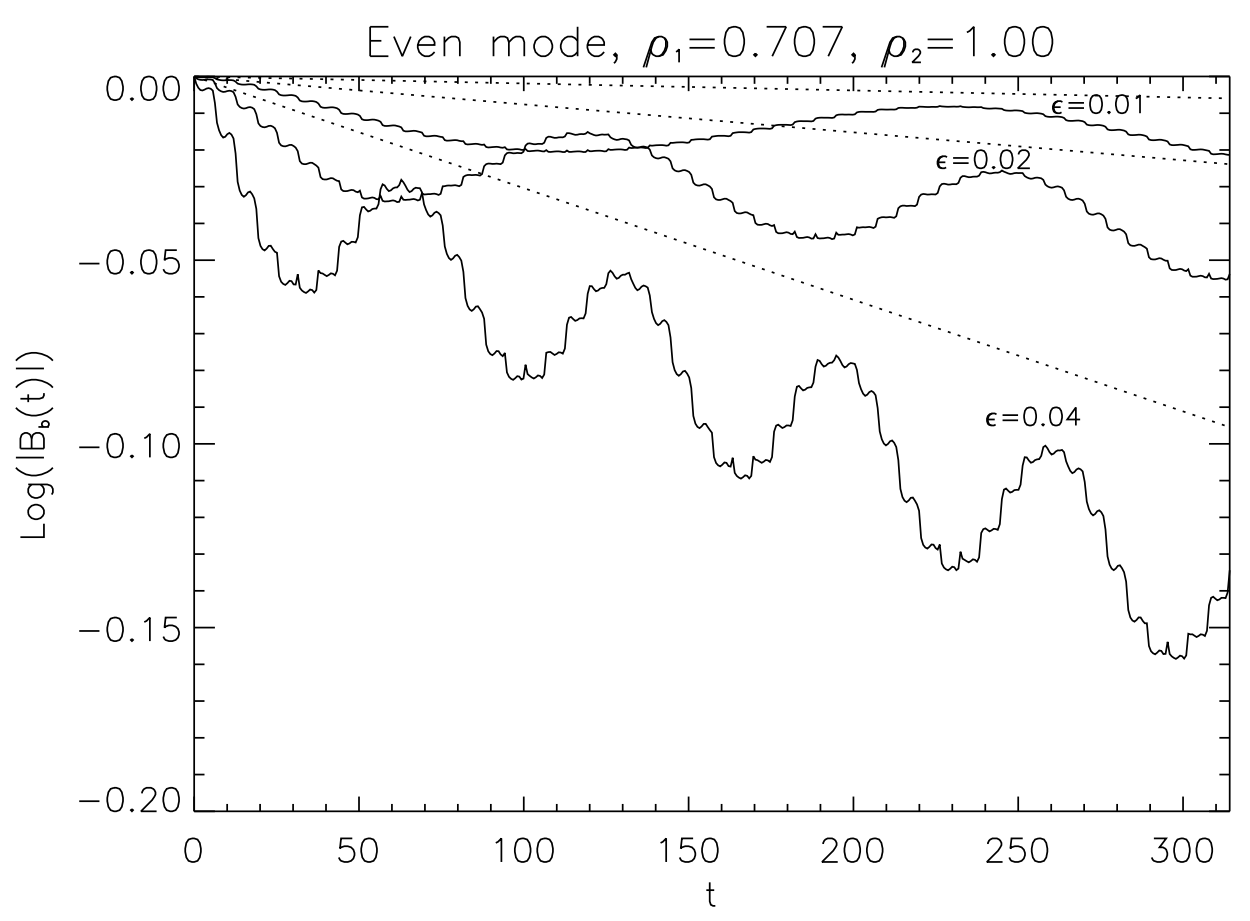

Figure 12. The magnitude of the projection of the solution onto the bound state. Even mode. $\rho_{1}=1 / \sqrt{2}, \rho_{2}=1$. Full curves are the output from numerical simulations. Dotted lines are the 'renormalized' analytical predictions. 


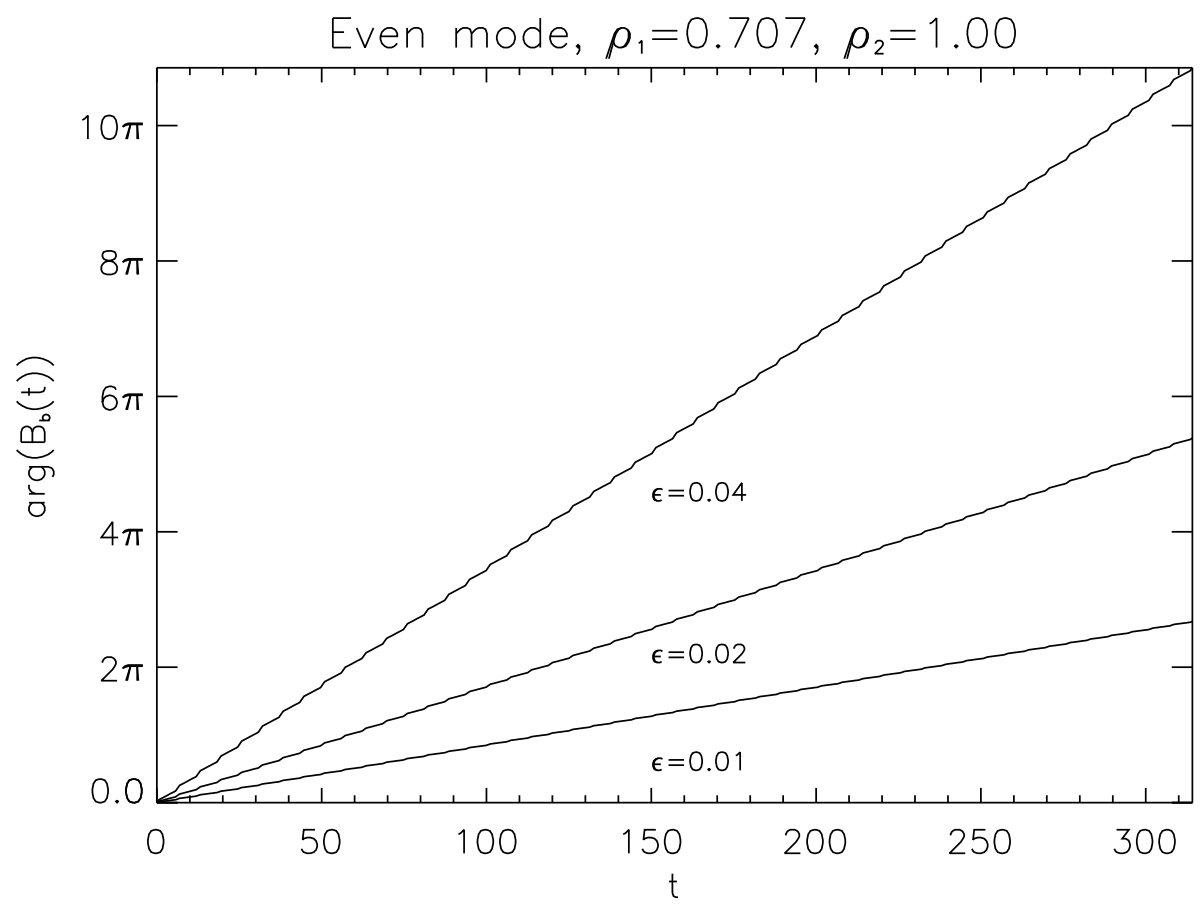

Figure 13. The phase of the projection of the solution onto the bound state. Even mode. $\rho_{1}=1 / \sqrt{2}, \rho_{2}=1$.

of $B_{\mathrm{b}}(t)$, as shown in figure 13, exhibits no particularly different behaviour than was seen in any of the other experiments. The frequency adjustment continues to be dominated by the relatively large term $\bar{M}$ and is therefore of order $\epsilon$.

\section{Conclusions}

In studying the propagation of waves in time-periodic potentials, considering the problem at hand to be a perturbation of a separable periodic problem is evidently as easy as, and in many cases more convenient than, working with periodic perturbations of stationary potential problems. A particular application to the theory of periodically modulated optical waveguides in planar dielectric media allows one to study frequency-dependent attenuation properties of certain optical waveguides.

Many of the difficulties described in our paper concern the influence of zero-energy resonances. These are generically not present (that is, for most separable periodic potentials, as for most stationary potentials), but are always present when the potential has sufficient symmetry, as in the evenness considered above. Many problems would therefore vanish upon dropping the symmetry. From one point of view, this introduces the additional complication of having multiple bound states that are essentially coupled to one another by the perturbation. The study of perturbed multimode problems arises naturally in the theory of light propagation in optical fibres having large effective cross sections. Some of the necessary modifications in the theory described in [15] are described by the same authors in [16].

Of course another point of view is to keep the symmetry, and hence the possibility of zero-energy resonance, and study the effect of the resonance in more detail. Our numerical 
experiments suggest that the effects of such a resonance are most dramatic when the expressions for $\Gamma$ and $\Lambda$ blow up, but we also see significant effects, presumably coming simply from the lack of a sufficiently long time decay for freely dispersing waves, when these quantities are finite. An asymptotic perturbation theory for small $\epsilon$ should be uniformly valid with respect to parameters such as $\rho_{1}$ and $\rho_{2}$, and we plan to investigate zero-energy resonances with such a goal in mind in future work.

\section{Acknowledgments}

P D Miller is grateful for the support of the NSF under grant number DMS 9304580 while at the Institute for Advanced Study. A Soffer is supported in part by a FAS-Rutgers grant and by NSF grant no DMS 9706780. M I Weinstein is supported in part by NSF grant no DMS 9500997. Collaboration began while M I Weinstein visited the IAS in March 1998 as part of the Programme in Geometric Partial Differential Equations organized by Karen Uhlenbeck, whom the authors thank for her support.

\section{Appendix A. Some theory of separable potentials}

For completeness, here we give a self-contained description of the separable potentials for the linear Schrödinger equation that are connected with the soliton theory of vector nonlinear Schrödinger equations. However, the material is auxiliary and all the needed facts are reproduced in the main text. The results here are not new [22] but some arguments are carried out in more detail.

Let $q_{1}(x, t), \ldots, q_{N}(x, t)$ be given smooth bounded complex functions of real $x$ and $t$, and let $A$ be the vector space of differentiable $\mathbb{C}^{N+1}$-valued functions of $x$ and $t$. Let $\lambda$ be a complex parameter, and consider the two linear operators acting in $A$

$$
\begin{aligned}
\mathcal{X}(\lambda, \vec{q}) & \doteq \partial_{x}-\left[\begin{array}{cc}
-2 \mathrm{i} \lambda & \vec{q}^{T} \\
-\vec{q}^{*} & \mathbf{0}
\end{array}\right] \\
& =\partial_{x}-(-2 \mathrm{i} \lambda \boldsymbol{E}+\boldsymbol{U}(\vec{q}))
\end{aligned}
$$

where $\boldsymbol{E}$ is a matrix whose elements are given by $E_{i j}=\delta_{i 1} \delta_{j 1}$ and

$$
\boldsymbol{U}(\vec{q})=\left[\begin{array}{cc}
0 & \vec{q}^{T} \\
-\vec{q}^{*} & \mathbf{0}
\end{array}\right]
$$

and

$$
\begin{aligned}
\mathcal{T}(\lambda, \vec{q}) & \doteq \partial_{t}-\left[\begin{array}{cc}
-2 \mathrm{i} \lambda^{2}+\mathrm{i} \vec{q}^{T} \vec{q}^{*} / 2 & \lambda \vec{q}^{T}+\mathrm{i} \partial_{x} \vec{q}^{T} / 2 \\
-\lambda \vec{q}^{*}+\mathrm{i} \partial_{x} \vec{q}^{*} / 2 & -\mathrm{i} \vec{q}^{*} \vec{q}^{T} / 2
\end{array}\right] \\
& =\partial_{t}-\left(-2 \mathrm{i} \lambda^{2} \boldsymbol{E}+\lambda \boldsymbol{U}(\vec{q})+\frac{1}{2} \mathrm{i} \boldsymbol{V}(\vec{q})\right)
\end{aligned}
$$

where

$$
\boldsymbol{V}(\vec{q})=\left[\begin{array}{cc}
\vec{q}^{T} \vec{q}^{*} & \partial_{x} \vec{q}^{T} \\
\partial_{x} \vec{q}^{*} & -\vec{q}^{*} \vec{q}^{T}
\end{array}\right] .
$$

Here $\vec{q}$ denotes the column vector of the functions $q_{k}(x, t)$ and $\mathbf{0}$ denotes the $N \times N$ zero matrix. Along with these two operators, we consider their nullspaces, $K_{\mathcal{X}}(\lambda, \vec{q}) \subset A$ and $K_{\mathcal{T}}(\lambda, \vec{q}) \subset A$. For generic $\lambda$, these subspaces are $(N+1)$-dimensional, and if restricted to generic fixed $x, t$ and $\lambda$ span $\mathbb{C}^{N+1}$. 
If the functions $q_{k}(x, t)$ are chosen just right, then the subspaces $K_{\mathcal{X}}(\lambda, \vec{q})$ and $K_{\mathcal{T}}(\lambda, \vec{q})$ may coincide for all complex $\lambda: K_{\mathcal{X}}=K_{\mathcal{T}} \doteq K$. If this is the case, then the common nullspace will certainly be contained in the nullspace of the commutator: $K \subset K_{[\mathcal{X}, \mathcal{T}]}$. As is easily checked, the commutator $[\mathcal{X}, \mathcal{T}]$ is not a differential operator, but is merely a matrix multiplication operator, with entries depending on $x$ and $t$ through the $q_{k}(x, t)$. Since the kernel of the commutator contains a subspace $K$ of dimension $N+1$ for most $\lambda, x$ and $t$, this implies that the operators $\mathcal{X}$ and $\mathcal{T}$ commute. It is easily checked that the compatibility condition $[\mathcal{X}, \mathcal{T}]=\mathbf{0}$ is equivalent to the vector nonlinear Schrödinger equation

$$
\mathrm{i} \partial_{t} \vec{q}+\frac{1}{2} \partial_{x}^{2} \vec{q}+\left(\vec{q}^{T} \vec{q}^{*}\right) \vec{q}=0 .
$$

It is therefore necessary that (A.5) be satisfied by the functions $q_{k}(x, t)$ if we are to have a basis of simultaneous nullvectors in the common nullspace $K$. When they exist, we can collect all these linearly independent column vectors into a square matrix $\boldsymbol{F}(x, t, \lambda)$. These ideas admit a natural geometric interpretation in the trivial frame bundle $E \rightarrow \mathbb{R}^{2}$ with fibre $G L(N+1, \mathbb{C})$. Here, $\mathcal{X}$ and $\mathcal{T}$ are covariant derivative operators for $E$ in the $x$ and $t$ directions, and the condition $[\mathcal{X}, \mathcal{T}]=\mathbf{0}$ means that the curvature of the affine connection specified by $\mathcal{X}$ and $\mathcal{T}$ is zero. This implies the existence of parallel global sections $\boldsymbol{F}(x, t, \lambda)$ of the bundle E.

Finding a global section $\boldsymbol{F}(x, t, \lambda)$ of $E$ given $\vec{q}(x, t)$ (that is, a matrix of simultaneous solution vectors) is not always easy and for this reason, we will adopt a different point of view below. However, it is clear from (A.1) and (A.3) that, given bounded functions $q_{k}(x, t)$ satisfying (A.5), it is possible to develop an asymptotic expansion for $\boldsymbol{F}(x, t, \lambda)$ in the limit $\lambda \rightarrow \infty$. The expansion may be sought in the form

$\boldsymbol{F}(x, t, \lambda)=\left(c \mathbb{I}_{N+1}+\lambda^{-1} \boldsymbol{F}^{(-1)}(x, t)+\lambda^{-2} \boldsymbol{F}^{(-2)}(x, t)+\cdots\right)\left[\begin{array}{cc}\mathrm{e}^{-2 \mathrm{i}\left(\lambda x+\lambda^{2} t\right)} & \overrightarrow{0}^{T} \\ \overrightarrow{0} & \mathbb{I}_{N}\end{array}\right]$.

Here, $\mathbb{I}_{D}$ denotes the $D \times D$ identity matrix and $c$ is a complex constant. The coefficient matrices $\boldsymbol{F}^{(p)}(x, t)$ are determined recursively in terms of $q_{1}(x, t), \ldots, q_{N}(x, t)$ and the constant $c$ by collecting powers of $\lambda$ in the compatible equations $\mathcal{X} \boldsymbol{F}=\mathcal{T} \boldsymbol{F}=0$. There is some ambiguity in this expansion procedure entering as integration constants at each order. However, it is easy to see that

$$
F_{1, k+1}^{(-1)}(x, t)=\frac{c}{2 \mathrm{i}} q_{k}(x, t) \quad k=1, \ldots, N
$$

regardless of the values of the integration constants.

The implications of this compatible structure for linear Schrödinger equations that are of interest to us in this paper are easily stated.

Proposition A.1. Suppose that (A.5) is satisfied, and let $\vec{v}(x, t, \lambda) \in K$ be any simultaneous nullvector of $\mathcal{X}(\lambda, \vec{q})$ and $\mathcal{T}(\lambda, \vec{q})$. Let $\mathcal{P}: A \rightarrow \mathbb{C}(x, t)$ be the operator of projection onto the first component. Define the self-consistent potential

$$
V_{0}(x, t) \doteq-\vec{q}(x, t)^{T} \vec{q}(x, t)^{*}
$$

and set $f(x, t, \lambda)=\mathcal{P} \vec{v}(x, t, \lambda)$. Then it follows that

$$
\mathrm{i} \partial_{t} f+\frac{1}{2} \partial_{x}^{2} f-V_{0}(x, t) f=0 .
$$

So, for each complex $\lambda$, the function $f(x, t, \lambda)$ is a solution of the linear, time-dependent Schrödinger equation with potential (A.8). Solutions corresponding to different values of $\lambda$ are linearly independent. Given functions $q_{k}(x, t)$ satisfying the nonlinear system (A.5), one 
can look to the common nullspace $K$ of the linear operators $\mathcal{X}(\lambda, \vec{q})$ and $\mathcal{T}(\lambda, \vec{q})$ as a source of many solutions of the linear equation (A.9).

Remark. Let us try to put these facts in a larger context, and incidentally give the proof of proposition A.1. It is part of the lore of integrable systems theory that linearized evolution equations connected with integrable systems are solvable in terms of 'squared eigenfunctions' coming from the auxiliary linear problems making up the Lax pair for the integrable system. The integrable system (A.5) is the compatibility condition for the equations $\mathcal{X} \boldsymbol{F}=\mathbf{0}$ and $\mathcal{T} \boldsymbol{F}=\mathbf{0}$. By a change of variables (gauge transformation) $\boldsymbol{F}=\boldsymbol{G} \exp \left(-\mathrm{i} \lambda x-\mathrm{i} \lambda^{2} t\right)$ the two equations take the more familiar form of the Lax pair for (A.5) $[9,19]$,

$$
\partial_{x} \boldsymbol{G}=\boldsymbol{A} \boldsymbol{G} \quad \partial_{t} \boldsymbol{G}=\boldsymbol{B} \boldsymbol{G}
$$

where

$\boldsymbol{A}=\left[\begin{array}{cc}-\mathrm{i} \lambda & \vec{q}^{T} \\ -\vec{q}^{*} & \mathrm{i} \lambda \mathbb{I}\end{array}\right] \quad \boldsymbol{B}=\left[\begin{array}{cc}-\mathrm{i} \lambda^{2}+\mathrm{i} \vec{q}^{T} \vec{q}^{*} / 2 & \lambda \vec{q}^{T}+\mathrm{i} \partial_{x} \vec{q}^{T} / 2 \\ -\lambda \vec{q}^{*}+\mathrm{i} \partial_{x} \vec{q}^{*} / 2 & \mathrm{i} \lambda^{2} \mathbb{I}-\mathrm{i} \vec{q}^{*} \vec{q}^{T} / 2\end{array}\right]$.

If $\boldsymbol{G}_{\alpha}$ and $\boldsymbol{G}_{\beta}$ are any two simultaneous matrix solutions of the Lax pair (A.10), and if $\boldsymbol{C}$ is any constant (that is, $x$ - and $t$-independent) matrix, then by setting $Q=G_{\alpha} C G_{\beta}^{-1}$, one easily obtains the equations

$$
\partial_{x} \boldsymbol{Q}=[\boldsymbol{A}, \boldsymbol{Q}] \quad \partial_{t} \boldsymbol{Q}=[\boldsymbol{B}, \boldsymbol{Q}] .
$$

Equations of this form are called Lax equations, and the elements of $\boldsymbol{Q}$ are the 'squared eigenfunctions'. The terminology becomes accurate in the scalar case $N=1$ when $\boldsymbol{A}$ and $\boldsymbol{B}$ are in the Lie algebra $s l(2)$. In this case the solutions $G$ of the Lax pair can be normalized to be in the Lie group $S L(2)$ and therefore have determinant one. Then, because $G_{\beta}$ is $2 \times 2$ with determinant one, the elements of $\boldsymbol{Q}$ are seen to be bona fide quadratic forms in the solutions of the Lax pair (A.10). The emphasis in the literature on the $s l(2)$-specific terminology of 'squared eigenfunctions' for the forms that satisfy the Lax equations (A.12) no doubt bears witness to the fact that so many of the famous integrable equations (e.g. Korteweg-de Vries, scalar nonlinear Schrödinger, sine-Gordon) are associated with $s l(2)$ representations.

If one introduces the splitting of a matrix into blocks: $M=M^{\mathrm{D}}+M^{\mathrm{OD}}$ where $M^{\mathrm{D}}$ consists of the $1 \times 1$ and $N \times N$ diagonal blocks of $M$ and $M^{\mathrm{OD}}$ consists of the $1 \times N$ and $N \times 1$ off-diagonal blocks of $\boldsymbol{M}$, and if one introduces $\boldsymbol{A}_{0}=\left.\boldsymbol{A}\right|_{\lambda=0}$ and $\boldsymbol{B}_{0}=\left.\boldsymbol{B}\right|_{\lambda=0}$, then it is an exercise to check that equations (A.12) imply

$$
\left[\begin{array}{cc}
\mathrm{i} & \overrightarrow{0}^{T} \\
\overrightarrow{0} & -\mathrm{i} \mathbb{I}
\end{array}\right] \partial_{t} \boldsymbol{Q}^{\mathrm{OD}}+\frac{1}{2} \partial_{x}^{2} \boldsymbol{Q}^{\mathrm{OD}}-\left[\begin{array}{cc}
\mathrm{i} & \overrightarrow{0}^{T} \\
\overrightarrow{0} & -\mathrm{i} \mathbb{I}
\end{array}\right]\left[\boldsymbol{B}_{0}^{\mathrm{D}}, \boldsymbol{Q}^{\mathrm{OD}}\right]-\frac{1}{2}\left[\boldsymbol{A}_{0}^{\mathrm{OD}},\left[\boldsymbol{A}_{0}^{\mathrm{OD}}, \boldsymbol{Q}^{\mathrm{OD}}\right]\right]=\mathbf{0} \text {. }
$$

If one writes

$$
Q^{\mathrm{OD}}=\left[\begin{array}{cc}
0 & \vec{g}^{T} \\
\vec{h} & \mathbf{0}
\end{array}\right]
$$

then one finds

$$
\begin{aligned}
& \mathrm{i} \partial_{t} \vec{g}^{T}+\frac{1}{2} \partial_{x}^{2} \vec{g}^{T}+\vec{q}^{T} \vec{q}^{*} \vec{g}^{T}+\vec{q}^{T} \vec{h} \vec{q}^{T}+\vec{g}^{T} \vec{q}^{*} \vec{q}^{T}=\overrightarrow{0} \\
& -\mathrm{i} \partial_{t} \vec{h}+\frac{1}{2} \partial_{x}^{2} \vec{h}+\vec{q}^{*} \vec{q}^{T} \vec{h}+\vec{q}^{*} \vec{g}^{T} \vec{q}^{*}+\vec{h}^{T} \vec{q}^{*}=\overrightarrow{0} .
\end{aligned}
$$

These linear equations for $\vec{g}$ and $\vec{h}$ are consistent with the constraint $\vec{h}=\vec{g}^{*}$ at which point it becomes clear that $\vec{g}(x, t)$ satisfies the linearization of the vector nonlinear Schrödinger equation (A.5) about a solution $\vec{q}(x, t)$. 
Consider now a particular solution $\vec{q}(x, t)$ of (A.5) and by adjoining a new trivial component $q_{N+1}(x, t) \equiv 0$, view it as a solution $\vec{q}^{\prime}(x, t)$ of (A.5) in the $(N+1)$-component case. From (A.15) it is easily seen that the corresponding components $g_{N+1}(x, t)$ and $h_{N+1}(x, t)$ satisfy

$$
\begin{aligned}
& \mathrm{i} \partial_{t} g_{N+1}+\frac{1}{2} \partial_{x}^{2} g_{N+1}+\left(\vec{q}^{T} \vec{q}^{*}\right) g_{N+1}=0 \\
& -\mathrm{i} \partial_{t} h_{N+1}+\frac{1}{2} \partial_{x}^{2} h_{N+1}+\left(\vec{q}^{T} \vec{q}^{*}\right) h_{N+1}=0
\end{aligned}
$$

where we have used the fact that $\vec{q}^{T} \vec{q}^{\prime *}=\vec{q}^{T} \vec{q}^{*}$. Now considering the Lax pair (A.10) for the primed potentials, it is easy to see that there exists a non-trivial column vector solution of both equations of the form $\left(\overrightarrow{0}_{N+1}^{T}, \exp \left(\mathrm{i} \lambda x+\mathrm{i} \lambda^{2} t\right)\right)^{T}$, and that further column vector solutions can then be chosen to have a vanishing last component. Taking the last column of the matrix solution $\boldsymbol{G}_{\beta}$ to be this particular solution, and the first $N+1$ columns all to have zeros in the final component, we see that $G_{\beta}$ may be inverted into two independent blocks, and therefore a solution of the linearized equation is given by

$g_{N+1}(x, t)=Q_{1, N+2}(x, t, \lambda)=\exp \left(-\mathrm{i} \lambda x-\mathrm{i} \lambda^{2} t\right) \sum_{k=1}^{N+2} C_{k, N+2}(\lambda) G_{\alpha, 1, k}(x, t, \lambda)$.

Since the matrix $C$ is arbitrary, we may view the sum above as the first component of an arbitrary column vector solution of the Lax pair (A.10) with the primed potentials $\vec{q}^{\prime}(x, t)$. Moreover, since $q_{N+1}^{\prime}(x, t) \equiv 0$, the first component of a solution of the primed Lax pair is also the first component of a solution of the unprimed Lax pair for the fully non-trivial potential $\vec{q}(x, t)$. Reversing the gauge transformation between solutions $\boldsymbol{G}$ of the unprimed Lax pair (A.10) and solutions $\boldsymbol{F}$ of $\mathcal{X} \boldsymbol{F}=\mathcal{T} \boldsymbol{F}=\mathbf{0}$ then establishes the connection with proposition A.1.

So, the procedure we are using for solving the time-dependent linear Schrödinger equation is exactly the 'squared eigenfunction' linearization of a certain $N+1$ component nonlinear Schrödinger equation about a particular solution having $q_{N+1}^{\prime}(x, t) \equiv 0$. The 'squared eigenfunctions' solving the linearized problem appear to be linear in this special case because for $q_{N+1}^{\prime} \equiv 0$ the primed Lax pair becomes partly trivial, and the contribution of this trivial part to the matrix $\boldsymbol{Q}$ is completely explicit (the exponential function that we remove with a gauge transformation).

We now return to the construction of self-consistent potentials and the corresponding solutions of (A.9). The nonlinear equation (A.5) is an integrable system by virtue of its representation as the compatibility condition of two linear problems. So there are many well known ways to find functions $q_{k}(x, t)$ for which the corresponding linear Schrödinger equation can be solved. However, as we are interested as much in the common nullspace of $\mathcal{X}$ and $\mathcal{T}$ as in the functions $q_{k}(x, t)$, we will now describe an effective approach to finding both at the same time. In this approach, the object of fundamental importance is the common nullspace $K$ itself. We construct it first, with the functions $q_{k}(x, t)$ being chosen after the fact precisely so that for any basis matrix $\boldsymbol{F}$ of $K$, we will have $\mathcal{X}(\lambda, \vec{q}) \boldsymbol{F}=\mathcal{T}(\lambda, \vec{q}) \boldsymbol{F}=\mathbf{0}$.

What we know about $K$ is that whenever it exists by virtue of the compatibility condition, the assumption that the functions $q_{k}(x, t)$ are bounded (this will be justified below) leads to expansions for large $\lambda$ of a basis for $K$ of the form (A.6). These expansions are generally only asymptotic; there is no guarantee that there exists a choice of the integration constants such that the expansion (A.6) converges for any $\lambda$ at all. However, we now suppose that there exist solutions $q_{k}(x, t)$ of the nonlinear system (A.5) for which an expansion (A.6) not only converges in some deleted neighbourhood of $\lambda=\infty$, but actually truncates. For such 
solutions $q_{k}(x, t)$, if they exist, a basis of the subspace $K$ is given exactly by an expression of the form

$$
\boldsymbol{F}(x, t, \lambda)=\left(c \lambda^{M} \mathbb{I}_{N+1}+\sum_{p=0}^{M-1} \lambda^{p} \boldsymbol{F}^{(p)}(x, t)\right)\left[\begin{array}{cc}
\mathrm{e}^{-2 \mathrm{i}\left(\lambda x+\lambda^{2} t\right)} & \overrightarrow{0}^{T} \\
\overrightarrow{0} & \mathbb{I}_{N}
\end{array}\right]
$$

for some positive integer $M$, where $c$ is a complex constant. We have multiplied by an explicit factor of $\lambda^{M}$ to bring the sum into polynomial form.

Since we are not considering the functions $q_{k}(x, t)$ to be known, we do not have the option of solving for the coefficient matrices $\boldsymbol{F}^{(p)}(x, t)$ by substitution into the equations $\mathcal{X} \boldsymbol{F}=\mathcal{T} \boldsymbol{F}=0$. We therefore must consider them to be arbitrary functions of $x$ and $t$ until we know otherwise. Without any constraints on the coefficients, we see that the differentiable matrix functions of $x, t$ and $\lambda$ of the form (A.18), for given integer values of $M$ and $N$, form a vector space $\Lambda_{N, M}$ over the complex numbers.

The space $\Lambda_{N, M}$ is very large. If our claim-that appropriate solutions $q_{k}(x, t)$ of the nonlinear system (A.5) exist-is not vacuous, then $\Lambda_{N, M}$ should contain many proper subspaces that may be identified with the common nullspace $K$ of $\mathcal{X}(\lambda, \vec{q})$ and $\mathcal{T}(\lambda, \vec{q})$ for some $\vec{q}$. If $\boldsymbol{F}(x, t, \lambda)$ is of the form (A.18) and is a basis matrix of one of these subspaces, then it must be determined modulo the constant $c$. This means that each such subspace of $\Lambda_{N, M}$ should ultimately be isomorphic to $\mathbb{C}$, with the isomorphism being established via the constant $c$.

We prepare to isolate the appropriate subspaces of $\Lambda_{N, M}$ by defining a set of discrete data. Let $\mathcal{D}$ denote an $M$-tuple of pairs $\left(\lambda_{k}, \vec{g}^{(k)}\right)$ where the $\lambda_{k}$ are distinct numbers in the complex upper half-plane and where the $\vec{g}^{(k)}$ are vectors in $\mathbb{C}^{N}$. From each vector $\vec{g}^{(k)}$, we build $N+1$ vectors in $\mathbb{C}^{N+1}$ by setting

$$
\vec{a}^{(k)}=\left(-1, g_{1}^{(k) *}, g_{2}^{(k) *}, \ldots, g_{N}^{(k) *}\right)^{T} \in \mathbb{C}^{N+1}
$$

and for $j=1, \ldots, N$,

$$
\vec{b}^{(k, j)}=\left(g_{j}^{(k)}, \vec{e}_{j}^{T}\right)^{T} \in \mathbb{C}^{N+1}
$$

where $\vec{e}_{j}$ are the usual unit vectors in $\mathbb{C}^{N}$.

Definition A.1. $\Lambda_{N, M}^{\mathcal{D}}$ is the subspace of $\Lambda_{N, M}$ whose elements $\boldsymbol{F}(x, t, \lambda)$ satisfy the homogeneous linear conditions

$$
\boldsymbol{F}\left(x, t, \lambda_{k}\right) \vec{a}^{(k)}=\overrightarrow{0}
$$

for $k=1, \ldots, M$ and

$$
\boldsymbol{F}\left(x, t, \lambda_{k}^{*}\right) \vec{b}^{(k, j)}=\overrightarrow{0}
$$

for $k=1, \ldots, M$ and $j=1, \ldots, N$.

It is not hard to use dimension counting arguments to prove the following:

Proposition A.2. Let the discrete data $\mathcal{D}$ be given. The set of solutions of (A.21) and (A.22) forms a one-dimensional linear subspace of $\Lambda_{N, M}$. The general solution of $(A .21)$ and (A.22) is given by the one-parameter family of matrices (A.18), indexed by the complex parameter c. Thus, $\Lambda_{N, M}^{\mathcal{D}} \simeq \mathbb{C}$, with the isomorphism being established via the complex constant $c$. In particular, if $c$ is given, then the coefficient functions $\boldsymbol{F}^{(p)}(x, t)$ are uniquely determined as functions of $x$ and $t$, and if $c$ is chosen to be zero, then $\boldsymbol{F}(x, t, \lambda)$ is the zero matrix. 
This proposition allows us to index the elements of $\Lambda_{N, M}^{\mathcal{D}}$ by the constant $c$ which is now a genuine coordinate for the one-dimensional subspace $\Lambda_{N, M}^{\mathcal{D}}$. We indicate the dependence by writing $\boldsymbol{F}_{\mathcal{D}, c}(x, t, \lambda)$ for the matrices in this subspace.

This proposition is true even if homogeneous constraints less structured than (A.21) and (A.22) are imposed. In order for the dimension count to come out right it is sufficient to choose $M \cdot(N+1)$ arbitrary complex numbers $\lambda_{k}$ along with corresponding constant vectors $\vec{c}^{(k)} \in \mathbb{C}^{N+1}$ (the numbers $\lambda_{k}$ need not all be distinct, as long as the vectors $\vec{c}$ belonging to each $\lambda_{k}$ are linearly independent) and to impose $\boldsymbol{F}_{\mathcal{D}, c}\left(x, t, \lambda_{k}\right) \vec{c}^{(k)}=\overrightarrow{0}$ for all $k=1, \ldots, M \cdot(N+1)$. The additional structure in the constraints (A.21) and (A.22) is needed for the following.

Proposition A.3. Let discrete data $\mathcal{D}$ be given, and let $\boldsymbol{F}_{\mathcal{D}, c}(x, t, \lambda) \in \Lambda_{N, M}^{\mathcal{D}}$. Then

$$
\frac{1}{c} F_{k+1,1}^{(M-1)}(x, t)=-\left(\frac{1}{c} F_{1, k+1}^{(M-1)}(x, t)\right)^{*} \quad k=1, \ldots, N .
$$

We will have use for this symmetry property below. Its proof is simple.

Proof of proposition A.3. It is sufficient to consider the case of $c=1$, since the coefficient matrices simply scale with $c$. It will be convenient to introduce the block form of the coefficient matrices

$$
\boldsymbol{F}^{(p)}(x, t)=\left[\begin{array}{ll}
a^{(p)} & \vec{b}^{(p) T} \\
\vec{c}^{(p)} & D^{(p)}
\end{array}\right]
$$

where $a^{(p)}(x, t)$ is a scalar, $\vec{b}^{(p)}(x, t)$ and $\vec{c}^{(p)}(x, t)$ are $N$-component vectors, and $\boldsymbol{D}^{(p)}(x, t)$ is an $N \times N$ matrix. We will prove the stronger result that for all $p=0, \ldots, M-1$,

$$
\vec{c}^{(p)}(x, t)=-\vec{b}^{(p)}(x, t)^{*} .
$$

In this form, equations (A.21) and (A.22) take the form of the system

$$
\begin{aligned}
& -\lambda_{k}^{M}+\sum_{p=0}^{M-1} \lambda_{k}^{p}\left(\vec{b}^{(p) T} \vec{g}^{(k) *} \mathrm{e}^{2 \mathrm{i}\left(\lambda_{k} x+\lambda_{k}^{2} t\right)}-a^{(p)}\right)=0 \\
& \lambda_{k}^{M} \vec{g}^{(k) *}+\sum_{p=0}^{M-1} \lambda_{k}^{p}\left(\boldsymbol{D}^{(p)} \vec{g}^{(k) *}-\mathrm{e}^{-2 \mathrm{i}\left(\lambda_{k} x+\lambda_{k}^{2} t\right)} \vec{c}^{(p)}\right)=\overrightarrow{0} \\
& \lambda_{k}^{* M} \vec{g}^{(k)}+\sum_{p=0}^{M-1} \lambda_{k}^{* p}\left(a^{(p)} \vec{g}^{(k)}+\mathrm{e}^{2 \mathrm{i}\left(\lambda_{k}^{*} x+\lambda_{k}^{* 2} t\right)} \vec{b}^{(p)}\right)=\overrightarrow{0} \\
& \lambda_{k}^{* M} \mathbb{I}_{N}+\sum_{p=0}^{M-1} \lambda_{k}^{* p}\left(\mathrm{e}^{-2 \mathrm{i}\left(\lambda_{k}^{*} x+\lambda_{k}^{* 2} t\right)} \vec{c}^{(p)} \vec{g}^{(k) T}+\boldsymbol{D}^{(p)}\right)=\mathbf{0}
\end{aligned}
$$

where $k=1, \ldots, M$. From the first and fourth equations, we can eliminate $a^{(p)}(x, t)$ and $D^{(p)}(x, t), p=0, \ldots, M-1$ in favour of the $\vec{b}^{(p)}(x, t)$ and $\vec{c}^{(p)}(x, t)$. This involves introducing the elements of the inverse $\boldsymbol{W}$ of the Vandermonde matrix $\boldsymbol{V}$ having elements $V_{j k} \doteq \lambda_{j}^{k-1}$, but it leads to two decoupled linear systems, one for the $\vec{b}^{(p)}(x, t)$ and the other for the $\vec{c}^{(p)}(x, t)$. These systems are

$$
\begin{aligned}
& \sum_{r=1}^{M} H_{k r} \vec{c}^{(r-1)}=h_{k} \\
& \sum_{r=1}^{M} H_{k r}^{*} \vec{b}^{(r-1)}=-h_{k}^{*}
\end{aligned}
$$


where

$$
H_{k r} \doteq V_{k r} \mathrm{e}^{-2 \mathrm{i}\left(\lambda_{k} x+\lambda_{k}^{2} t\right)}+\sum_{s=1}^{M} V_{k s} \sum_{j=1}^{M} W_{s j}^{*} V_{j r}^{*} \mathrm{e}^{-2 \mathrm{i}\left(\lambda_{j}^{*} x+\lambda_{j}^{*} t\right)} \vec{g}^{(j) T} \vec{g}^{(k) *}
$$

and

$$
h_{k} \doteq \lambda_{k}^{M} \vec{g}^{(k) *}-\sum_{s=1}^{M} \sum_{j=1}^{M} V_{k s} W_{s j}^{*} \lambda_{j}^{* M} \vec{g}^{(k) *} .
$$

It is then clear that $\vec{c}^{(p)}=-\vec{b}^{(p) *}$ for all $p$.

So, the emphasis has changed with respect to these matrices and their relation to the functions $q_{k}(x, t)$. Rather than determining the coefficient matrices $\boldsymbol{F}^{(p)}(x, t)$ from a given set of functions $q_{k}(x, t)$ solving (A.5) by an asymptotic expansion procedure, we are determining them from the discrete data $\mathcal{D}$ and a choice of the constant $c$. If there is to be any consistency, then we must still have relations between the coefficient matrices $\boldsymbol{F}^{(p)}(x, t)$ of $\boldsymbol{F}_{\mathcal{D}, c}$ and the functions $q_{k}(x, t)$; in particular, we can rewrite (A.7),

$$
q_{k}(x, t) \doteq \frac{2 \mathrm{i}}{c} F_{1, k+1}^{(M-1)}(x, t)
$$

and use it as a definition of some functions $q_{k}(x, t)$ in terms of the discrete data $\mathcal{D}$ and the constant $c$. Note that as long as $c \neq 0$, then this definition is actually independent of $c$ because $\boldsymbol{F}_{\mathcal{D}, c}$ is directly proportional to $c$. The fact that (A.30) is sensible as a definition of the $q_{k}(x, t)$ is shown by

Proposition A.4. Let the discrete data $\mathcal{D}$ be given and let the constant $c$ be non-zero, and let the functions $q_{1}(x, t), \ldots, q_{N}(x, t)$ be defined (in terms of $\mathcal{D}$ alone) via (A.30). This determines the operators $\mathcal{X}(\lambda, \vec{q})$ and $\mathcal{T}(\lambda, \vec{q})$. Then for any $\boldsymbol{F}_{\mathcal{D}, c}(x, t, \lambda) \in \Lambda_{N, M}^{\mathcal{D}}$,

$$
\mathcal{X}(\lambda, \vec{q}) \boldsymbol{F}_{\mathcal{D}, c}(\lambda, x, t)=\mathcal{T}(\lambda, \vec{q}) \boldsymbol{F}_{\mathcal{D}, c}(\lambda, x, t)=\mathbf{0} .
$$

For these $q_{k}(x, t)$ the columns of $\boldsymbol{F}_{\mathcal{D}, c}(x, t, \lambda)$ are generically linearly independent and therefore form a basis of the common nullspace $K$ for almost all $\lambda$.

Recall that the commutator $[\mathcal{X}, \mathcal{T}]$ is, for fixed $x$ and $t$, a matrix multiplication operator in $\mathbb{C}^{N+1}$. Thus, the existence of the common nullspace $K$ of $\mathcal{X}(\lambda, \vec{q})$ and $\mathcal{T}(\lambda, \vec{q})$ of generic dimension $N+1$ for these functions $q_{k}(x, t)$ implies the vanishing of the commutator and the compatibility of the two linear problems. Therefore, we have

Corollary A.1. The functions $q_{k}(x, t)$ constructed from any set of discrete data $\mathcal{D}$ satisfy the vector nonlinear Schrödinger equation (A.5).

A time-dependent self-consistent potential function $V_{0}(x, t)$ generated from the functions $q_{k}(x, t)$ connected with a set of discrete data $\mathcal{D}$ according to (A.8) will be called a separable potential [22].

Proof of proposition A.4. Let $\boldsymbol{F}_{\mathcal{D}, c}(x, t, \lambda) \in \Lambda_{N, M}^{\mathcal{D}}$. The proof begins with the simple observation that, as a consequence of the vectors $\vec{a}^{(k)}$ and $\vec{b}^{(k, j)}$ in the homogeneous relations (A.21) and (A.22) satisfied by $\boldsymbol{F}_{\mathcal{D}, c}(x, t, \lambda)$ being independent of $x$ and $t$, these relations are satisfied by $\left(\mathcal{X} \boldsymbol{F}_{\mathcal{D}, c}\right)(x, t, \lambda)$ and $\left(\mathcal{T} \boldsymbol{F}_{\mathcal{D}, c}\right)(x, t, \lambda)$ as well. For example, with the operator $\mathcal{X}$,

$\left(\mathcal{X}(\lambda, \vec{q}) \boldsymbol{F}_{\mathcal{D}, c}\right)\left(x, t, \lambda_{k}\right) \vec{a}^{(k)}=\mathcal{X}\left(\lambda_{k}, \vec{q}\right)\left(\boldsymbol{F}_{\mathcal{D}, c}\left(x, t, \lambda_{k}\right) \vec{a}^{(k)}\right)=\mathcal{X}\left(\lambda_{k}, \vec{q}\right) \mathbf{0}=\mathbf{0}$ 
for $k=1, \ldots, M$, and

$$
\left(\mathcal{X}(\lambda, \vec{q}) \boldsymbol{F}_{\mathcal{D}, c}\right)\left(x, t, \lambda_{k}^{*}\right) \vec{b}^{(k, j)}=\mathcal{X}\left(\lambda_{k}^{*}, \vec{q}\right)\left(\boldsymbol{F}_{\mathcal{D}, c}\left(x, t, \lambda_{k}^{*}\right) \vec{b}^{(k, j)}\right)=\mathcal{X}\left(\lambda_{k}^{*}, \vec{q}\right) \mathbf{0}=\mathbf{0}
$$

for $k=1, \ldots, M$ and $j=1, \ldots, N$. The argument is unchanged if $\mathcal{X}$ is replaced with $\mathcal{T}$.

Next, we examine the form of the matrix $\left(\mathcal{X}(\lambda, \vec{q}) \boldsymbol{F}_{\mathcal{D}, c}\right)(x, t, \lambda)$. It is straightforward to see that

$$
\begin{aligned}
\left(\mathcal{X}(\lambda, \vec{q}) \boldsymbol{F}_{\mathcal{D}, c}\right)(x, t, \lambda)=\left\{\lambda^{M}\left(2 \mathrm{i}\left[\boldsymbol{E}, \boldsymbol{F}^{(M-1)}\right]-c \boldsymbol{U}\right)\right. \\
+\sum_{p=1}^{M-1} \lambda^{p}\left(\partial_{x} \boldsymbol{F}^{(p)}+2 \mathrm{i}\left[\boldsymbol{E}, \boldsymbol{F}^{(p-1)}\right]-\boldsymbol{U} \boldsymbol{F}^{(p)}\right) \\
\left.+\left(\partial_{x} \boldsymbol{F}^{(0)}-\boldsymbol{U} \boldsymbol{F}^{(0)}\right)\right\} \exp \left(-2 \mathrm{i}\left(\lambda x+\lambda^{2} t\right) \boldsymbol{E}\right) .
\end{aligned}
$$

Now, as a consequence of the definition of the functions $q_{k}(x, t)$ in terms of the discrete data $\mathcal{D}$ and the symmetry property guaranteed by proposition A.3, the leading term vanishes identically, that is,

$$
2 \mathrm{i}\left[\boldsymbol{E}, \boldsymbol{F}^{(M-1)}(x, t)\right]=c \boldsymbol{U}(\vec{q}) .
$$

This, along with the fact that $\mathcal{X}(\lambda, \vec{q}) \boldsymbol{F}_{\mathcal{D}, c}(x, t, \lambda)$ satisfies the homogeneous conditions (A.21) and (A.22), means that

$$
\mathcal{X}(\lambda, \vec{q}) \boldsymbol{F}_{\mathcal{D}, c}(x, t, \lambda) \in \Lambda_{N, M}^{\mathcal{D}} .
$$

Not only that, but for matrices in $\Lambda_{N, M}^{\mathcal{D}}$ the only way that the coefficient of $\lambda^{M}$ can vanish is for the leading constant to vanish. Therefore, by the isomorphism between $\Lambda_{N, M}^{\mathcal{D}}$ and $\mathbb{C}$ via the leading constant, it follows that

$$
\mathcal{X}(\lambda, \vec{q}) \boldsymbol{F}_{\mathcal{D}, c}(x, t, \lambda)=\mathbf{0} .
$$

We now consider the form of $\mathcal{T}(\lambda, \vec{q}) \boldsymbol{F}_{\mathcal{D}, c}(x, t, \lambda)$,

$$
\begin{aligned}
\mathcal{T}(\lambda, \vec{q}) \boldsymbol{F}_{\mathcal{D}, c}( & x, t, \lambda)=\left\{\lambda^{M+1}\left(2 \mathrm{i}\left[\boldsymbol{E}, \boldsymbol{F}^{(M-1)}\right]-c \boldsymbol{U}\right)\right. \\
& +\lambda^{M}\left(2 \mathrm{i}\left[\boldsymbol{E}, \boldsymbol{F}^{(M-2)}\right]-\boldsymbol{U} \boldsymbol{F}^{(M-1)}-\frac{1}{2} \mathrm{i} c \boldsymbol{V}\right) \\
& +\sum_{p=2}^{M-1} \lambda^{p}\left(\partial_{t} \boldsymbol{F}^{(p)}+2 \mathrm{i}\left[\boldsymbol{E}, \boldsymbol{F}^{(p-2)}\right]-\boldsymbol{U} \boldsymbol{F}^{(p-1)}-\frac{1}{2} \mathrm{i} \boldsymbol{V} \boldsymbol{F}^{(p)}\right) \\
& +\lambda\left(\partial_{t} \boldsymbol{F}^{(1)}-\boldsymbol{U} \boldsymbol{F}^{(0)}-\frac{1}{2} \mathrm{i} \boldsymbol{V} \boldsymbol{F}^{(1)}\right) \\
& \left.+\left(\partial_{t} \boldsymbol{F}^{(0)}-\frac{1}{2} \mathrm{i} \boldsymbol{V} \boldsymbol{F}^{(0)}\right)\right\} \exp \left(-2 \mathrm{i}\left(\lambda x+\lambda^{2} t\right) \boldsymbol{E}\right) .
\end{aligned}
$$

Once again, the definition of the functions $q_{k}(x, t)$ and the symmetry property of proposition A.3 guarantee that the coefficient of $\lambda^{M+1}$ vanishes. We shall now show that the coefficient of $\lambda^{M}$ vanishes as well. Begin by writing $\boldsymbol{F}^{(M-1)}(x, t)$ in the block form

$$
\boldsymbol{F}^{(M-1)}(x, t)=\left[\begin{array}{ll}
a^{(M-1)} & \vec{b}^{(M-1) T} \\
\vec{c}^{(M-1)} & D^{(M-1)}
\end{array}\right] .
$$


We already know by definition of the functions $q_{k}(x, t)$ and proposition A.3, that $\vec{b}^{(M-1) T}=$ $\vec{q}^{T} / 2 \mathrm{i}$ and $\vec{c}^{(M-1)}=-\vec{b}^{(M-1) *}$. Making use of the fact that all of the terms in (A.34) vanish identically, we also have

$$
\partial_{x} \boldsymbol{F}^{(M-1)}(x, t)+2 \mathrm{i}\left[\boldsymbol{E}, \boldsymbol{F}^{(M-2)}(x, t)\right]-\boldsymbol{U}(\vec{q}) \boldsymbol{F}^{(M-1)}(x, t)=\mathbf{0} .
$$

This implies that for the coefficient of $\lambda^{M}$ in (A.38) to vanish, it will be enough to show that

$$
c \boldsymbol{V}(\vec{q})=2 \mathrm{i} \partial_{x} \boldsymbol{F}^{(M-1)}(x, t) .
$$

From (A.4), it is clear (A.41) is satisfied in the off-diagonal blocks. To show that the diagonal blocks also vanish, we write out the diagonal blocks of (A.40)

$$
\left[\begin{array}{cc}
\partial_{x} a^{(M-1)} & \overrightarrow{0}^{T} \\
\overrightarrow{0} & \partial_{x} \boldsymbol{D}^{(M-1)}
\end{array}\right]-\left[\begin{array}{cc}
\vec{q}^{T} \vec{c}^{(M-1)} & \overrightarrow{0}^{T} \\
\overrightarrow{0} & -\vec{q}^{*} \vec{b}^{(M-1) T}
\end{array}\right]=\mathbf{0} .
$$

Eliminating the derivatives of $a^{(M-1)}$ and $D^{(M-1)}$ between this equation and the diagonal blocks of (A.41) and comparing with the definition (A.4) of $\boldsymbol{V}(\vec{q})$, we finally see that (A.41) is satisfied identically. By similar arguments as we used above, it follows that $\mathcal{T}(\lambda, \vec{q}) \boldsymbol{F}_{\mathcal{D}, c}(x, t, \lambda)$ is also the zero element of $\Lambda_{N, M}^{\mathcal{D}}$. This ends the proof of the proposition.

We now return to the problem of interest, namely the algebraic construction of separable time-dependent potentials for the linear Schrödinger equation and of a large number of exact solutions to this linear equation. From the construction of the subspace $\Lambda_{N, M}^{\mathcal{D}}$ we can extract a simpler construction of the quantities of immediate interest, and cast the whole procedure in the form of an algorithm. The key observation is that it is sufficient to build from given discrete data $\mathcal{D}$ only the first row of a matrix $\boldsymbol{F}_{\mathcal{D}, c}(x, t, \lambda)$ in the space $\Lambda_{N, M}^{\mathcal{D}}$. This gives us both the functions $q_{k}(x, t)$ via the first row of the coefficient matrix $\boldsymbol{F}^{(M-1)}(x, t)$ from which we find the potential $V_{0}(x, t)$ and also the image of the projection operator $\mathcal{P}$ that consists of solutions of the linear Schrödinger equation with this potential.

So we consider the first row of $\boldsymbol{F}_{\mathcal{D}, c}(x, t, \lambda)$ and impose the homogeneous linear constraints (A.21) and (A.22). Introducing

$$
a(x, t, \lambda)=F_{11}(x, t, \lambda)=\left(\lambda^{M}+\sum_{p=0}^{M-1} \lambda^{p} a^{(p)}(x, t)\right) \mathrm{e}^{-2 \mathrm{i}\left(\lambda x+\lambda^{2} t\right)}
$$

and

$$
\vec{b}(x, t, \lambda)=\left(F_{12}(x, t, \lambda), \ldots, F_{1, N+1}(x, t, \lambda)\right)^{T}=\sum_{p=0}^{M-1} \lambda^{p} \vec{b}^{(p)}(x, t)
$$

the relations (A.21) and (A.22) take the simple form

$$
\begin{aligned}
& a\left(x, t, \lambda_{k}\right)=\vec{g}^{(k) \dagger} \vec{b}\left(x, t, \lambda_{k}\right) \\
& \vec{b}\left(x, t, \lambda_{k}^{*}\right)=-a\left(x, t, \lambda_{k}^{*}\right) \vec{g}^{(k)}
\end{aligned}
$$

where $k=1, \ldots, M$. Note that without loss of generality, we are taking $c=1$. Written out in its entirety, this is a square linear system for the $M \cdot(N+1)$ unknowns, $a^{(p)}(x, t)$, and the $N$ elements of $\vec{b}^{(p)}(x, t)$ for $p=0, \ldots, M-1$. The matrix of this system, and the right-hand side, are explicit functions of $x$ and $t$ through the exponential functions contributed by $a\left(x, t, \lambda_{k}\right)$ and $a\left(x, t, \lambda_{k}^{*}\right)$. 
From the solution of this linear system, one computes the potential function as

$$
V_{0}(x, t)=-4 \sum_{n=1}^{N}\left|b_{n}^{(M-1)}(x, t)\right|^{2} .
$$

Then, we see that $a(x, t, \lambda)$ and all the elements of $\vec{b}(x, t, \lambda)$ are solutions of the linear equation

$$
\mathrm{i} \partial_{t} f+\frac{1}{2} \partial_{x}^{2} f-V_{0}(x, t) f=0
$$

for fixed but arbitrary $\lambda$. Being polynomial in $\lambda$, each element of $\vec{b}(x, t, \lambda)$ sweeps out an $M$-dimensional space of solutions as $\lambda$ varies. The solutions contained in $a(x, t, \lambda)$ are more interesting because the presence of the exponential means that all of these solutions for real $\lambda$ are linearly independent. This immediately gives an infinite-dimensional space of solutions to the linear Schrödinger equation.

In fact, the function $a(x, t, \lambda)$ contains an $L^{2}(\mathbb{R})$ basis of solutions of the Schrödinger equation as the parameter $\lambda$ is varied [22]. In particular, the set

$$
\left\{a\left(x, t, \lambda_{1}^{*}\right), \ldots, a\left(x, t, \lambda_{M}^{*}\right), a(x, t, \lambda), \lambda \text { real }\right\}
$$

considered as functions of $x$ for fixed $t$, is complete. For real $\lambda$, set

$$
\Psi_{\mathrm{d}}(x, t, \lambda) \doteq\left(\pi \prod_{k=1}^{M}\left|\lambda-\lambda_{k}\right|^{2}\right)^{-1 / 2} a(x, t, \lambda) .
$$

The subscript ' $d$ ' indicates solutions that superpose to form dispersive waves. For $\lambda$ and $\eta$ real we then have the inner products

$$
\left\langle\Psi_{\mathrm{d}}(\cdot, t, \lambda), \Psi_{\mathrm{d}}(\cdot, t, \eta)\right\rangle=\delta(\lambda-\eta)
$$

and for $k=1, \ldots, M$,

$$
\left\langle a\left(\cdot, t, \lambda_{k}^{*}\right), \Psi_{\mathrm{d}}(\cdot, t, \mu)\right\rangle=0 .
$$

Also, $\operatorname{dim} \operatorname{span}\left\{a\left(x, t, \lambda_{k}^{*}\right), k=1, \ldots, M\right\}=M$ as functions of $x$ for fixed $t$. So, let $\left\{\Psi_{\mathrm{b}, k}(x, t)\right\}$ be any basis of $\operatorname{span}\left\{a\left(x, t, \lambda_{k}^{*}\right), k=1, \ldots, M\right\}$ that is orthonormal with respect to the inner product (say obtained by the Gram-Schmidt procedure), so that

$$
\left\langle\Psi_{\mathrm{b}, j}(\cdot, t), \Psi_{\mathrm{b}, k}(\cdot, t)\right\rangle=\delta_{j k} .
$$

The subscript ' $b$ ' indicates solutions that are bound and have finite energy. Note that this basis remains orthonormal because the time evolution of these functions under (A.47) is unitary.

The completeness relation is generalized to $L^{2}(\mathbb{R})$ from that proved in [22] as

Proposition A.5. Let discrete data $\mathcal{D}$ be given and let $t \in \mathbb{R}$ be fixed. For all $f(x) \in L^{2}(\mathbb{R})$, we have the expansion

$$
f(x)=\int_{-\infty}^{\infty} f_{\mathrm{d}}(\lambda, t) \Psi_{\mathrm{d}}(x, t, \lambda) \mathrm{d} \lambda+\sum_{k=1}^{M} f_{\mathrm{b}, k}(t) \Psi_{\mathrm{b}, k}(x, t)
$$

where the expansion coefficients are given by

$$
f_{\mathrm{d}}(\lambda, t)=\left\langle\Psi_{\mathrm{d}}(\cdot, t, \lambda), f(\cdot)\right\rangle \quad f_{\mathrm{b}, k}(t)=\left\langle\Psi_{\mathrm{b}, k}(\cdot, t), f(\cdot)\right\rangle .
$$

The orthogonality relations for the functions $\Psi_{\mathrm{b}, k}(x, t)$ and $\Psi_{\mathrm{d}}(x, t, \lambda)$ are implied by this result. Note that if $f=f(x, t)$ satisfies (A.47) then the expansion coefficients are independent of $t$ and can be constructed from the initial data $f(x, 0)$. Thus one solves the initial-value problem for (A.47) in $L^{2}(\mathbb{R})$. 


\section{Appendix B. Dispersive local decay estimates}

Here, we establish several important properties of the unitary group $\mathrm{e}^{-\mathrm{i} t \mathcal{B}}$. We will consider even perturbations of the even two-soliton periodic potentials, so we will work in either the even or odd subspace of $L^{2}(\mathbb{R})$. For a given function $f(\cdot)$ in $L^{2}(\mathbb{R})$, the operator $\mathcal{P}_{\mathrm{c}}^{(\mathrm{e}, \mathrm{o})}$ is defined as the spectral projection onto the continuous part of the spectrum of $\mathcal{B}$

$$
\left(\mathcal{P}_{\mathrm{c}}^{(\mathrm{e}, \mathrm{o})} f\right)(x) \doteq \int_{0}^{\infty}\left\langle\Psi_{\mathrm{d}}^{(\mathrm{e}, \mathrm{o})}(\cdot, 0, \lambda), f(\cdot)\right\rangle \Psi_{\mathrm{d}}^{(\mathrm{e}, \mathrm{o})}(x, 0, \lambda) \mathrm{d} \lambda .
$$

As we will now see, the main difference between the even and odd cases is in the rate of dispersive decay, and the difference can be directly traced to the behaviour of the dispersive eigenfunction $\Psi_{\mathrm{d}}^{(\mathrm{e}, \mathrm{o})}(x, 0, \lambda)$ in the vicinity of $\lambda=0$. It is easy to see from the explicit formulae that the eigenfunctions are continuous in $\lambda$ at $\lambda=0$, and that the odd mode vanishes there

$$
\Psi_{\mathrm{d}}^{(\mathrm{o})}(x, 0, \lambda=0)=0
$$

while the even mode does not vanish, but is simply finite at $\lambda=0$. We say that the existence of a non-trivial eigenfunction at $\lambda=0$, as in the even case, indicates a zero-energy resonance of the system. The ubiquitous effect of a zero-energy resonance is to alter the rate of dispersive decay in the system. However, more dramatic effects can appear if under the influence of a perturbation, the zero-energy resonance is directly excited. This latter situation we refer to as a parametric zero-energy resonance. A system with a zero-energy resonance is 'primed' to feel the effects of a parametric zero-energy resonance in the presence of an appropriate perturbation.

\section{B.1. Non-singular local decay}

First, we will prove the non-singular local decay estimate for the unitary group $\mathrm{e}^{-\mathrm{i} t \mathcal{B}}$.

Proposition B.1. Fix $\sigma>\frac{5}{2}$. There exist constants $L^{(\mathrm{e}, \mathrm{o})}>0$ such that

$$
\left\|\langle\cdot\rangle^{-\sigma}\left(\mathrm{e}^{-\mathrm{i} t \mathcal{B}} \mathcal{P}_{\mathrm{c}}^{(\mathrm{e})} f\right)(\cdot)\right\|_{2} \leqslant L^{(\mathrm{e})}\langle t\rangle^{-1 / 2}\left\|\langle\cdot\rangle^{\sigma} f(\cdot)\right\|_{2}
$$

and

$$
\left\|\langle\cdot\rangle^{-\sigma}\left(\mathrm{e}^{-\mathrm{i} t \mathcal{B}} \mathcal{P}_{\mathrm{c}}^{(\mathrm{o})} f\right)(\cdot)\right\|_{2} \leqslant L^{(\mathrm{o})}\langle t\rangle^{-3 / 2}\left\|\langle\cdot\rangle^{\sigma} f(\cdot)\right\|_{2}
$$

for all $f \in L^{2}(\mathbb{R})$ for which the right-hand side makes sense.

The proof is based on a sequence of intermediate results. First, from the simple chain of estimates

$$
\left\|\langle\cdot\rangle^{-\sigma} \mathrm{e}^{-\mathrm{i} t \mathcal{B}} \mathcal{P}_{\mathrm{c}}^{(\mathrm{e}, \mathrm{o})} f(\cdot)\right\|_{2} \leqslant\left\|\mathrm{e}^{-\mathrm{i} t \mathcal{B}} \mathcal{P}_{\mathrm{c}}^{(\mathrm{e}, \mathrm{o})} f(\cdot)\right\|_{2}=\left\|\mathcal{P}_{\mathrm{c}}^{(\mathrm{e}, \mathrm{o})} f(\cdot)\right\|_{2} \leqslant\|f(\cdot)\|_{2} \leqslant\left\|\langle\cdot\rangle^{\sigma} f(\cdot)\right\|_{2}
$$

we have

Lemma B.1. For all $\sigma>0$, we have the simple estimate

$$
\left\|\langle\cdot\rangle^{-\sigma} \mathrm{e}^{-\mathrm{i} t \mathcal{B}} \mathcal{P}_{\mathrm{c}}^{(\mathrm{e}, \mathrm{o})} f(\cdot)\right\|_{2} \leqslant\left\|\langle\cdot\rangle^{\sigma} f(\cdot)\right\|_{2}
$$

for all $f \in L^{2}(\mathbb{R})$ for which the right-hand side makes sense. 
We now want to refine the above uniform estimate to include a multiplicative factor of $\left\|\langle\cdot\rangle^{\sigma} f(\cdot)\right\|_{2}$ that decays in $|t|$. To this end, we fix $t \neq 0$ and observe that by the definition of the operator $\mathcal{B}$,

$$
\langle x\rangle^{-\sigma}\left(\mathrm{e}^{-\mathrm{i} t \mathcal{B}} \mathcal{P}_{\mathrm{c}}^{(\mathrm{e}, \mathrm{o})} f\right)(x)=\int_{-\infty}^{\infty}\langle y\rangle^{\sigma} f(y) h(x, y ; t) \mathrm{d} y
$$

where

$$
h(x, y ; t) \doteq\langle x\rangle^{-\sigma}\langle y\rangle^{-\sigma} \int_{0}^{\infty} \Psi_{\mathrm{d}}^{(\mathrm{e}, \mathrm{o})}(y, 0, \lambda)^{*} \Psi_{\mathrm{d}}^{(\mathrm{e}, \mathrm{o})}(x, 0, \lambda) \mathrm{e}^{-2 \mathrm{i} \lambda^{2} t} \mathrm{~d} \lambda .
$$

We note here that the integral in the definition of $h(x, y ; t)$ is improper; the integrand is not absolutely integrable, and the integral from zero to infinity should be interpreted as the limit of the integral from zero to $R$ as $R \uparrow \infty$. This limit exists as long as $t \neq 0$, and consequently the function $h(x, y ; t)$ is well defined for $t \neq 0$. The trouble with the function $h(x, y ; t)$ at $t=0$ is not our concern here because we already have a uniform estimate that holds for all $t$, and, in particular, for $t$ near zero. Thus we will be thinking of $t$ as being large in what follows.

In any case, by Cauchy-Schwarz, we have

$$
\left|\langle x\rangle^{-\sigma}\left(\mathrm{e}^{-\mathrm{i} t \mathcal{B}} \mathcal{P}_{\mathrm{c}}^{(\mathrm{e}, \mathrm{o})} f\right)(x)\right| \leqslant\|h(x, \cdot ; t)\|_{2}\left\|\langle\cdot\rangle^{\sigma} f(\cdot)\right\|_{2} .
$$

It follows that

$$
\left\|\langle\cdot\rangle^{-\sigma}\left(\mathrm{e}^{-\mathrm{i} t \mathcal{B}} \mathcal{P}_{\mathrm{c}}^{(\mathrm{e}, \mathrm{o})} f\right)(\cdot)\right\|_{2} \leqslant\|h(\cdot, \cdot ; t)\|_{2}\left\|\langle\cdot\rangle^{\sigma} f(\cdot)\right\|_{2}
$$

an estimate that involves the Hilbert-Schmidt norm of the kernel $h(x, y ; t)$ for each fixed $t$. The rest of our work will be to show $h(x, y ; t)$ is in $L^{2}\left(\mathbb{R}^{2}\right)$ for each fixed $t$, with its norm decaying in $|t|$.

First note that from the explicit formulae

$$
\begin{aligned}
& \Psi_{\mathrm{d}}^{(\mathrm{o})}(x, 0, \lambda)=\frac{2 \lambda a^{(1)}(x, 0) \cos (2 \lambda x)-2 \mathrm{i}\left(\lambda^{2}+a^{(0)}(x, 0)\right) \sin (2 \lambda x)}{\sqrt{2 \pi\left(\lambda^{2}+\rho_{1}^{2}\right)\left(\lambda^{2}+\rho_{2}^{2}\right)}} \\
& \Psi_{\mathrm{d}}^{(\mathrm{e})}(x, 0, \lambda)=\frac{2\left(\lambda^{2}+a^{(0)}(x, 0)\right) \cos (2 \lambda x)-2 \mathrm{i} \lambda a^{(1)}(x, 0) \sin (2 \lambda x)}{\sqrt{2 \pi\left(\lambda^{2}+\rho_{1}^{2}\right)\left(\lambda^{2}+\rho_{2}^{2}\right)}}
\end{aligned}
$$

where $a^{(0)}(x, t)$ and $a^{(1)}(x, t)$ are bounded analytic functions of $x$, we obtain

Lemma B.2. Let the parameters $\rho_{1}$ and $\rho_{2}$ be fixed. The function defined by

$$
q(\lambda) \doteq \Psi_{\mathrm{d}}^{(\mathrm{e}, \mathrm{o})}(x, 0, \lambda) \Psi_{\mathrm{d}}^{(\mathrm{e}, \mathrm{o})}(y, 0, \lambda)^{*}
$$

is in $C^{k}\left(\mathbb{R}_{+}\right)$for all $k \geqslant 0$. In particular, all derivatives with respect to $\lambda$ are uniformly bounded functions of $\lambda$. The norms $\left\|q^{(k)}(\cdot)\right\|_{\infty}$ are homogeneous polynomials in $|x|$ and $|y|$ of degree $k$, with non-negative coefficients that depend only on $\rho_{1}$ and $\rho_{2}$. Also, in the odd case, we have $q(\lambda)=\mathrm{O}\left(\lambda^{2}\right)$ for $\lambda$ near zero, while in the even case $q(\lambda)=\mathrm{O}(1)$.

In showing that $h(x, y ; t)$ is $L^{2}\left(\mathbb{R}^{2}\right)$ with its norm decaying in $t$, we will find that the main contribution for large $t$ comes from the part of the integral near $\lambda=0$. To see this, we first separate the contributions near and away from zero. Let $g_{\Delta}(\lambda)$ be a non-negative 'bump function', infinitely differentiable for real $\lambda>0$, identically equal to 1 for $0 \leqslant \lambda \leqslant \Delta / 2$ and identically equal to zero for $\lambda \geqslant 3 \Delta / 2$. Let $\tilde{g}_{\Delta}(\lambda) \doteq 1-g_{\Delta}(\lambda)$. Then

$$
h(x, y ; t)=h_{\Delta}(x, y ; t)+\tilde{h}_{\Delta}(x, y ; t)
$$


where

$h_{\Delta}(x, y ; t) \doteq\langle x\rangle^{-\sigma}\langle y\rangle^{-\sigma} \int_{0}^{3 \Delta / 2} \Psi_{\mathrm{d}}^{(\mathrm{e}, \mathrm{o})}(y, 0, \lambda)^{*} \Psi_{\mathrm{d}}^{(\mathrm{e}, \mathrm{o})}(x, 0, \lambda) g_{\Delta}(\lambda) \mathrm{e}^{-2 \mathrm{i} \lambda^{2} t} \mathrm{~d} \lambda$

and

$\tilde{h}_{\Delta}(x, y ; t) \doteq\langle x\rangle^{-\sigma}\langle y\rangle^{-\sigma} \int_{\Delta / 2}^{\infty} \Psi_{\mathrm{d}}^{(\mathrm{e}, \mathrm{o})}(y, 0, \lambda)^{*} \Psi_{\mathrm{d}}^{(\mathrm{e}, \mathrm{o})}(x, 0, \lambda) \tilde{g}_{\Delta}(\lambda) \mathrm{e}^{-2 \mathrm{i} \lambda^{2} t} \mathrm{~d} \lambda$.

First, we will show that away from $\lambda=0$, we can obtain an arbitrary decay in time.

Lemma B.3. Fix $L>0$. For some $k \geqslant 2$, suppose that $f(\lambda)$ is in $C^{n}([L, \infty])$ for all $n=0,1, \ldots, k$. Suppose that $f(L)=f^{\prime}(L)=\cdots=f^{(k-1)}(L)=0$ and that the limit

$$
\lim _{R \uparrow \infty} \int_{L}^{R} f(\lambda) \mathrm{e}^{-2 \mathrm{i} \lambda^{2} t} \mathrm{~d} \lambda
$$

exists for $t \neq 0$. Then

$$
\left|\lim _{R \uparrow \infty} \int_{L}^{R} f(\lambda) \mathrm{e}^{-2 i \lambda^{2} t} \mathrm{~d} \lambda\right| \leqslant \frac{1}{L 4^{k}|t|^{k}} \sup _{\lambda>L}\left|\lambda^{2}\left(\mathcal{A}^{k} f\right)(\lambda)\right|
$$

where the operator $\mathcal{A}$ is defined by

$$
(\mathcal{A} f)(\lambda) \doteq \frac{\partial}{\partial \lambda}\left(\frac{f(\lambda)}{\lambda}\right) .
$$

Proof. Integrating by parts $k$ times,

$$
\begin{gathered}
\lim _{R \uparrow \infty} \int_{L}^{R} f(\lambda) \mathrm{e}^{-2 \mathrm{i} \lambda^{2} t} \mathrm{~d} \lambda=\lim _{R \uparrow \infty}\left[\left.\sum_{n=0}^{k-1}\left(\frac{\mathrm{i}}{4 t}\right)^{n+1}(-1)^{n} \lambda^{-1} \mathrm{e}^{-2 \mathrm{i} \lambda^{2} t}\left(\mathcal{A}^{n} f\right)(\lambda)\right|_{\lambda=L} ^{\lambda=R}\right. \\
\left.+\left(\frac{-\mathrm{i}}{4 t}\right)^{k} \int_{L}^{R}\left(\mathcal{A}^{k} f\right)(\lambda) \mathrm{e}^{-2 \mathrm{i} \lambda^{2} t} \mathrm{~d} \lambda\right] .
\end{gathered}
$$

The boundary terms at $\lambda=L$ vanish identically, and those at $\lambda=R$ tend to zero as $R \uparrow \infty$. These facts prove the existence of the limit of the integral in the second line, and we find

$$
\begin{gathered}
\left|\lim _{R \uparrow \infty} \int_{L}^{R} f(\lambda) \mathrm{e}^{-2 \mathrm{i} \lambda^{2} t} \mathrm{~d} \lambda\right|=\frac{1}{4^{k}|t|^{k}}\left|\lim _{R \uparrow \infty} \int_{L}^{R}\left(\mathcal{A}^{k} f\right)(\lambda) \mathrm{e}^{-2 \mathrm{i} \lambda^{2} t} \mathrm{~d} \lambda\right| \\
\leqslant \frac{1}{4^{k}|t|^{k}} \lim _{R \uparrow \infty} \int_{L}^{R}\left|\lambda^{2}\left(\mathcal{A}^{k} f\right)(\lambda)\right| \frac{\mathrm{d} \lambda}{\lambda^{2}} \\
\leqslant \frac{1}{L 4^{k}|t|^{k}} \sup _{\lambda>L}\left|\lambda^{2}\left(\mathcal{A}^{k} f\right)(\lambda)\right| .
\end{gathered}
$$

The bound is finite for $k \geqslant 2$.

We can now apply this result to estimate $\tilde{h}_{\Delta}(x, y ; t)$.

Lemma B.4. Fix an integer $k \geqslant 2$, and let $\sigma>k+\frac{1}{2}$. Then, the function $\tilde{h}_{\Delta}(x, y ; t)$ is in $L^{2}\left(\mathbb{R}^{2}\right)$ as a function of $x$ and $y$, with its norm decaying as $|t|^{-k}$. 
Proof. We apply the above lemma with $L=\Delta / 2$ and $f(\lambda)=\Psi_{\mathrm{d}}^{(\mathrm{e}, \mathrm{o})}(x, 0, \lambda) \Psi_{\mathrm{d}}^{(\mathrm{e}, \mathrm{o})}(y, 0, \lambda)^{*}$ $\tilde{g}_{\Delta}(\lambda)$. This gives the pointwise estimate

$\left|\tilde{h}_{\Delta}(x, y ; t)\right| \leqslant \frac{2\langle x\rangle^{-\sigma}\langle y\rangle^{-\sigma}}{\Delta 4^{k}|t|^{k}} \sup _{\lambda>\Delta / 2}\left|\lambda^{2}\left(\mathcal{A}^{k} \Psi_{\mathrm{d}}^{(\mathrm{e}, \mathrm{o})}(x, 0, \cdot) \Psi_{\mathrm{d}}^{(\mathrm{e}, \mathrm{o})}(y, 0, \cdot)^{*} \tilde{g}_{\Delta}(\cdot)\right)(\lambda)\right|$.

The operator $\mathcal{A}^{k}$ acting on the right-hand side makes the supremum bound a polynomial in $|x|$ and $|y|$ of degree $k$. Therefore, for $\tilde{h}_{\Delta}(x, y ; t)$ to lie in $L^{2}\left(\mathbb{R}^{2}\right)$ as a function of $x$ and $y$, it is sufficient to take $\sigma>k+\frac{1}{2}$. The claimed time decay of the $L^{2}$ norm is then obvious. Note that each derivative of $\tilde{g}_{\Delta}(\lambda)$ contributes a factor of order $\mathrm{O}\left(\Delta^{-1}\right)$, so the overall bound on the $L^{2}$ norm of $\tilde{h}_{\Delta}(x, y ; t)$ scales like $\Delta^{-(k+1)}$.

Now, we move on to consider the part of $h(x, y ; t)$ contributed by the neighbourhood of $\lambda=0$. We again need some technical lemmas.

Lemma B.5. For all $\mu \in \mathbb{R}$,

$$
\left|\int_{0}^{\mu} \mathrm{e}^{-2 i \zeta^{2}} \mathrm{~d} \zeta\right| \leqslant \sqrt{3}
$$

Proof. First, note that

$$
\left|\int_{0}^{\mu} \mathrm{e}^{-2 \mathrm{i} \zeta^{2}} \mathrm{~d} \zeta\right| \leqslant \int_{0}^{\mu}|\mathrm{d} \zeta|=|\mu| .
$$

This estimate is useful for bounded $\mu$. Suppose $\mu>M>0$. Then,

$$
\left|\int_{0}^{\mu} \mathrm{e}^{-2 \mathrm{i} \zeta^{2}} \mathrm{~d} \zeta\right| \leqslant M+\left|\int_{M}^{\mu} \mathrm{e}^{-2 \mathrm{i} \zeta^{2}} \mathrm{~d} \zeta\right|
$$

Changing variables to $\tau=\zeta^{2}$ and integrating by parts, one finds

$$
\left|\int_{M}^{\mu} \mathrm{e}^{-2 \mathrm{i} \zeta^{2}} \mathrm{~d} \zeta\right|=\left|\frac{\mathrm{ie}^{-2 i \mu^{2}}}{4 \mu}-\frac{\mathrm{ie}^{-2 i M^{2}}}{4 M}+\frac{\mathrm{i}}{8} \int_{M^{2}}^{\mu^{2}} \mathrm{e}^{-2 \mathrm{i} \tau} \tau^{-3 / 2} \mathrm{~d} \tau\right| \leqslant \frac{3}{4 M} .
$$

Therefore, for $\mu>M>0$, we have the estimate

$$
\left|\int_{0}^{\mu} \mathrm{e}^{-2 \mathrm{i} \zeta^{2}} \mathrm{~d} \zeta\right| \leqslant M+\frac{3}{4 M} .
$$

The right-hand side takes its smallest value, $\sqrt{3}$, for $M_{\min }=\sqrt{3} / 2$. Since for $0<\mu \leqslant M_{\min }$, we have

$$
\left|\int_{0}^{\mu} \mathrm{e}^{-2 \mathrm{i} \zeta^{2}} \mathrm{~d} \zeta\right| \leqslant|\mu| \leqslant M_{\min } \leqslant 2 M_{\min }=\sqrt{3}
$$

the lemma is established uniformly for all positive $\mu$. By symmetry, the same estimate holds for $\mu<0$.

Lemma B.6. Fix $L>0$ and suppose $f(\lambda)$ is twice continuously differentiable, with $f(0)=$ $f^{\prime}(0)=0$ and $f(L)=f^{\prime}(L)=0$. Then

$$
\left|\int_{0}^{L} f(\lambda) \mathrm{e}^{-2 \mathrm{i} \lambda^{2} t} \mathrm{~d} \lambda\right| \leqslant \frac{L \sqrt{3}}{4|t|^{3 / 2}} \sup _{0<\lambda<L}\left|\frac{\partial^{2}}{\partial \lambda^{2}}\left(\frac{f(\lambda)}{\lambda}\right)\right| .
$$


Proof. Integrating by parts using the boundary conditions (evaluations at the lower boundary of $\lambda=0$ are interpreted in the sense of the limit $\lambda \downarrow 0$, that is, from above), we have

$$
\int_{0}^{L} f(\lambda) \mathrm{e}^{-2 \mathrm{i} \lambda^{2} t} \mathrm{~d} \lambda=\frac{\mathrm{i}}{4 t} \int_{0}^{L} \frac{f(\lambda)}{\lambda} \frac{\partial}{\partial \lambda}\left(\mathrm{e}^{-2 \mathrm{i} \lambda^{2} t}\right) \mathrm{d} \lambda=-\frac{\mathrm{i}}{4 t} \int_{0}^{L} \frac{\partial}{\partial \lambda}\left(\frac{f(\lambda)}{\lambda}\right) \mathrm{e}^{-2 \mathrm{i} \lambda^{2} t} \mathrm{~d} \lambda .
$$

Write

$$
\mathrm{e}^{-2 \mathrm{i} \lambda^{2} t}=\frac{\partial}{\partial \lambda} \int_{0}^{\lambda} \mathrm{e}^{-2 \mathrm{i} \sigma^{2} t} \mathrm{~d} \sigma
$$

and integrate by parts again making use of the boundary conditions (with the same caveat as above), to find

$$
\int_{0}^{L} f(\lambda) \mathrm{e}^{-2 \mathrm{i} \lambda^{2} t} \mathrm{~d} \lambda=\frac{\mathrm{i}}{4 t} \int_{0}^{L} \frac{\partial^{2}}{\partial \lambda^{2}}\left(\frac{f(\lambda)}{\lambda}\right) \int_{0}^{\lambda} \mathrm{e}^{-2 \mathrm{i} \sigma^{2} t} \mathrm{~d} \sigma \mathrm{d} \lambda .
$$

With a change of variables to $\zeta=|t|^{1 / 2} \sigma$, this becomes

$$
\int_{0}^{L} f(\lambda) \mathrm{e}^{-2 \mathrm{i} \lambda^{2} t} \mathrm{~d} \lambda=\frac{\mathrm{i}}{4 t|t|^{1 / 2}} \int_{0}^{L} \frac{\partial^{2}}{\partial \lambda^{2}}\left(\frac{f(\lambda)}{\lambda}\right) \int_{0}^{|t|^{1 / 2} \lambda} \mathrm{e}^{-2 \mathrm{i} \zeta^{2}} \mathrm{~d} \zeta \mathrm{d} \lambda .
$$

Estimating the $\lambda$ integral in the obvious way using the uniform bound of the $\zeta$ integral by $\sqrt{3}$ establishes the claimed estimate.

Without the vanishing boundary conditions at $\lambda=0$, one finds less decay in time.

Lemma B.7. Let $f(\lambda)$ be absolutely continuous $0 \leqslant \lambda \leqslant L$, so that $f^{\prime}(\lambda) \in L^{1}([0, L])$. Then

$$
\left|\int_{0}^{L} f(\lambda) \mathrm{e}^{-2 i \lambda^{2} t} \mathrm{~d} \lambda\right| \leqslant\left(|f(0)|+2 \int_{0}^{L}\left|f^{\prime}(\lambda)\right| \mathrm{d} \lambda\right) \frac{\sqrt{3}}{|t|^{1 / 2}}
$$

an order $\mathrm{O}\left(|t|^{-1 / 2}\right)$ bound.

Proof. Separate off the slow decay by writing

$$
\int_{0}^{L} f(\lambda) \mathrm{e}^{-2 \mathrm{i} \lambda^{2} t} \mathrm{~d} \lambda=f(0) \int_{0}^{L} \mathrm{e}^{-2 \mathrm{i} \lambda^{2} t} \mathrm{~d} \lambda+\int_{0}^{L}(f(\lambda)-f(0)) \mathrm{e}^{-2 \mathrm{i} \lambda^{2} t} \mathrm{~d} \lambda=I_{A}+I_{B} .
$$

The first integral is easily transformed

$$
I_{A}=f(0) \int_{0}^{L} \mathrm{e}^{-2 \mathrm{i} \lambda^{2} t} \mathrm{~d} \lambda=\frac{f(0)}{t^{1 / 2}} \int_{0}^{L t^{1 / 2}} \mathrm{e}^{-2 \mathrm{i} \zeta^{2}} \mathrm{~d} \zeta
$$

and therefore easily uniformly estimated

$$
\left|I_{A}\right| \leqslant \frac{\sqrt{3}|f(0)|}{|t|^{1 / 2}}
$$

In the second integral, one integrates by parts to find

$$
I_{B}=\int_{0}^{L} f^{\prime}(\lambda) \int_{\lambda}^{L} \mathrm{e}^{-2 \mathrm{i} \mu^{2} t} \mathrm{~d} \mu \mathrm{d} \lambda
$$


Therefore,

$$
\begin{aligned}
\left|I_{B}\right| & \leqslant \sup _{0<\lambda<L}\left|\int_{\lambda}^{L} \mathrm{e}^{-2 \mathrm{i} \mu^{2} t} \mathrm{~d} \mu\right| \int_{0}^{L}\left|f^{\prime}(\lambda)\right| \mathrm{d} \lambda \\
& \leqslant\left(\left|\int_{0}^{L} \mathrm{e}^{-2 \mathrm{i} \mu^{2} t} \mathrm{~d} \mu\right|+\sup _{0<\lambda<L}\left|\int_{0}^{\lambda} \mathrm{e}^{-2 \mathrm{i} \mu^{2} t} \mathrm{~d} \mu\right|\right) \int_{0}^{L}\left|f^{\prime}(\lambda)\right| \mathrm{d} \lambda \\
& \leqslant \frac{2 \sqrt{3}}{|t|^{1 / 2}} \int_{0}^{L}\left|f^{\prime}(\lambda)\right| \mathrm{d} \lambda .
\end{aligned}
$$

Combining the estimates for $I_{A}$ and $I_{B}$ establishes the claimed result.

We now want to use these results to estimate $h_{\Delta}(x, y ; t)$. To do this, we want to apply lemmas B.6 or B.7 with $f(\lambda)=\Psi_{\mathrm{d}}^{(\mathrm{e}, \mathrm{o})}(x, 0, \lambda) \Psi_{\mathrm{d}}^{(\mathrm{e}, \mathrm{o})}(y, 0, \lambda)^{*} g_{\Delta}(\lambda)$. Now, from lemma B.2, it is clear that the hypotheses of lemma B.6 concerning the behaviour of $f$ at $\lambda=0$ will only be satisfied in the odd case. Here, we obtain the following.

Lemma B.8. Consider the odd case, and let $\sigma>\frac{5}{2}$. Then $h_{\Delta}(x, y ; t)$ is in $L^{2}\left(\mathbb{R}^{2}\right)$ as a function of $x$ and $y$ with its norm decaying like $|t|^{-3 / 2}$.

Proof. We have the pointwise estimate

$$
\left|h_{\Delta}(x, y ; t)\right| \leqslant \frac{3 \sqrt{3} \Delta\langle x\rangle^{-\sigma}\langle y\rangle^{-\sigma}}{8|t|^{3 / 2}} \sup _{0<\lambda<3 \Delta / 2}\left|\frac{\partial^{2}}{\partial \lambda^{2}}\left(\frac{\Psi_{\mathrm{d}}^{(\mathrm{o})}(x, 0, \lambda) \Psi_{\mathrm{d}}^{(\mathrm{o})}(y, 0, \lambda)^{*} g_{\Delta}(\lambda)}{\lambda}\right)\right| \text {. }
$$

From lemma B.2 we have that the right-hand side is a quadratic polynomial in $|x|$ and $|y|$. Therefore, for $h_{\Delta}(x, y ; t)$ to be in $L^{2}\left(\mathbb{R}^{2}\right)$ as a function of $x$ and $y$ it is sufficient to take $\sigma>\frac{5}{2}$. The time decay of the $L^{2}$ norm is then obvious. Note that each derivative of $g_{\Delta}(\lambda)$ contributes a factor that is $\mathrm{O}\left(\Delta^{-1}\right)$ so the bound on the $L^{2}$ norm scales like $\Delta^{-1}$.

In the even case, we have a zero-energy resonance, and this means that the integrand near $\lambda=0$ is not small enough to allow as rapid decay as in the odd case. In this case, we can only apply lemma B.7 to find the following.

Lemma B.9. Consider the even case, and let $\sigma>\frac{3}{2}$. Then $h_{\Delta}(x, y ; t)$ is in $L^{2}\left(\mathbb{R}^{2}\right)$ as a function of $x$ and $y$, with its norm decaying like $|t|^{-1 / 2}$.

Proof. Using $f(\lambda)=\Psi_{\mathrm{d}}^{(\mathrm{e})}(x, 0, \lambda) \Psi_{\mathrm{d}}^{(\mathrm{e})}(y, 0, \lambda)^{*} g_{\Delta}(\lambda)$ and $L=3 \Delta / 2$ in lemma B.7, we have the pointwise estimate

$$
\left|h_{\Delta}(x, y ; t)\right| \leqslant \frac{\sqrt{3}\langle x\rangle^{-\sigma}\langle y\rangle^{-\sigma}}{|t|^{1 / 2}}\left(|f(0)|+2 \int_{0}^{3 \Delta / 2}\left|f^{\prime}(\lambda)\right| \mathrm{d} \lambda\right) .
$$

Since the derivative with respect to $\lambda$ results in at most linear growth in $x$ and $y$, taking $\sigma>\frac{3}{2}$ is sufficient to ensure that $h_{\Delta}(x, y ; t)$ is in $L^{2}\left(\mathbb{R}^{2}\right)$ as a function of $x$ and $y$. Clearly, for large $t$, the $L^{2}$ norm is $\mathrm{O}\left(|t|^{-1 / 2}\right)$. Note that the estimate is also $\mathrm{O}\left(\Delta^{-1}\right)$ due to differentiation of the bump function $g_{\Delta}(\lambda)$.

In both odd and even cases, the contribution of $h_{\Delta}(x, y ; t)$ to the $L^{2}$ norm of $h(x, y ; t)$ dominates for large time that of $\tilde{h}_{\Delta}(x, y ; t)$, for which we had arbitrary decay. According to lemma B.4, for $\sigma>\frac{5}{2}$ this latter decay is at least as fast as $|t|^{-2}$. These results imply the following. 
Lemma B.10. Fix $\sigma>\frac{5}{2}$. Then, for $t$ sufficiently large, we have the estimates

$$
\left\|\langle\cdot\rangle^{-\sigma}\left(\mathrm{e}^{-\mathrm{i} t \mathcal{B}} \mathcal{P}_{\mathrm{c}}^{(\mathrm{e})} f\right)(\cdot)\right\|_{2} \leqslant \frac{K^{(\mathrm{e})}}{|t|^{1 / 2}}\left\|\langle\cdot\rangle^{\sigma} f(\cdot)\right\|_{2}
$$

and

$$
\left\|\langle\cdot\rangle^{-\sigma}\left(\mathrm{e}^{-\mathrm{i} t \mathcal{B}} \mathcal{P}_{\mathrm{c}}^{(\mathrm{o})} f\right)(\cdot)\right\|_{2} \leqslant \frac{K^{(\mathrm{o})}}{|t|^{3 / 2}}\left\|\langle\cdot\rangle^{\sigma} f(\cdot)\right\|_{2}
$$

where $K^{(\mathrm{e})}$ and $K^{(\mathrm{o})}$ are some positive constants.

This result, taken together with the elementary time-independent bound established in lemma B.1, completes the proof of proposition B.1.

\section{B.2. Singular local decay}

Now we prove the singular local decay estimate for the unitary group $\mathrm{e}^{-\mathrm{i} t \mathcal{B}}$.

Proposition B.2. Let $|\mu| \geqslant \mu_{\min }>0$. Fix $\sigma>\frac{7}{2}$. Let $t=\kappa r$ with $r \geqslant 0$ and $\kappa= \pm 1$. Then, there exist constants $M^{(\mathrm{e}, \mathrm{o})}>0$, such that

$$
\left\|\langle\cdot\rangle^{-\sigma} \lim _{\delta \downarrow 0}\left((\mathcal{B}-2 \mu-2 \mathrm{i} \kappa \delta)^{-1} \mathrm{e}^{-\mathrm{i} t \mathcal{B}} \mathcal{P}_{\mathrm{c}}^{(\mathrm{e})} f\right)(\cdot)\right\|_{2} \leqslant M^{(\mathrm{e})}\langle r\rangle^{-1 / 2}\left\|\langle\cdot\rangle^{\sigma} f(\cdot)\right\|_{2}
$$

and

$\left\|\langle\cdot\rangle^{-\sigma} \lim _{\delta \downarrow 0}\left((\mathcal{B}-2 \mu-2 \mathrm{i} \kappa \delta)^{-1} \mathrm{e}^{-\mathrm{i} t \mathcal{B}} \mathcal{P}_{\mathrm{c}}^{(\mathrm{o})} f\right)(\cdot)\right\|_{2} \leqslant M^{(\mathrm{o})}\langle r\rangle^{-3 / 2}\left\|\langle\cdot\rangle^{\sigma} f(\cdot)\right\|_{2}$.

The constants $M^{(\mathrm{e}, \mathrm{o})}$ depend only on $\mu_{\min }$, so the bounds are uniform for large $|\mu|$.

The proof of this proposition begins with a representation similar to (B.7),

$\langle x\rangle^{-\sigma} \lim _{\delta \downarrow 0}\left((\mathcal{B}-2 \mu-2 \mathrm{i} \kappa \delta)^{-1} \mathrm{e}^{-\mathrm{i} t \mathcal{B}} \mathcal{P}_{\mathrm{c}}^{(\mathrm{e}, \mathrm{o})} f\right)(x)=\int_{-\infty}^{\infty}\langle y\rangle^{\sigma} f(y) k(x, y ; t) \mathrm{d} y$

where

$k(x, y ; t)=\langle x\rangle^{-\sigma}\langle y\rangle^{-\sigma} \lim _{\delta \downarrow 0} \int_{0}^{\infty} \frac{\Psi_{\mathrm{d}}^{(\mathrm{e}, \mathrm{o})}(x, 0, \lambda) \Psi_{\mathrm{d}}^{(\mathrm{e}, \mathrm{o})}(y, 0, \lambda)^{*}}{2 \lambda^{2}-2 \mu-2 \mathrm{i} \kappa \delta} \mathrm{e}^{-2 \mathrm{i} \lambda^{2} t} \mathrm{~d} \lambda$.

Perhaps despite appearances, the kernel $k(x, y ; t)$ is somewhat more amenable to analysis than the kernel $h(x, y ; t)$ that appeared in the non-singular case. This is because for each finite $\delta$ the integrand is absolutely integrable as a consequence of the uniform boundedness in $\lambda$ of $\Psi_{\mathrm{d}}^{(\mathrm{e}, \mathrm{o})}(x, 0, \lambda)$ as guaranteed by lemma B.2 and the large- $\lambda$ behaviour of the denominator.

Again, the goal is to show that the kernel $k(x, y ; t)$ is in $L^{2}\left(\mathbb{R}^{2}\right)$ as a function of $x$ and $y$, with a norm that is decaying in time, although in this case we will only obtain the decay for $t$ of a particular sign. First, we show that the $L^{2}$ norm exists and is finite near $t=0$.

Lemma B.11. Fix $\sigma>\frac{3}{2}, \mu$ with $|\mu| \geqslant \mu_{\min }>0$, and $t$ with $|t|<T$. Then there exist constants $C^{(\mathrm{e}, \mathrm{o})}>0$ depending on $\mu_{\min }$ and $T$ such that

$$
\|k(\cdot, \cdot ; t)\|_{2} \leqslant C^{(\mathrm{e}, \mathrm{o})} .
$$

Since the bounds only depend on $\mu$ via $\mu_{\min }$, they are uniform for large $|\mu|$. 
Proof. Begin by setting $f(\lambda)=\Psi_{\mathrm{d}}^{(\mathrm{e}, \mathrm{o})}(x, 0, \lambda) \Psi_{\mathrm{d}}^{(\mathrm{e}, \mathrm{o})}(y, 0, \lambda)^{*} \mathrm{e}^{-2 \mathrm{i} \lambda^{2} t}$. First we consider $\mu \leqslant-\mu_{\min }<0$, in which case we have

$$
k(x, y ; t)=\frac{\langle x\rangle^{-\sigma}\langle y\rangle^{-\sigma}}{2} \int_{0}^{\infty} \frac{f(\lambda) \mathrm{d} \lambda}{\lambda^{2}-\mu}
$$

since there is no singularity for $\mu<0$. We immediately obtain the pointwise estimate

$$
\begin{aligned}
|k(x, y ; t)| & \leqslant \frac{\langle x\rangle^{-\sigma}\langle y\rangle^{-\sigma}}{2} \sup _{\lambda>0}|f(\lambda)| \int_{0}^{\infty} \frac{\mathrm{d} \lambda}{\lambda^{2}-\mu} \\
& =\frac{\pi\langle x\rangle^{-\sigma}\langle y\rangle^{-\sigma}}{4 \sqrt{-\mu}} \sup _{\lambda>0}|f(\lambda)| \\
& \leqslant \frac{\pi\langle x\rangle^{-\sigma}\langle y\rangle^{-\sigma}}{4 \sqrt{-\mu_{\min }}} \sup _{\lambda>0}|f(\lambda)| .
\end{aligned}
$$

Now we consider $\mu \geqslant \mu_{\min }>0$. Pick some positive $G$ less than $\sqrt{\mu_{\min }}$. Then

$$
k(x, y ; t)=\frac{1}{2}\langle x\rangle^{-\sigma}\langle y\rangle^{-\sigma}\left(I_{\mathrm{ns}}+I_{\mathrm{s}}\right)
$$

where

$$
\begin{aligned}
& I_{\mathrm{ns}} \doteq \int_{0}^{\sqrt{\mu}-G} \frac{f(\lambda) \mathrm{d} \lambda}{\lambda^{2}-\mu}+\int_{\sqrt{\mu}+G}^{\infty} \frac{f(\lambda) \mathrm{d} \lambda}{\lambda^{2}-\mu} \\
& I_{\mathrm{s}} \doteq \lim _{\delta \downarrow 0} \int_{\sqrt{\mu}-G}^{\sqrt{\mu}+G} \frac{f(\lambda) \mathrm{d} \lambda}{(\lambda+\sqrt{\mu+\mathrm{i} \kappa \delta})(\lambda-\sqrt{\mu+\mathrm{i} \kappa \delta})}
\end{aligned}
$$

where the principal branch of the square root is understood, so that the square root is nearly a positive number for $\delta$ small. It is easy to find

$$
\begin{aligned}
\left|I_{\mathrm{ns}}\right| & \leqslant \sup _{\lambda>0}|f(\lambda)|\left(\int_{0}^{\sqrt{\mu}-G} \frac{\mathrm{d} \lambda}{\mu-\lambda^{2}}+\int_{\sqrt{\mu}+G}^{\infty} \frac{\mathrm{d} \lambda}{\lambda^{2}-\mu}\right) \\
& \leqslant \frac{\sup _{\lambda>0}|f(\lambda)|}{\sqrt{\mu}}\left(\operatorname{arctanh}\left(\frac{\sqrt{\mu}}{\sqrt{\mu}+G}\right)+\operatorname{arctanh}\left(\frac{\sqrt{\mu}-G}{\sqrt{\mu}}\right)\right) \\
& \leqslant \sup _{\lambda>0}|f(\lambda)| \sup _{\mu>\mu_{\min }}\left(\frac{1}{\sqrt{\mu}}\left(\operatorname{arctanh}\left(\frac{\sqrt{\mu}}{\sqrt{\mu}+G}\right)+\operatorname{arctanh}\left(\frac{\sqrt{\mu}-G}{\sqrt{\mu}}\right)\right)\right) .
\end{aligned}
$$

For the singular part, we find

$$
\begin{aligned}
I_{\mathrm{S}} & =\lim _{\delta \downarrow 0} \frac{f(\sqrt{\mu})}{\sqrt{\mu}+\sqrt{\mu+\mathrm{i} \kappa \delta}} \int_{\sqrt{\mu}-G}^{\sqrt{\mu}+G} \frac{\mathrm{d} \lambda}{\lambda-\sqrt{\mu+\mathrm{i} \kappa \delta}}+\int_{\sqrt{\mu}-G}^{\sqrt{\mu}+G}\left(\frac{f(\lambda)}{\lambda+\sqrt{\mu}}-\frac{f(\sqrt{\mu})}{2 \sqrt{\mu}}\right) \frac{\mathrm{d} \lambda}{\lambda-\sqrt{\mu}} \\
& =\frac{\mathrm{i} \pi \kappa f(\sqrt{\mu})}{2 \sqrt{\mu}}+\int_{\sqrt{\mu}-G}^{\sqrt{\mu}+G}\left(\frac{f(\lambda)}{\lambda+\sqrt{\mu}}-\frac{f(\sqrt{\mu})}{2 \sqrt{\mu}}\right) \frac{\mathrm{d} \lambda}{\lambda-\sqrt{\mu}} .
\end{aligned}
$$

Therefore,

$$
\begin{aligned}
\left|I_{\mathrm{s}}\right| & \leqslant \frac{\pi|f(\sqrt{\mu})|}{2 \sqrt{\mu}}+2 G \sup _{|\lambda-\sqrt{\mu}|<G}\left|\left(\frac{f(\lambda)}{\lambda+\sqrt{\mu}}-\frac{f(\sqrt{\mu})}{2 \sqrt{\mu}}\right) \frac{1}{\lambda-\sqrt{\mu}}\right| \\
& \leqslant \frac{\pi|f(\sqrt{\mu})|}{2 \sqrt{\mu}}+2 G \sup _{|\lambda-\sqrt{\mu}|<G}\left|\frac{\partial}{\partial \lambda}\left(\frac{f(\lambda)}{\lambda+\sqrt{\mu}}\right)\right| .
\end{aligned}
$$

Again, the bounds are uniform in $\mu$ for large $\mu$. 
Now, we simply note that the pointwise bounds for $I_{\mathrm{ns}}$ and $I_{\mathrm{s}}$ are themselves bounded by functions of $x, y$ and $t$ that grow linearly at worst, as a consequence of differentiation of $f(\lambda)$ with respect to $\lambda$ (cf lemma B.2 for the growth in $x$ and $y$, while the growth in $t$ comes from the factor $\left.\mathrm{e}^{-2 \mathrm{i} \lambda^{2} t}\right)$. Thus, to have $k(x, y ; t) \in L^{2}\left(\mathbb{R}^{2}\right)$ as a function of $x$ and $y$, it is sufficient to take $\sigma>\frac{3}{2}$, and then the norm will be bounded by a linear function of $|t|$, and therefore uniformly for $|t|<T$. The bound is also uniform in $\mu$ for $|\mu| \geqslant \mu_{\min }>0$.

We now note that proving the decay for large $r=|t|$ for $\mu \leqslant-\mu_{\min }<0$ amounts to recalling the non-singular local decay estimate. The integral is not really singular

$$
\begin{aligned}
\lim _{\delta \downarrow 0} \int_{0}^{\infty} \frac{\Psi_{\mathrm{d}}^{(\mathrm{e}, \mathrm{o})}(x, 0, \lambda) \Psi_{\mathrm{d}}^{(\mathrm{e}, \mathrm{o})}(y, 0, \lambda)}{\lambda^{2}-\mu-\mathrm{i} \kappa \delta} \mathrm{e}^{-2 \mathrm{i} \lambda^{2} t} \mathrm{~d} \lambda \\
=\int_{0}^{\infty} \frac{\Psi_{\mathrm{d}}^{(\mathrm{e}, \mathrm{o})}(x, 0, \lambda) \Psi_{\mathrm{d}}^{(\mathrm{e}, \mathrm{o})}(y, 0, \lambda)}{\lambda^{2}-\mu} \mathrm{e}^{-2 \mathrm{i} \lambda^{2} t} \mathrm{~d} \lambda .
\end{aligned}
$$

Using the same arguments as used to prove the non-singular local decay estimate one obtains a pointwise bound for this integral that is at most quadratically growing in $x$ and $y$ and decaying like $|t|^{-1 / 2}$ in the even case and $|t|^{-3 / 2}$ in the odd case. Since the estimates involve up to two derivatives of the quotient in the integrand, the bounds will be uniform in $\mu$ for $\mu \leqslant-\mu_{\min }<0$.

To prove the decay for large $r=|t|$ in the truly singular case when $\mu \geqslant \mu_{\min }>0$, we split $k(x, y ; t)$ into three parts. Let $g_{\Delta}(\lambda)$ and $\tilde{g}_{\Delta}(\lambda)$ be as before, and introduce the new 'bump' functions $g_{G}(\lambda)$ and $\tilde{g}_{G}(\lambda)=1-g_{G}(\lambda)$, both infinitely differentiable and non-negative, with $g_{G}(\lambda)$ identically equal to zero outside of the interval $(\sqrt{\mu}-3 G / 2, \sqrt{\mu}+3 G / 2)$ and identically equal to one inside of the interval $(\sqrt{\mu}-G / 2, \sqrt{\mu}+G / 2)$. Set

$$
k(x, y ; t)=\frac{1}{2}\langle x\rangle^{-\sigma}\langle y\rangle^{-\sigma}\left(I_{0}+I_{\mu}+\tilde{I}\right)
$$

where

$$
\begin{aligned}
& I_{0} \doteq \int_{0}^{3 \Delta / 2} \frac{\Psi_{\mathrm{d}}^{(\mathrm{e}, \mathrm{o})}(x, 0, \lambda) \Psi_{\mathrm{d}}^{(\mathrm{e}, \mathrm{o})}(y, 0, \lambda)^{*}}{\lambda^{2}-\mu} g_{\Delta}(\lambda) \mathrm{e}^{-2 \mathrm{i} \lambda^{2} t} \mathrm{~d} \lambda \\
& I_{\mu} \doteq \lim _{\delta \downarrow 0} \int_{\sqrt{\mu}-3 G / 2}^{\sqrt{\mu}+3 G / 2} \frac{\Psi_{\mathrm{d}}^{(\mathrm{e}, \mathrm{o})}(x, 0, \lambda) \Psi_{\mathrm{d}}^{(\mathrm{e}, \mathrm{o})}(y, 0, \lambda)^{*}}{\lambda^{2}-\mu-\mathrm{i} \kappa \delta} g_{G}(\lambda) \mathrm{e}^{-2 \mathrm{i} \lambda^{2} t} \mathrm{~d} \lambda
\end{aligned}
$$

and

$$
\begin{aligned}
\tilde{I} \doteq \int_{\Delta / 2}^{\sqrt{\mu}-G / 2} & \frac{\Psi_{\mathrm{d}}^{(\mathrm{e}, \mathrm{o})}(x, 0, \lambda) \Psi_{\mathrm{d}}^{(\mathrm{e}, \mathrm{o})}(y, 0, \lambda)^{*}}{\lambda^{2}-\mu} \tilde{g}_{\Delta}(\lambda) \tilde{g}_{G}(\lambda) \mathrm{e}^{-2 \mathrm{i} \lambda^{2} t} \mathrm{~d} \lambda \\
& +\int_{\sqrt{\mu}+G / 2}^{\infty} \frac{\Psi_{\mathrm{d}}^{(\mathrm{e}, \mathrm{o})}(x, 0, \lambda) \Psi_{\mathrm{d}}^{(\mathrm{e}, \mathrm{o})}(y, 0, \lambda)^{*}}{\lambda^{2}-\mu} \tilde{g}_{G}(\lambda) \mathrm{e}^{-2 \mathrm{i} \lambda^{2} t} \mathrm{~d} \lambda .
\end{aligned}
$$

Note that in keeping the contributions near zero and near $\mu$ distinct, we are assuming that $3 \Delta / 2<\sqrt{\mu_{\min }}-G$. The analysis of $I_{0}$ and $\tilde{I}$ proceeds exactly as in the proof of the nonsingular local decay estimate. The results are almost identical. For $\tilde{I}$ one can integrate by parts as many times as one likes, and therefore one obtains a pointwise estimate with arbitrary decay in time of order $\mathrm{O}\left(|t|^{-k}\right)$ for $k \geqslant 2$, but at the cost of polynomial growth in $x$ and $y$ of degree $k$. For $I_{0}$, one obtains a pointwise estimate that decays like $\mathrm{O}\left(|t|^{-1 / 2}\right)$ in the even case and $\mathrm{O}\left(|t|^{-3 / 2}\right)$ in the odd case, at the cost of quadratic growth in $x$ and $y$.

The estimates of $I_{0}$ and $\tilde{I}$ are uniform for large $\mu$. The pointwise bounds for $\tilde{I}$ involve supremum bounds over the range of integration of the quantity

$$
\lambda^{2}\left(\mathcal{A}^{k} \frac{f(\cdot)}{(\cdot)^{2}-\mu}\right)(\lambda)
$$


with $f(\lambda)$ given by $\Psi_{\mathrm{d}}^{(\mathrm{e}, \mathrm{o})}(x, 0, \lambda) \Psi_{\mathrm{d}}^{(\mathrm{e}, \mathrm{o})}(y, 0, \lambda)^{*} \tilde{g}_{G}(\lambda)$ for $\lambda>\sqrt{\mu}+G / 2$ and with $f(\lambda)$ given by $\Psi_{\mathrm{d}}^{(\mathrm{e}, \mathrm{o})}(x, 0, \lambda) \Psi_{\mathrm{d}}^{(\mathrm{e}, \mathrm{o})}(y, 0, \lambda)^{*} \tilde{g}_{\Delta}(\lambda) \tilde{g}_{G}(\lambda)$ for $\Delta / 2<\lambda<\sqrt{\mu}-G / 2$. In particular, we will need these bounds for $k=2$, in which case

$$
\lambda^{2}\left(\mathcal{A}^{k} \frac{f(\cdot)}{(\cdot)^{2}-\mu}\right)(\lambda)=\left(\frac{15 \lambda^{4}-10 \mu \lambda^{2}+3 \mu}{\lambda^{2}\left(\lambda^{2}-\mu\right)^{3}}\right) f(\lambda)+\left(\frac{-7 \lambda^{2}+3 \mu}{\lambda\left(\lambda^{2}-\mu\right)^{2}}\right) f^{\prime}(\lambda)+\frac{f^{\prime \prime}(\lambda)}{\lambda^{2}-\mu} .
$$

For the part of $\tilde{I}$ involving $\lambda>\sqrt{\mu}+G / 2$, it is easy to check that the three coefficients above are monotonic functions of $\lambda$ for $\lambda>\sqrt{\mu}$ that decay for large $\lambda$ with $\mu$ fixed. Therefore, each coefficient is bounded by its magnitude at the lower endpoint $\lambda=\sqrt{\mu}+G / 2$. With $G$ held fixed, these bounds are then seen to be decaying functions of $\mu$. For the part of $\tilde{I}$ involving $\lambda \in(\Delta / 2, \sqrt{\mu}-G / 2)$, it is easy to see that the coefficients blow up at both endpoints. Therefore, for $\Delta$ and $G$ sufficiently small but independent of $\mu$, the coefficients will be bounded by the maximum of their values at $\lambda=\Delta / 2$ and $\lambda=\sqrt{\mu}-G / 2$. Again, holding $\Delta$ and $G$ fixed, one sees that the bounds are uniform for large $\mu$. This direct argument shows that, at least for $k=2$, the pointwise bound for $\tilde{I}$ is uniform in $\mu$. Establishing the uniformity of the pointwise estimate for $I_{0}$ is easier; the denominator $\lambda^{2}-\mu$ plays no essential role for $\lambda<3 \Delta / 2$ for $\mu$ sufficiently large.

The new term that must be handled differently is $I_{\mu}$.

Lemma B.12. For all $k \geqslant 2$, the integral $I_{\mu}$ satisfies the pointwise estimate

$$
\left|I_{\mu}\right| \leqslant \frac{2}{k-1} \frac{P_{k}(x, y)}{|t|^{k-1}}
$$

where $P_{k}(x, y)$ is a polynomial in $|x|$ and $|y|$ of degree $k$ with positive coefficients that are uniform in $\mu$.

Proof. Consider first $t>0$. Then, the quantity to estimate is

$$
\begin{aligned}
I_{\mu} & =\lim _{\delta \downarrow 0} \int_{\sqrt{\mu}-3 G / 2}^{\sqrt{\mu}+3 G / 2} \frac{\Psi_{\mathrm{d}}^{(\mathrm{e}, \mathrm{o})}(x, 0, \lambda) \Psi_{\mathrm{d}}^{(\mathrm{e}, \mathrm{o})}(y, 0, \lambda)^{*}}{\lambda^{2}-\mu-\mathrm{i} \delta} g_{G}(\lambda) \mathrm{e}^{-2 \mathrm{i} \lambda^{2} t} \mathrm{~d} \lambda \\
& =2 \mathrm{ie}^{-2 \mathrm{i} \mu t} \lim _{\delta \downarrow 0} \mathrm{e}^{2 \delta t} \int_{\sqrt{\mu}-3 G / 2}^{\sqrt{\mu}+3 G / 2} \Psi_{\mathrm{d}}^{(\mathrm{e}, \mathrm{o})}(x, 0, \lambda) \Psi_{\mathrm{d}}^{(\mathrm{e}, \mathrm{o})}(y, 0, \lambda)^{*} g_{G}(\lambda) \int_{t}^{\infty} \mathrm{e}^{-2 \mathrm{i}\left(\lambda^{2}-\mu-\mathrm{i} \delta\right) s} \mathrm{~d} s \mathrm{~d} \lambda \\
& =2 \mathrm{ie}^{-2 \mathrm{i} \mu t} \int_{t}^{\infty} \mathrm{e}^{2 \mathrm{i} \mu s}\left[\int_{\sqrt{\mu}-3 G / 2}^{\sqrt{\mu}+3 G / 2} \Psi_{\mathrm{d}}^{(\mathrm{e}, \mathrm{o})}(x, 0, \lambda) \Psi_{\mathrm{d}}^{(\mathrm{e}, \mathrm{o})}(y, 0, \lambda)^{*} g_{G}(\lambda) \mathrm{e}^{-2 \mathrm{i} \lambda^{2} s} \mathrm{~d} \lambda\right] \mathrm{d} s .
\end{aligned}
$$

Now, with $g_{G}(\lambda)$ vanishing to all orders at the integration endpoints, it is possible to bound the integral in square brackets by iterated integration by parts. The bound is $\mathrm{O}\left(|s|^{-k}\right)$ and grows in $x$ and $y$ like a polynomial $P_{k}(x, y)$ of degree $k$. This bound is uniform in $\mu$, since the only place $\mu$ appears is in the range of integration over which bounds are required, and from lemma B. 2 we know that these bounds are uniform for all $\lambda$. Therefore, we have

$$
\left|I_{\mu}\right| \leqslant 2 \int_{t}^{\infty} P_{k}(x, y)|s|^{-k} \mathrm{~d} s=\frac{2}{k-1} \frac{P_{k}(x, y)}{|t|^{k-1}}
$$

which establishes the lemma for $t>0$. For $t<0$, one obtains an integral from $-\infty$ to $t$ in the second step above, and ultimately obtains the same bound.

Finally, we put the pieces together to complete the proof of proposition B.2. For the odd case, we want decay of order $\mathrm{O}\left(|t|^{-3 / 2}\right)$. For $\langle x\rangle^{-\sigma}\langle y\rangle^{-\sigma} I_{0}$ to be in $L^{2}\left(\mathbb{R}^{2}\right)$ with this decay 
rate, we need $\sigma>\frac{5}{2}$. With this bound on $\sigma$, we can obtain $\langle x\rangle^{-\sigma}\langle y\rangle^{-\sigma} \tilde{I}$ to be in $L^{2}\left(\mathbb{R}^{2}\right)$ with decay bounded by $\mathrm{O}\left(|t|^{-2}\right)=\mathrm{o}\left(|t|^{-3 / 2}\right)$, but no better. Finally, for $\langle x\rangle^{-\sigma}\langle y\rangle^{-\sigma} I_{\mu}$ being in $L^{2}\left(\mathbb{R}^{2}\right)$ with decay $\mathrm{O}\left(|t|^{-2}\right)$ we now see that we need to localize a bit more in space by taking $\sigma>\frac{7}{2}$. Combining these large time estimates with the finite-time bound of lemma B.11 establishes the proposition in the odd case. Similar arguments for the even case give an $L^{2}\left(\mathbb{R}^{2}\right)$ norm that decays like $\mathrm{O}\left(|t|^{-1 / 2}\right)$ for $\sigma>\frac{7}{2}$.

Remark. Evidently, the singular decay estimates blow up when $\mu_{\min }$ approaches zero. This is an essential phenomenon in both the odd and even cases. This is best seen by considering the singular integral for the case $\mu=0$

$$
\int_{0}^{\infty} \frac{\Psi_{\mathrm{d}}^{(\mathrm{e}, \mathrm{o})}(x, 0, \lambda) \Psi_{\mathrm{d}}^{(\mathrm{e}, \mathrm{o})}(y, 0, \lambda)^{*}}{\lambda^{2}-\mathrm{i} \kappa \delta} \mathrm{e}^{-2 \mathrm{i} \lambda^{2} t} \mathrm{~d} \lambda .
$$

This integral blows up for all $x, y$ and $t$, as $\delta$ tends to zero in the even case. In the odd case there is sufficient vanishing at $\lambda=0$ for the limit of $\delta \downarrow 0$ to exist for all $x, y$ and $t$, but the limit only decays in $t$ like $|t|^{-1 / 2}$. Thus it is not possible for estimates of the form derived for $|\mu| \geqslant \mu_{\min }>0$ to hold uniformly in any neighbourhood of $\mu=0$.

\section{References}

[1] Arnold V I 1980 Ordinary Differential Equations (Cambridge, MA: MIT Press)

[2] Besley J A, Akhmediev N N and Miller P D 1997 Modes of periodic waveguides Opt. Lett. $221162-4$

[3] Besley J A, Akhmediev N N and Miller P D 1998 Periodic optical waveguides: exact Floquet theory and spectral properties Stud. Appl. Math. $101343-55$

[4] Birnir B 1994 Qualitative analysis of radiating breathers Commun. Pure Appl. Math. 47 103-19

[5] Birnir B, McKean H P and Weinstein A 1994 The rigidity of sine-Gordon breathers Commun. Pure Appl. Math. 47 1043-51

[6] Cycon H L, Froese R G, Kirsch W and Simon B 1987 Schrödinger Operators with Applications to Quantum Mechanics and Global Geometry (Berlin: Springer)

[7] Costin O, Lebowitz J L and Rokhlenko A 1999 Ionization of a model atom: exact results and connection with experiment Phys. Rev. Lett. submitted

[8] Denzler J 1993 Nonpersistence of breather families for the perturbed sine-Gordon equation Commun. Math. Phys. 158 397-430

[9] Faddeev L D and Takhtajan L A 1987 Hamiltonian Methods in the Theory of Solitons (Berlin: Springer)

[10] Galindo A and Pascal P 1991 Quantum Mechanics vol II (Berlin: Springer)

[11] Hislop P D and Sigal I M 1996 Introduction to Spectral Theory: with Applications to Schrödinger Operators (Berlin: Springer)

[12] Kevorkian J and Cole J D 1981 Perturbation Methods in Applied Mathematics (Berlin: Springer)

[13] Kevrekides P G and Weinstein M I 1999 Dynamics of lattice kinks Physica D submitted

[14] Kichenassamy S 1991 Breather solutions of nonlinear wave equations Commun. Pure Appl. Math. 44789

[15] Kirr E and Weinstein M I 1999 Parametrically excited Hamiltonian partial differential equations SIAM J. Math. Anal. submitted

[16] Kirr E and Weinstein M I 2000 in preparation

[17] Landau L D and Lifshitz E M 1965 Quantum Mechanics, Non-relativistic Theory (Course in Theoretical Physics vol 3) (Oxford: Pergamon)

[18] MacKay R S and Aubry S 1994 Proof of existence of breathers for time-reversible or Hamiltonian networks of weakly coupled oscillators Nonlinearity 7 1623-43

[19] Manakov S V 1974 On the theory of two-dimensional stationary self-focusing of electromagnetic waves Sov. Phys.-JETP 38 248-53

[20] Marcuse D 1974 Theory of Dielectric Optical Waveguides (New York: Academic)

[21] Miller P D and Akhmediev N N 1996 Transfer matrices for multiport devices made from solitons Phys. Rev. E 53 4098-106

[22] Miller P D and Akhmediev N N 1998 Modal expansions and completeness relations for some time-dependent Schrödinger equations Physica D 123 513-24 
[23] Miller P D, Soffer A and Weinstein M I 2000 in preparation

[24] Newell A C and Moloney J V 1992 Nonlinear Optics (Redwood City, CA: Addison-Wesley)

[25] Sigal I M 1993 Nonlinear wave and Schrödinger equations. I. Instability of periodic and quasiperiodic solutions Commun. Math. Phys. 153 297-320

[26] Soffer A and Weinstein M I 1998 Time dependent resonance theory Geom. Funct. Anal. 8 1086-128

[27] Soffer A and Weinstein M I 1999 Resonances, radiation damping and instability in Hamiltonian nonliear wave equations Inventiones Math. 136 9-74

[28] Soffer A and Weinstein M I 1998 Nonautonomous Hamiltonians J. Statist. Phys. 93 359-91 